\title{
Standardsetzung als rekursiver Prozess
}

\subsection{Normermittlung unter Zuhilfenahme der Deduktion und Induktion als Erklärungsansätze}

\subsubsection{Deduktive Normermittlung}

\subsubsection{Normativer Charakter von Rechnungslegungsvorschriften}

Zur Beurteilung des Einflusses der Rechnungslegungspraxis auf die Entstehung von Rechnungslegungsvorschriften bedarf es zunächst einer Auseinandersetzung mit der Rechtsnatur von Bilanzierungsnormen sowie der Frage nach der Normermittlung. Rechtsnormen umfassen neben den im Rahmen eines Gesetzgebungsverfahrens kodifizierten Normen auch Richter- und Gewohnheitsrecht. ${ }^{1}$ Während als Richterrecht die in ständiger Rechtsprechung entwickelten Normen gelten, sind die durch dauerhafte und allgemeine Anwendung der im Rechtsverkehr verbindlich anerkannten Normen unter dem Gewohnheitsrecht zu subsumieren. $^{2}$ Von Rechtsnormen zu unterscheiden sind hingegen fachtechnische Normen, die von - meist privatrechtlichen - Sachverständigengremien bzw. Standardisierungsausschüssen entwickelt werden. ${ }^{3}$ Derartige Fachnormen entfalten lediglich freiwilligen Anwendungscharakter, sofern sie nicht durch den Gesetzgeber oder Gerichte anerkannt werden. ${ }^{4}$ Die Qualifikation von Rechnungslegungsvorschriften als Rechts- oder Fachnormen bestimmt sich folglich nach

\footnotetext{
${ }^{1}$ Vgl. Preißler (2005), S. 25-27; zum Rechtsnormcharakter von Rechnungslegungsnormen Döllerer (1959), S. 1217 f.; Beisse (1990), S. 499 f.; Berndt (2005), S. 78.

${ }^{2}$ Vgl. Vogel (1998), S. 39, 83-91.

${ }^{3}$ Vgl. zum Fachnormcharakter von Rechnungslegungsvorschriften Biener (1996), S. 62-66; Botzem/Hofmann (2009), S. 231-234.

${ }^{4}$ Vgl. Wüstemann (1999), S. 95.

(C) Der/die Autor(en) 2022

A. Jendreck, Die Rolle von Unternehmen im IFRS-Standardsetzungsprozess,

Rechnungswesen und Unternehmensüberwachung,

https://doi.org/10.1007/978-3-658-36129-7_2
} 
der jeweiligen Rechtsquelle. ${ }^{5}$ Im Rahmen der Normermittlung werden einzelne Rechnungslegungsvorschriften entsprechend vom Gesetzgeber, der Rechtsprechung, dem Rechtsverkehr und/oder einem Fachgremium konkretisiert. ${ }^{6}$ „Die Konkretisierung besteht darin, daß die Normen im Hinblick auf die Eigenart und Erfordernisse der zu beurteilenden Fallgestaltungen anwendbar gemacht werden. " 7 Rechnungslegungsvorschriften weisen somit normativen Charakter auf, da die Festlegung des Regelungsinhalts immer ein vom Normsetzer vorzunehmendes Werturteil verlangt. ${ }^{8}$ Im Standardsetzungsprozess der IFRS obliegt diese Wertungskompetenz grundsätzlich dem IASB. ${ }^{9}$ Zwar erlangen die IFRS aufgrund des Endorsement-Prozesses innerhalb der EU Rechtsnormcharakter und können somit de jure vom europäischen Gesetzgeber sowie letztlich durch den Europäischen Gerichtshof (EuGH) konkretisiert werden. ${ }^{10}$ Materiell werden sie jedoch vom IASB erstellt ${ }^{11}$ und fast gänzlich ohne Änderungen im Endorsement-Prozess übernommen, weshalb sie originär als Fachnormen einzustufen sind ${ }^{12}$. Inwieweit das IASB der ihm übertragenen normativen Aufgabe im Standardsetzungsprozess nachkommt, wird dabei wesentlich von der Art der Normermittlung beeinflusst.

\subsubsection{Deduktion systemkohärenter Bilanzierungsnormen}

\subsection{Ableitung von Rechnungslegungsvorschriften aus übergeordneten Rechnungslegungszwecken}

Die Normermittlung kann deduktiv oder induktiv erfolgen: ${ }^{13}$ Während der induktive Ansatz ${ }^{14}$ auf die Generalisierbarkeit eines Ergebnisses auf Basis von Einzeltatsachen - häufig empirischer Beobachtungen - abzielt, ist unter dem deduktiven Ansatz die Ableitung einzelner, spezieller sich aus allgemeingültigen

${ }^{5}$ Vgl. Kruse (1978), S. 17; zur Diskussion der Rechtsquellenqualität der IFRS Müller (2015), S. 747-757.

${ }^{6}$ Vgl. Biener (1996), S. 59-66.

${ }^{7}$ Beisse (1990), S. 502 f.

${ }^{8}$ Vgl. Moxter (1976), S. 90; Wüstemann/Kierzek (2007b), S. 361; Fülbier u. a. (2009), S. 460; Schmitz (2012), S. 152.

${ }^{9} \mathrm{Vgl}$. Kirchhof (2008), S. 176 f.

${ }^{10}$ Vgl. IAS-Verordnung, Artikel 3; Schön (2004), S. 764; Wojcik (2008), S. 252; Najderek (2009), S. $98 \mathrm{f}$.

11 Vgl. Euler (2002), S. 876.

${ }^{12}$ Vgl. Najderek (2009), S. 99.

13 Vgl. Wüstemann (2002), S. 99-119.

${ }^{14} \mathrm{Vgl}$. Abschnitt 2.1.2. 
Fakten, Theorien oder Annahmen ergebender Schlussfolgerungen zu verstehen. ${ }^{15}$ Sofern als Deduktionsbasis ein bestimmtes Ziel als Annahme zugrunde gelegt wird, handelt es sich um einen normativen Ansatz, da der festgelegte Zweck selbst eine Wertprämisse darstellt. ${ }^{16}$ Rechnungslegungsvorschriften werden unter einem deduktiven Ansatz demzufolge durch "Nachdenken“ über sachgerechte Bilanzierungslösungen ermittelt, die anhand der gesetzten Rechnungslegungszwecke abzuleiten sind. ${ }^{17}$ Der Rechnungslegungszweck selbst muss wiederum jedoch „,von außen normativ vorgegeben werden“. ${ }^{18}$ Im Rahmen der Normermittlung erfolgt dann eine den Rechnungslegungszweck konkretisierende Normierung allgemeiner Grundsätze und Rechnungslegungsprinzipien bzw. qualitativer Anforderungen, etwa der Objektivierung und wirtschaftlichen Betrachtungsweise, ${ }^{19}$ der Relevanz und der glaubwürdigen Darstellung, ${ }^{20}$ sowie der ihnen folgenden Einzelvorschriften, ${ }^{21}$ die sich zu „einem kohärenten Ganzen fügen““22. Ein derartiges zweckkohärentes, auf allgemeinen Prinzipien basierendes Rechnungslegungssystem erfordert nicht notwendigerweise umfangreiche Einzelnormen, da Bilanzierungssachverhalte durch Abwägung allgemeiner Rechnungslegungsprinzipien konkretisiert werden können. ${ }^{23}$ Das IASB strebt eine deduktive Standardsetzung mithilfe des Rahmenkonzepts an, das im Gegensatz zu einer rein prinzipienbasierten Rechnungslegung eine subsidiäre Funktion gegenüber den Einzelstandards einnimmt. ${ }^{24}$

\subsection{Rahmenkonzept als Grundlage deduktiver Standardsetzung}

\subsection{Entwicklung eines Rahmenkonzepts für die Standardsetzung}

Die Bedeutung eines Rahmenkonzepts für die internationale Rechnungslegung hat ihren Ursprung in der bilanztheoretischen Forschung, die sich bei der

${ }^{15}$ Vgl. Ijiri (1975), S. 5 f.

${ }^{16}$ Vgl. Ijiri (1975), S. 6; Schmitz (2012), S. 151-154.

17 Vgl. Döllerer (1959), S. 1220 (auch Zitat); zur Abgrenzung der formallogischen Ableitung von der Deduktion aus dem Normsystem Beisse (1994), S. 13 f., 19 f.

${ }^{18}$ Vgl. Schmitz (2012), S. 154 (auch Zitat).

${ }^{19}$ Vgl. grundlegend Euler (1997), S. 181 f.; Moxter (2003), S. 15-17.

${ }^{20}$ Vgl. Christensen (2010), S. 293 f.

${ }^{21}$ Vgl. Moxter (1976), S. 90 f.; Hendriksen/van Breda (1992), S. 15 f.; Berndt (2005), S. 79; Wüstemann/Kierzek (2007b), S. 360-362.

${ }^{22}$ Berndt (2005), S. 82.

${ }^{23}$ Vgl. Beisse (1997), S. 403; Euler (1997), S. 184 f.

${ }^{24}$ Vgl. Preface (2019), Rz. 3 (a); zur Unterscheidung zwischen prinzipien- und regelbasierten Rechnungslegungsstandards Schipper (2003), S. 62-71; Preißler (2005), S. 17-22; Benston u. a. (2006), S. 167-171. 
Formulierung von Rechnungslegungsprinzipien von einer anfänglich deskriptiven Aufgabe zu einem normativen Ansatz entwickelte. ${ }^{25}$ Im Zuge der USamerikanischen Rechnungslegungsregulierung, die in ihren Grundzügen durch eine deskriptive Kodifizierung der allgemeinen Bilanzierungspraxis geprägt war, erfolgte dieser normativ-theoretische Wandel durch die jahrelange Entwicklung und Überarbeitung einer konzeptionellen Grundlage, die 1973 mit dem sog. Trueblood-Committee-Report in Form grundlegender Rechnungslegungsziele 26 und qualitativer Anforderungen normiert wurde. ${ }^{27}$ Das zeitgleich gegründete FASB nutzte dies als Ausgangsbasis für die Entwicklung eines Rahmenkonzepts, das die Rechnungslegungsziele und qualitativen Anforderungen sowie Definitions-, Ansatz- und Bewertungsprinzipien von Bilanzposten konkretisierte und damit einen normativen Maßstab für die künftige Standardsetzung festlegte. ${ }^{28}$ Obwohl das International Accounting Standards Committee (IASC) als Vorgängerorganisation des IASB zur gleichen Zeit gegründet wurde, erfolgte die Entwicklung der International Accounting Standards (IAS) in einer ersten deskriptiven Phase zunächst ohne konzeptionelle Basis durch Zusammentragen und Bearbeiten verschiedener nationaler Bilanzierungspraktiken. ${ }^{29} \mathrm{Um}$ die Unschärfen und zahlreichen Wahlrechte dieser ,,additiv entwickelten Standards“30 zu reduzieren und die Vergleichbarkeit der auf Grundlage von IAS erstellten Jahresabschlüsse zu erhöhen, wurden die einzelnen Standards überarbeitet und inhaltlich präzisiert. ${ }^{31}$ Im Zuge dessen wurde 1989 das erste Rahmenkonzept veröffentlicht, ${ }^{32}$ das sich im Wesentlichen an den Inhalten des US-amerikanischen Rahmenkonzepts orientierte und die normative Grundlage für die weitere Standardsetzung bildete. ${ }^{33}$

\footnotetext{
25 Vgl. Chatfield (1977), S. 232-250; für einen detaillierten Überblick zur Entwicklung der Bilanzrechtsforschung Schmitz (2012), S. 9-126; Previts/Flesher (2015), S. 39-55.

${ }^{26}$ Vgl. zur Entwicklung und Bedeutung des Trueblood-Committees für die Ausrichtung auf die Entscheidungsnützlichkeit insgesamt Zeff (2016).

${ }^{27}$ Vgl. insgesamt AICPA (1973); Zeff (1999), S. 90-101; Foster/Johnson (2001), S. 2 f.

${ }^{28}$ Vgl. Gore (1992), S. 33 f.; Storey (2003), S. 33-64; Zeff (1999), S. 101-119; Foster/Johnson (2001), S. 1.

${ }^{29}$ Vgl. Thorell/Whittington (1994), S. 224; zum Überblick der ersten Standards Camfferman/Zeff (2007), S. 90-143.

${ }^{30}$ Botzem (2010), S. 81.

${ }^{31}$ Vgl. Thorell/Whittington (1994), S. 224; Camfferman/Zeff (2007), S. 264-286.

32 Vgl. Framework (1989), Vorwort.

${ }^{33}$ Vgl. Gore (1992), S. 126 f.; Ballwieser (2014), S. 453.
} 
Zur Verbesserung und Klarstellung bestehender sowie Angleichung nicht übereinstimmender Prinzipien begannen das IASB und FASB im Oktober 2004 mit der Entwicklung eines gemeinsamen Rahmenkonzepts. ${ }^{34}$ Die Zusammenarbeit endete jedoch 2010 mit der Veröffentlichung der gemeinsam überarbeiteten Ziel- und Zwecksetzung sowie qualitativen Anforderungen. ${ }^{35}$ Aufgrund der zwischen den Boards divergierenden Ansichten zu den Definitions-, Ansatz- und Bewertungs- sowie Darstellungs- und Offenlegungsprinzipien arbeiteten beide Standardsetzer getrennt voneinander an den weiteren Rahmenkonzeptkapiteln. ${ }^{36}$ Die eigenständige Weiterentwicklung führte teilweise jedoch auch zu Änderungen an den 2010 veröffentlichen Zwecken und qualitativen Anforderungen, ${ }^{37}$ wodurch die ursprünglich angestrebte Konvergenz zwischen dem vom IASB in 2018 veröffentlichten finalen Rahmenkonzept und dem bislang noch nicht finalisierten US-amerikanischen Rahmenkonzept voraussichtlich nur begrenzt erreicht wird.

\subsection{Bedeutung des Rahmenkonzepts für die IFRS-Standardsetzung}

Trotz der zum Teil unterschiedlichen Ausgestaltung einzelner Rahmenkonzeptprinzipien ist in beiden Rahmenkonzepten dessen normative Funktion für die Standardsetzung explizit verankert. ${ }^{38}$ Zwar ist das Rahmenkonzept selbst kein IFRS-Standard und somit bei der Bilanzierung von Geschäftsvorfällen den Einzelstandards nachgelagert, ${ }^{39}$ es erlangt über den nach IAS 1.15 erforderlichen Einbezug bei der Ermittlung eines den tatsächlichen Verhältnissen entsprechenden Bilds und der nach IAS 8.11 geforderten Berücksichtigung bei Regelungslücken jedoch auch bei der Standardanwendung Gültigkeit. ${ }^{40}$ Als Rechnungslegungszweck und Grundprinzipien festlegender Maßstab soll es dem Standardsetzer demnach bei der Entwicklung und Überarbeitung von Regelungsvorschriften auf Einzelstandardebene dienen sowie von Anwendern und anderen Adressaten als Interpretationshilfe und zur Schließung von Regelungslücken herangezogen werden. ${ }^{41}$ Das Rahmenkonzept fungiert daher als Deduktionsbasis für die Ermittlung zweckadäquater Normen, um konsistente und nachvollziehbare Standards

\footnotetext{
${ }^{34}$ Vgl. IASB (2004), S. 4; für einen detaillierten Überblick über das gemeinsame Standardsetzungsprojekt Pelger (2016), S. 55-67.

${ }^{35} \mathrm{Vgl}$. CF (2018), BC0.3.

36 Vgl. Erb/Pelger (2013), S. 518; Ballwieser (2014), S. 453 f.; Herz (2014), S. 101.

${ }^{37} \mathrm{Vgl} . \mathrm{CF}$ (2018), BC.0.9.

${ }^{38}$ Vgl. CF (2018), SP1.1 i. V. m. 1.11; FASB (2010), Einleitung.

${ }^{39} \mathrm{Vgl}$. CF (2018), SP1.2.

40 Vgl. Pelger (2012), S. 65; Hoffmann/Detzen (2012), S. 54; Ruhnke/Nerlich (2004), S. 392.

${ }^{41}$ Vgl. CF (2018), SP1.1.
} 
sicherzustellen. ${ }^{42}$ Außerdem ermöglicht es dem IASB und FASB im Rahmen der Standardsetzung ,standhaft und nachprüfbar eine Position [zu] vertreten“43 und die vorgenommene Normkonkretisierung auf Einzelstandardebene sowie die dabei getroffenen Wertungsentscheidungen zu rechtfertigen. ${ }^{44}$

Die Bedeutung des Rahmenkonzepts für eine dem deduktiven Ansatz entsprechende konsistente Standardsetzung wird indes durch die jahrelange Rahmenkonzeptüberarbeitung bei gleichzeitiger Entwicklung neuer IFRS-Standards konterkariert. ${ }^{45}$ Zwar erfolgte die Entwicklung neuer Standards unter Antizipation der neuen Rahmenkonzeptregelungen, jedoch war die formale Grundlage weiterhin das alte Rahmenkonzept, was zu terminologischen und konzeptionellen Unschärfen führte. Diese Inkonsistenzen wurden besonders bei den Konvergenzprojekten mit dem FASB, wie der Entwicklung von IFRS 15 und IFRS 16, aufgrund bestehender Abweichungen zwischen den jeweils alten Rahmenkonzepten noch verstärkt. Bspw. ist die Anforderung „reasonable“ an zahlreichen Stellen in IFRS 15 normiert ${ }^{46}$ und findet sich auch im Rahmenkonzept vereinzelt wie$\operatorname{der}^{47}$, es stellt jedoch weder eine qualitative Anforderung dar, noch erfolgt eine Konkretisierung des Begriffs.

Zudem bestehen aufgrund der Rahmenkonzeptüberarbeitung Inkonsistenzen zwischen den alten IFRS-Standards und dem neuen Rahmenkonzept. So knüpfen verschiedene IFRS-Standards an die Prinzipien des alten Rahmenkonzepts an, wie bspw. die Anforderung der Verlässlichkeit als Ansatzkriterium in IAS 16.7 oder IAS 38.21 oder zur Schließung von Regelungslücken in IAS 8.10, ohne dass eine Anpassung an die veränderten Rahmenkonzeptprinzipien vorgenommen wurde. ${ }^{48}$ Auch die Regelungen des IAS 37 Rückstellungen, Eventualverbindlichkeiten und Eventualforderungen, für die zunächst eine Überarbeitung begonnen, letztlich jedoch unter Antizipation der Rahmenkonzeptänderungen abgebrochen wurde, ${ }^{49}$ weisen Inkonsistenzen zum neuen Rahmenkonzept auf. ${ }^{50}$ Da die Überarbeitung

\footnotetext{
${ }^{42}$ Vgl. Ballwieser (2014), S. 452.

43 Ballwieser (2005), S. 745.

${ }^{44}$ Vgl. zum Rahmenkonzept als Legitimationsbasis Hines (1989), S. 85-88; zur Charakterisierung des Rahmenkonzepts Dennis (2019), S. 2-12.

${ }^{45}$ Vgl. McGregor/Street (2007), S. 45.

$46 \mathrm{Vgl}$. etwa IFRS 15.44, IFRS 15.54, IFRS 15.67, IFRS 15.78.

${ }^{47}$ Vgl. CF (2018), 2.19, CF (2018), 2.36, CF (2018), 5.19.

48 Vgl. zur Problematik des IAS 8.10 Hoffmann/Detzen (2012), S. 54.

${ }^{49} \mathrm{Vgl}$. Morley (2016), S. $226 \mathrm{f}$.

${ }^{50}$ Vgl. Erb/Pelger (2018a), S. 329.
} 
dieser Standards vorerst weiterhin aussteht, scheint die angestrebte Funktion des Rahmenkonzepts als eine die Einzelstandards ,,verbindende Klammer“ stark beeinträchtigt $^{51}$.

Auch die Bedeutung des Rahmenkonzepts als Rechtfertigungsgrundlage des IASB im Rahmen der Standardsetzung wird dadurch relativiert, dass die im Rahmenkonzept kodifizierten Zwecke und Prinzipien ebenfalls von den Rechnungslegungsadressaten verwendet werden, um ihre - teils auch vom IASB abweichenden - Ansichten und Interessen im Standardsetzungsprozess zu stützen. ${ }^{52}$ Aufgrund unterschiedlicher Adressaten sowie des jeweiligen ökonomischem Umfelds können die Anforderungen an einzelne Rechnungslegungsvorschriften stark divergieren. ${ }^{53}$ Inwieweit die festgelegten Zwecke und Prinzipien inhaltlich präzisiert sind oder Ermessensspielräume erlauben, ist jedoch wesentlich für die normative Gültigkeit des Rahmenkonzepts.

\subsection{Rechnungslegungszwecke gemäß IFRS-Rahmenkonzept}

\subsection{1 Konkretisierung der Informationsfunktion durch die} Entscheidungsnützlichkeit als Fundamentalzweck

Das vom IASB verfolgte Ziel der Entwicklung von Rechnungslegungsstandards zur Förderung der Transparenz, Rechenschaft und Effizienz von Finanzmärkten wird durch den Informationszweck konkretisiert. ${ }^{54}$ Der Fokus auf die Informationsfunktion soll dabei unterschiedliche Informationsbedürfnisse erfüllen. Neben dem Zweck der Selbstinformation und Rechenschaft besteht das Erfordernis kapitalmarktorientierter Informationsvermittlung, ${ }^{55}$ das im Rahmenkonzept durch den Fundamentalzweck der Entscheidungsnützlichkeit normiert ist. ${ }^{56}$ Der Rechnungslegungszweck besteht demnach in der Vermittlung von Finanzinformationen über das berichtende Unternehmen, die nützlich für bestehende und potenzielle Investoren, Kreditgeber und andere Gläubiger sind, um Investitions- und Ressourcenallokationsentscheidungen treffen zu können. ${ }^{57}$ Im Zuge der Rahmenkonzeptüberarbeitung (2010) wurde der Adressatenkreis auf die Kapitalgeber als

\footnotetext{
${ }^{51}$ Vgl. Ballwieser (2005), S. 744 (auch Zitat).

52 Vgl. anhand des Rahmenkonzepts des FASB Storey (2003), S. 61 f.

${ }^{53}$ Vgl. Rost (1991), S. 80 f.; Homfeldt (2013), S. 81 f.

${ }^{54}$ Vgl. IFRS Constitution, Rz. 2 (a); CF (2018), SP1.5.

${ }^{55}$ Vgl. Pelger (2012), S. 59; ausführlich Sunder (1999), S. 23-29.

56 Vgl. Wich (2009), S. 13.

${ }^{57} \mathrm{Vgl}$. CF (2018), 1.2.
} 
primäre Nutzergruppe beschränkt. ${ }^{58}$ Als entscheidungsnützlich gelten Informationen, die eine Einschätzung über die Ertragserwartung der Investition sowie den Zeitpunkt, die Höhe und die Unsicherheit der künftigen Nettomittelzuflüsse des Unternehmens ermöglichen. ${ }^{59} \mathrm{Um}$ die finanzielle Situation eines Unternehmens beurteilen zu können, bedarf es daher Informationen über die Ressourcen und Ansprüche gegen das Unternehmen und Änderungen dieser sowie solcher, die Auskunft darüber geben, inwieweit das Management seine Verantwortung für die Nutzung und den Einsatz der ihm anvertrauten Unternehmensressourcen erfüllt hat. ${ }^{60}$

Während die Entscheidungsnützlichkeit in IAS 1.9 allgemein durch die Nützlichkeit für wirtschaftliche Entscheidungen definiert wird, ${ }^{61}$ konkretisiert das Rahmenkonzept die Funktion konkret im Sinne der Bewertungsnützlichkeit ${ }^{62}$. Informationen über die Vermögens-, Finanz- und Ertragslage sowie die Managementleistung sollen demnach als Grundlage für Investitionsentscheidungen dienen. ${ }^{63}$ Die nutzerorientierte Ausrichtung vermag die Mehrdeutigkeit ${ }^{64}$ darüber, welche Informationen als bewertungsnützlich gelten, indes nicht zu beschränken, da die Interessen von Eigen- und Fremdkapitalgebern als primäre Adressaten durchaus heterogen sind. ${ }^{65}$ Obwohl das IASB auf die unterschiedlichen Informationsbedürfnisse der Nutzergruppe im Rahmenkonzept hinweist, ${ }^{66}$ werden Entscheidungen im Standardsetzungsprozess häufig durch eine typisierte Entscheidungsnützlichkeit im Sinne der Nutzer ${ }^{67}$ begründet. Hierdurch wird eine Objektivität der Entscheidungsnützlichkeit suggeriert, die jedoch adressatenunabhängig nicht besteht. ${ }^{68}$ Vielmehr wird das Konzept der Entscheidungsnützlichkeit durch das IASB - und anderen Interessengruppen - nutzerorientiert plausibilisiert und dabei gleichsam subjektiv interpretiert. ${ }^{69}$ Die Entscheidungsnützlichkeit für

\footnotetext{
${ }^{58}$ Vgl. CF (2018), 1.5; CF (2018), BC1.9-BC1.13.

${ }^{59} \mathrm{Vgl}$. CF (2018), 1.3.

${ }^{60} \mathrm{Vgl}$. CF (2018), 1.4.

${ }^{61}$ Vgl. ebenso Framework (1989), 14; CF (2018), BC1.27.

${ }^{62}$ Vgl. Pelger (2012), S. 61; Gassen u. a. (2008), S. 876 f.

${ }^{63}$ Vgl. CF (2018), 1.13-1.23.

${ }^{64}$ Vgl. CF (2018), BC2.7; Lee (2015), S. 117; zu den qualitativen Anforderungen an entscheidungsnützliche Informationen Abschnitt 2.1.1.2.2.4 und 2.1.1.2.2.5.

65 Vgl. Homfeldt (2013), S. 87; Pelger (2012), S. 56-58; Young (2006), S. 589-595.

66 Vgl. CF (2018), 1.8.

${ }^{67}$ Vgl. Young (2006), S. 593; Pelger (2012), S. 58; Stenka/Jaworska (2019), S. 3 f.

68 Vgl. Ballwieser (2005), S. 733; Berndt (2005), S. 50 f.

${ }^{69}$ Vgl. Ballwieser (2005), S. 733; Botzem (2010), S. 176; Pelger (2012), S. 58.
} 
den Nutzer als Deduktionsgrundlage für die Standardsetzung stellt daher zunächst keine restriktive Basis für Wertungsentscheidungen dar, da sich durch Referenz auf den Nutzen für Kapitalgeber verschiedene Rechnungslegungsvorschriften rechtfertigen lassen. ${ }^{70}$

\subsection{2 Nachrangige Bedeutung der Rechenschaftsfunktion als Bestandteil entscheidungsnützlicher Informationen}

Die Informationsvermittlung zum Zwecke der Entscheidungsnützlichkeit umfasst neben bewertungsnützlichen Informationen auch Informationen über die Managementleistung. ${ }^{71}$ Das Management legt durch die bereitgestellten Informationen Rechenschaft über den Umgang mit den ihm anvertrauten Ressourcen ab, mithilfe derer die Kapitalgeber die Handlungen bewerten und Investitionsentscheidungen treffen können, sog. Stewardship-Funktion. ${ }^{72}$ Im Gegensatz zum alten Rahmenkonzept (1989) und IAS 1.9, der die Stewardship-Funktion weiterhin als eigenständigen Zweck von Abschlüssen neben dem Ziel der Vermittlung entscheidungsnützlicher Informationen formuliert, wurde der Verweis auf die Rechenschaft im Zuge der Rahmenkonzeptüberarbeitung (2010) zwischenzeitlich gänzlich eliminiert, aufgrund der Forderung verschiedener Interessengruppen indes in 2018 wieder aufgenommen. ${ }^{73}$ Trotz der stärkeren Betonung der Rechenschaft als Bestandteil der Ressourcenallokationsentscheidungen sowie der expliziten Verwendung des Stewardship-Begriffs stellt die Rechenschaftsfunktion im Rahmenkonzept keinen separaten Zweck neben der Entscheidungsnützlichkeit dar. $^{74}$

Die Bestrebung des IASB, die Zwecke der Entscheidungsnützlichkeit und Rechenschaft zu vereinen, beruht auf ihrer gleichgerichteten Funktion, Informationsasymmetrien zwischen dem berichtenden Unternehmen und den Adressaten $\mathrm{zu}$ verringern. ${ }^{75}$ So dient die Beurteilung der Rechenschaftserfüllung auch als Indikator für die Effektivität des Managements Renditen zu erzielen und somit als Entscheidungsgrundlage für künftige Investitionen. ${ }^{76}$ Der Rechenschaftszweck erfüllt jedoch auch eine der Kontrolle und Anreizzwecken von

\footnotetext{
${ }^{70}$ Vgl. Pelger (2009), S. 159-162.

${ }^{71} \mathrm{Vgl}$. CF (2018), 1.4.

${ }^{72}$ Vgl. CF (2018), 1.20-1.22.

${ }^{73}$ Vgl. CF (2018), BC1.32-BC1.41; zur Diskussion der Stewardship-Verankerung im Zuge der Rahmenkonzeptüberarbeitung Lennard (2007), S. 52-66; Pelger (2016), S. 55-68.

${ }^{74}$ Vgl. CF (2018), BC.1.34 f.; Kirsch (2018), S. 618; Pelger (2020), S. 37.

75 Vgl. Hettich (2006), S. 7-12; Coenenberg/Straub (2008), S. 22.

${ }^{76}$ Vgl. Zeff (2013), S. 313.
} 
Prinzipal-Agenten-Beziehungen dienende Funktion, die auf Basis vergangenheitsorientierter Informationen eine Verhaltenssteuerung ex ante ermöglicht. ${ }^{77}$ Diese „komplementären, aber nicht identischen Zwecke[.]“ verlangen eine Wertungsentscheidung über das Verhältnis beider Zwecke zueinander, ${ }^{78}$ da sich aus den jeweiligen Zwecken unterschiedliche Anforderungen an die zu vermittelnden Informationen ergeben. ${ }^{79}$ „Die nachrangige Nennung von Rechenschaft gegenüber Entscheidungsunterstützung lässt sich [daher] als ein die Grenzen der gleichzeitigen Erfüllung beider Zwecke deutlich machender Kompromiss verstehen “80, der sich auch in den qualitativen Anforderungen des Rahmenkonzepts widerspiegelt. ${ }^{81}$

\subsection{Grundlegende qualitative Anforderungen des IFRS-Rahmenkonzepts}

\subsection{1 Anforderung der Relevanz als zweckadäquate Konkretisierung der Entscheidungsnützlichkeit}

Der Zweck der Entscheidungsnützlichkeit wird durch die grundlegenden Anforderungen der Relevanz und der glaubwürdigen Darstellung sowie den weiterführenden Anforderungen der Vergleichbarkeit, Nachprüfbarkeit, Zeitnähe und Verständlichkeit konkretisiert. ${ }^{82}$ Informationen über wirtschaftliche Ereignisse, die für Nutzer entscheidungsnützlich sein können, müssen dabei zunächst die Anforderung der Relevanz und anschließend der glaubwürdigen Darstellung erfüllen, erst hiernach sind die weiterführenden Anforderungen zu prüfen. ${ }^{83}$ Das IASB legt hierdurch eine Hierarchie mit der Relevanz als primäre Anforderung fest. ${ }^{84}$ „Mit der Implementierung eines Evaluierungsschemas für die Findung von Bilanzierungslösungen bindet sich der Standardsetzer [jedoch] faktisch an

\footnotetext{
77 Vgl. grundlegend zu den Unterschieden zwischen Entscheidungsnützlichkeit und Rechenschaft Watts (1977), S. 63; Gjesdal (1981), S. 209-211; Miller/Oldroyd (2018), S. 71-75.

${ }^{78}$ Vgl. Pelger (2009), S. 160 (auch Zitat).

${ }^{79}$ Vgl. Gjesdal (1981), S. 209-211; Whittington (2008), S. 144 f.; Coenenberg/Straub (2008), S. 18-21.

${ }^{80}$ Ballwieser (2014), S. 470.

${ }^{81}$ Vgl. kritisch zur konzeptionellen Umsetzung Dehmel u. a. (2018), S. 1706 f.

${ }^{82} \mathrm{Vgl}$. CF (2018), 2.4.

${ }^{83}$ Vgl. CF (2018), 2.20 f.; CF (2018), BC2.9-2.11.

${ }^{84}$ Vgl. Whittington (2008), S. 146; Pelger (2012), S. 148 f.; Wawrzinek/Lübbig (2016), § 2, Rz. 57.
} 
dessen Anwendung. “85 Einer der Hauptzwecke des Standardsetzungsprozesses ist es daher die Ansichten der Nutzer über die Relevanz der auf Basis eines veröffentlichten Standardentwurfs vermittelten Informationen einzuholen. ${ }^{86}$ Gleichzeitig ermöglicht die Vorgabe anderen Interessengruppen den Standardsetzungsprozess gezielter durch Verweis auf die jeweilige Anforderung und ihren Stellenwert zu beeinflussen, ${ }^{87}$ insbesondere wenn die einzelnen Anforderungen einen Auslegungsspielraum eröffnen ${ }^{88}$.

Die Anforderung der Relevanz ist definiert durch die Fähigkeit Entscheidungen von Nutzern beeinflussen zu können. ${ }^{89}$ Relevante Informationen müssen demnach wertvoll für die Einschätzung zukünftiger Ergebnisse sein, mithin einen vorhersagenden Wert besitzen, sowie eine Beurteilung vergangener Einschätzungen ermöglichen und folglich auch einen bestätigenden Wert haben. ${ }^{90}$ So können bspw. Angaben über Umsatzerlöse des aktuellen Geschäftsjahres sowohl vergangenheitsorientierte Informationen über vorherige Jahre als auch gleichzeitig Prognosen für kommende Jahre liefern. ${ }^{91}$ Der vorhersagende Wert einer Informationen bezieht sich daher nicht auf die Vorhersagbarkeit im Sinne einer statistischen Genauigkeit einer Prognose, sondern vielmehr auf die Verwendbarkeit zur Einschätzung künftiger Ereignisse. ${ }^{92}$ Aufgrund des primären Adressatenkreises der Kapitalgeber scheint besonders dieser auf den prospektiven Informationsgehalt gerichtete Aspekt der Relevanz sinnvoll, um eine Einschätzung der künftigen Nettozuflüsse und somit der Rentabilität einer möglichen Investition zu ermöglichen. ${ }^{93}$ Die Festlegung der Relevanz als primäre Anforderung stellt folglich eine zweckadäquate Konkretisierung der Entscheidungsnützlichkeit dar, vermag den adressatenabhängigen Auslegungsspielraum jedoch nicht objektiv zu begrenzen.

\footnotetext{
${ }^{85}$ Hoffmann/Detzen (2012), S. 55.

${ }^{86} \mathrm{Vgl}$. CF (2018), BC2.14.

${ }^{87}$ Vgl. Hoffmann/Detzen (2012), S. 55.

${ }^{88}$ Vgl. Homfeldt (2013), S. 93.

${ }^{89} \mathrm{Vgl}$. CF (2018), 2.6.

${ }^{90} \mathrm{Vgl}$. CF (2018), 2.7-2.9.

${ }^{91} \mathrm{Vgl}$. CF (2018), 2.10.

92 Vgl. CF (2018), BC2.17.

${ }^{93}$ Vgl. Coenenberg/Straub (2008), S. 20.
} 


\subsection{2 Objektivierungstendenz der Anforderung der glaubwürdigen Darstellung}

\subsection{2.1 Wirtschaftliche Betrachtungsweise}

Um dem Objektivierungserfordernis entscheidungsnützlicher Informationen gerecht zu werden, besteht neben der Anforderung der Relevanz die zweite grundlegende Anforderung der glaubwürdigen Darstellung. ${ }^{94}$ Die glaubwürdige Darstellung ersetzt - trotz zahlreicher Kritik verschiedener Interessengruppen die im alten Rahmenkonzept (1989) und in einzelnen IFRS-Standards bestehende Anforderung der Verlässlichkeit, die als „effective filter“ die Informationsvermittlung objektivierungsbedingt zugunsten der Nachprüfbarkeit von Informationen einschränkte. ${ }^{95}$ Das Erfordernis der Nachprüfbarkeit ist nach Ansicht des IASB der glaubwürdigen Darstellung wirtschaftlicher Verhältnisse nachgelagert und nur ergänzend zu berücksichtigen. ${ }^{96}$ Informationen sollen demnach nicht nur die rechtliche Form, sondern die wirtschaftliche Substanz relevanter Ereignisse abbilden. ${ }^{97}$ Die rechtliche Anknüpfung der Vermögenswert- und Schuldendefinition an vertragliche sowie gesetzliche Rechte und Pflichten ${ }^{98}$ wirkt im Sinne einer rechtlichen Betrachtungsweise zunächst objektivierend ${ }^{99}$. Diese Objektivierung wird jedoch insofern durch die wirtschaftliche Betrachtungsweise zurückgedrängt, als vertragliche Vereinbarungen ohne wirtschaftlichen Gehalt nicht zu berücksichtigen sind und potenzielle Vermögenswerte sowie Schulden auch durch faktische Verpflichtungen konkretisiert werden können. ${ }^{100}$ Das IASB normiert im Rahmenkonzept keine von der zugrundeliegenden Rechtsstruktur losgelöste wirtschaftliche Betrachtungsweise ${ }^{101}$, sondern verlangt nur dann eine Abweichung

\footnotetext{
${ }^{94}$ Vgl. CF (2018), 2.5; CF (2018), BC2.22.

95 Vgl. CF (2018), 2.12; CF (2018), BC2.22-2.30 (auch Zitat, BC2.28); zur Bedeutung und zum Verhältnis von Verlässlichkeit, glaubwürdige Darstellung und Objektivierung Whittington (2008), S. 146-148; ebenso sowie zur Kritik der Interessengruppen im Zuge der Rahmenkonzeptüberarbeitung (2010) Erb/Pelger (2015a), S. 21-34.

96 Vgl. CF (2018), 2.12; CF (2018), 2.23; CF (2018), BC2.22-2.30.

${ }^{97} \mathrm{Vgl}$. CF (2018), 2.12.

${ }^{98}$ Vgl. CF (2018), 4.7; CF (2018), 4.31; CF (2018), 4.59.

${ }^{99}$ Vgl. zur Objektivierungsfunktion der rechtlichen Betrachtungsweise Moxter (2003), S. 324 f.; Beisse (1984), S. 2.

100 Vgl. CF (2018), 4.59-4.61; Schmidt (2018), S. 1.

${ }^{101} \mathrm{Vgl}$. zu unterschiedlichen Ausgestaltungsmöglichkeiten des Grundsatzes der wirtschaftlichen Betrachtungsweise Florstedt u. a. (2015), S. 376-384; Alexander u. a. (2018), S. 19631971.
} 
von der Darstellung der rechtlichen Form, wenn eine Vereinbarung keine wirtschaftliche Bedeutung hat, wie bspw. im Fall eines nicht bindenden Vertrags. ${ }^{102}$ Im Einzelfall kann die Anforderung der glaubwürdigen Darstellung daher jedoch eine objektivierte Informationsvermittlung im Sinne der Rechtssicherheit durch eine subjektiv relevantere Abbildung des wirtschaftlichen Gehalts überlagern.

\subsection{2.2 Vorsichtsprinzip}

Die Anforderung der glaubwürdigen Darstellung wird zusätzlich durch die Eigenschaften der Vollständigkeit, Neutralität und Freiheit von Fehlern konkretisiert. ${ }^{103}$ Vollständigkeit erfordert die Vermittlung sowie Beschreibung und Erläuterung aller für den Adressaten erforderlichen Informationen, um das dargestellte Ereignis zu verstehen. ${ }^{104}$ Für eine neutrale Darstellung müssen die Auswahl und Vermittlung von Informationen zudem ohne verzerrende Einflüsse und somit manipulationsfrei erfolgen. ${ }^{105}$ Während das im alten Rahmenkonzept (1989) mit dem Neutralitätsgrundsatz gleichgestellte Vorsichtsprinzip zunächst aufgrund der Unvereinbarkeit mit der im Zuge der Rahmenkonzeptüberarbeitung (2010) veränderten Stellung der Anforderung der Neutralität abgeschafft wurde, löste das IASB bei der Finalisierung des Rahmenkonzepts in 2018 den Konflikt durch Konkretisierung des Vorsichtsprinzips als Bestandteil des Neutralitätsgrundsatzes. ${ }^{106}$ Das Vorsichtsprinzip soll als ein der Neutralität untergeordnetes Kriterium diese stärken, indem es eine vorsichtige Ausübung von Entscheidungen unter Unsicherheit verlangt. ${ }^{107}$ Das IASB normiert mit dieser sog. ,,cautious prudence" anstelle eines konservativen, risikoantizipierenden Vorsichtsprinzips ein mit dem Neutralitätsgrundsatz in Einklang stehendes Vorsichtprinzip, das die Tendenz zu optimistischer oder $\mathrm{zu}$ negativer Managemententscheidungen und daraus resultierender Verzerrungen in Form von Über- oder Unterbewertungen verhindern soll. ${ }^{108}$ Eine einseitig vorsichtige Bewertung von Vermögenswerten, Schulden und Erträgen im Sinne eines asymmetrischen Vorsichtsprinzips ist nach Ansicht des IASB nicht immer mit der Anforderung der Relevanz und

$102 \mathrm{Vgl} . \mathrm{CF}$ (2018), $4.60 \mathrm{f}$.

103 Vgl. CF (2018), 2.13.

104 Vgl. CF (2018), 2.14.

105 Vgl. CF (2018), 2.15.

106 Vgl. Pelger (2020), S. 38 f.

$107 \mathrm{Vgl.} \mathrm{CF}$ (2018), 2.16.

${ }^{108}$ Vgl. CF (2018), 2.16 f.; CF (2018), BC2.39 (auch Zitat); zum Unterschied zwischen „,conservatism“ und ,prudence“ Barker (2015), S. 516-521. 
der glaubwürdigen Darstellung vereinbar. ${ }^{109}$ Gleichzeitig können asymmetrisch vorsichtige Regelungen auf Einzelstandardebene, wie bspw. die unterschiedlichen Wahrscheinlichkeitsschwellen zum Ansatz von Eventualforderungen und -verbindlichkeiten nach IAS 37 oder die Einrechnung variabler Vergütungen nach IFRS 15, jedoch im Einzelfall aufgrund der Vermittlung relevanterer Informationen durch das IASB gerechtfertigt werden. ${ }^{10}$ Den Widerspruch zwischen neutraler Darstellung und konservativer Bilanzierung durch eine risikoneutrale Ausrichtung des Vorsichtsprinzips zu lösen, kann für eine Deduktion konsistenter Einzelnormen daher nicht gänzlich überzeugen. ${ }^{11}$

\subsection{2.3 Bewertungsunsicherheiten}

Eine glaubwürdige Darstellung verlangt zuletzt die fehlerfreie Beschreibung eines Ergebnisses sowie die fehlerfreie Auswahl und Anwendung der für die Informationsvermittlung angewandten Verfahren. ${ }^{112}$ Bewertungsunsicherheiten, die bei einer angemessenen Schätzung nicht beobachtbarer Werte auftreten, sind bei der Beurteilung der Fehlerfreiheit zu berücksichtigen; sie stehen der Entscheidungsnützlichkeit nicht entgegen, sofern sie klar und deutlich beschrieben und erläutert werden. ${ }^{113}$ Eine Schätzung kann zwar auch bei hoher Unsicherheit relevante Informationen liefern, ihre glaubwürdige Darstellung wird jedoch vom Ausmaß der Bewertungsunsicherheiten beeinflusst. ${ }^{114}$ Das IASB verweist im Zuge dessen auf die Nachprüfbarkeit als ergänzende Anforderung und stärkt damit die Bedeutung der Verlässlichkeit von Informationen. ${ }^{115}$ Die Berücksichtigung von Bewertungsunsicherheiten als Teilaspekt der glaubwürdigen Darstellung führt somit zu einer objektivierungsbedingten Restriktion, die im Einzelfall eine Abwägung zwischen der Anforderung der Relevanz und glaubwürdigen Darstellung erfordert ${ }^{116}$. Die adäquate Gewichtung beider Anforderungen ist zwar grundsätzlich konsequent im Hinblick auf die in der Zwecksetzung der Entscheidungsnützlichkeit integrierte Rechenschaftsfunktion sowie des dafür notwendigen

\footnotetext{
${ }^{109}$ Vgl. CF (2018), BC2.42.

${ }^{110}$ Vgl. CF (2018), 2.17; CF (2018), BC2.44 f.; zur Analyse vorsichtiger Regelungen auf Einzelstandardebene Barker/McGeachin (2015), S. 183-197.

${ }^{111}$ Vgl. Pelger (2020), S. 39; Dehmel u. a. (2018), S. 1707 f.; Barker/McGeachin (2015), S. 181 f.; Erb/Pelger (2015b), S. 1063.

112 Vgl. CF (2018), 2.18.

113 Vgl. CF (2018), 2.19.

$114 \mathrm{Vgl}$. CF (2018), BC2.48 f.

115 Vgl. CF (2018), BC2.48 (a); Erb/Pelger (2018b), S. 877.

116 Vgl. CF (2018), 2.22; CF (2018), BC2.52-BC2.56.
} 
Objektivierungserfordernisses ${ }^{117}$. Das IASB setzt mit der Einführung der Bewertungsunsicherheiten im Rahmenkonzept in 2018 insofern jedoch wieder einen im alten Rahmenkonzept (1989) und im Zuge der Rahmenkonzeptüberarbeitung (2010) zunächst abgeschafften Trade-Off um, anstatt eine klare Abgrenzung zwischen der Verlässlichkeit und glaubwürdigen Darstellung zu schaffen. ${ }^{118}$

\subsection{Weiterführende qualitative Anforderungen des IFRS-Rahmenkonzepts}

Die weiterführenden Anforderungen der Vergleichbarkeit, Nachprüfbarkeit, Zeitnähe und Verständlichkeit können den Zweck der Entscheidungsnützlichkeit allein nicht erfüllen; sie erhöhen jedoch die Nützlichkeit relevanter und glaubwürdig dargestellter Informationen und dienen als Entscheidungshilfe, sofern zwei Möglichkeiten der Informationsvermittlung die Anforderung der Relevanz und glaubwürdigen Darstellung gleichermaßen erfüllen. ${ }^{119}$ Jede der weiterführenden Anforderungen sollte möglichst umfassend erfüllt werden, wobei keine Anwendungshierarchie vorgegeben wird und einzelne Anforderungen im Einzelfall auch gegeneinander abgewogen werden müssen. ${ }^{120}$

Informationen sind demnach entscheidungsnützlicher, wenn sie den Adressaten eine unternehmens- und periodenübergreifende Vergleichbarkeit ermöglichen, um Ähnlichkeiten und Unterschiede zwischen Sachverhalten identifizieren und verstehen zu können. ${ }^{121}$ Hiervon abzugrenzen ist der Grundsatz der Stetigkeit, der die Verwendung gleicher Methoden für gleiche Sachverhalte verlangt und damit zur Vergleichbarkeit der Informationen beiträgt, sowie das Erfordernis der Einheitlichkeit. ${ }^{122}$ Ein gewisses $\mathrm{Ma}$ an Vergleichbarkeit wird normalerweise bereits durch die Erfüllung der beiden grundlegenden Anforderungen erreicht und durch den Verzicht auf Wahlrechte erhöht. ${ }^{123}$ Zudem soll die Informationsvermittlung durch eine deutliche und prägnante Klassifizierung, Beschreibung und Darstellung verständlich sowie zeitnah erfolgen. ${ }^{124}$ Weder die Anforderung der Verständlichkeit noch der Zeitnähe stellen eine Restriktion der Relevanz dar,

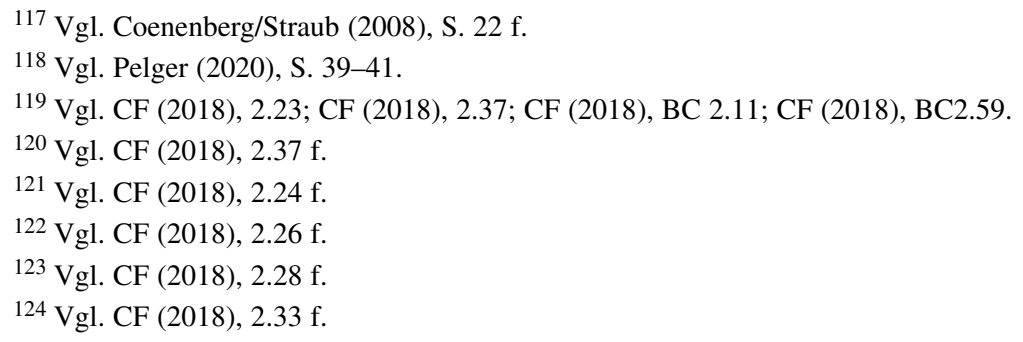

117 Vgl. Coenenberg/Straub (2008), S. 22 f.

118 Vgl. Pelger (2020), S. 39-41.

${ }^{119}$ Vgl. CF (2018), 2.23; CF (2018), 2.37; CF (2018), BC 2.11; CF (2018), BC2.59.

${ }^{120} \mathrm{Vgl} . \mathrm{CF}$ (2018), $2.37 \mathrm{f}$.

$121 \mathrm{Vgl}$. CF (2018), $2.24 \mathrm{f}$.

$122 \mathrm{Vgl} . \mathrm{CF}$ (2018), $2.26 \mathrm{f}$.

$123 \mathrm{Vgl} . \mathrm{CF}$ (2018), $2.28 \mathrm{f}$.

${ }^{124} \mathrm{Vgl}$. CF (2018), $2.33 \mathrm{f}$. 
weshalb Informationen über komplexe Sachverhalte, die nicht leicht verständlich sind oder nur verzögert dargestellt werden können, nicht ausgelassen werden dürfen. ${ }^{125}$

Die Vertrauenswürdigkeit glaubwürdig dargestellter Informationen wird durch die Anforderung der Nachprüfbarkeit unterstützt, die die Verifizierbarkeit durch Dritte verlangt. ${ }^{126}$ Als nachprüfbar gelten demnach Informationen, die direkt beobachtbar sind oder indirekt durch vergleichbare Methoden ermittelt werden können. ${ }^{127}$ Vor allem zukunftsgerichtete Informationen erfordern aufgrund ihrer häufig schwierigen Nachprüfbarkeit zusätzliche Angaben. ${ }^{128}$ Die Anforderung der Nachprüfbarkeit entspricht damit der im alten Rahmenkonzept bestehenden Anforderung der Verlässlichkeit. ${ }^{129}$ Obwohl die Berücksichtigung von Bewertungsunsicherheiten unter der Anforderung der glaubwürdigen Darstellung zu einem gewissen Grad an Objektivierung führt, kann das Erfordernis der Verlässlichkeit aufgrund der Einordnung der Nachprüfbarkeit als weiterführende Anforderung der Bandbreite subjektiver Werte, die entscheidungsnützlich sind, nur begrenzt entgegenwirken. ${ }^{130}$

Im Rahmenkonzept werden somit zwar wesentliche qualitative Anforderungen festgelegt, durch deren Konkretisierung und Abwägung die Entwicklung konsistenter Einzelstandards erfolgen soll. ${ }^{131}$ Die einzelnen Anforderungen sind jedoch primär der Anforderung der Relevanz untergeordnet und konfligieren teilweise miteinander, wodurch sie zunächst keine objektiv restriktive normative Grundlage für eine deduktive Standardsetzung vorgeben ${ }^{132}$ und dadurch Argumentationsspielraum in der Standardsetzung ermöglichen. Dieser zweckgerechte Kompromiss verdeutlicht, dass die Standardsetzung nicht nur technischer Natur ist $^{133}$. Rechnungslegungsnormen haben verschiedene ökonomische und soziale Konsequenzen für unterschiedliche Interessengruppen, ${ }^{134}$ weshalb vom IASB

\footnotetext{
125 Vgl. CF (2018), 2.35; CF (2018), BC2.65; CF (2018), BC2.68.

126 Vgl. CF (2018), 2.30; CF (2018), BC2.60.

127 Vgl. CF (2018), 2.31.

128 Vgl. CF (2018), 2.32.

${ }^{129}$ Vgl. CF (2018), BC2.60 f.

${ }^{130}$ Vgl. zur Forderung der Verankerung der Nachprüfbarkeit als separate Anforderung Pelger (2020), S. $40 \mathrm{f}$.

131 Vgl. Bennett u. a. (2006), S. 198.

132 Vgl. Wich (2009), S. 48; Macve (1997), S. 77.

${ }^{133}$ Vgl. Rappaport (1977), S. 89 f.; Watts/Zimmerman (1978), S. 112 f.

${ }^{134}$ Vgl. Prakash/Rappaport (1977), S. 35-38; Zeff (1978), S. 61.
} 
im Rahmen der Standardsetzung auch praktische Erwägungen berücksichtigt werden. ${ }^{135}$

\subsubsection{Induktive Normermittlung}

\subsubsection{Pragmatischer Charakter von Rechnungslegungsvorschriften}

Für den Zweck der Entscheidungsnützlichkeit ist der Wahrheitsgehalt der bereitgestellten Informationen wesentlich. ${ }^{136}$ Fraglich ist daher, welche Wahrheitskonzeption den IFRS zugrunde liegt. ${ }^{137}$ Gemäß dem Grundsatz der sog. fair presentation ${ }^{138}$ in IAS 1.15 und der im alten Rahmenkonzept bestehenden Anforderung des sog. True-and-Fair-View ${ }^{139}$ sollen die vermittelten Informationen die Vermögens-, Finanz- und Ertragslage eines Unternehmens den tatsächlichen Verhältnissen entsprechend darstellen. Die Abbildung der tatsächlichen Verhältnisse erfordert dabei eine mit den Definitions- und Ansatzkriterien des Rahmenkonzepts übereinstimmende glaubwürdige Darstellung der Auswirkungen von Geschäftsvorfällen, was annahmegemäß durch Anwendung der IFRS und gegebenenfalls zusätzlicher Angaben erreicht wird (IAS 1.15). Die einzelnen Rechnungslegungsvorschriften fungieren demnach als Abbildungsregeln der tatsächlichen wirtschaftlichen Sachverhalte, ${ }^{140}$ demzufolge die Realitätsbezogenheit zum Maßstab für eine wahre Rechnungslegung wird ${ }^{141}$. Entsprechend diesem korrespondenztheoretischen Wahrheitsverständnisses ${ }^{142}$ gilt eine Information als

\footnotetext{
135 Vgl. Preface (2019), Rz. 10.

${ }^{136}$ Vgl. Kam (1990), S. 53.

${ }^{137}$ Vgl. zur Diskussion unterschiedlicher philosophischer Prämissen der Rechnungslegung Shapiro (1997), S. 167-179.

${ }^{138}$ Vgl. zur Konkretisierung des Konzepts der „fair presentation“ Najderek (2009), S. 175198.

${ }^{139}$ Vgl. Framework (1989), 46. Gemäß CF (2018), BC3.44 ist die Vermittlung eines den tatsächlichen Verhältnissen entsprechenden Bildes eine Umschreibung für Informationen, die die qualitativen Anforderungen erfüllen.

140 Vgl. Lukka (1990), S. 239.

${ }^{141}$ Vgl. Schmitz (2012), S. 199.

142 Vgl. zur Korrespondenztheorie der Wahrheit Sellars (1992), S. 300-336; Gloy (2004), S. 92-167.
} 
objektiv wahr, wenn sie mit der unabhängig von Individuen bestehenden Wirklichkeit übereinstimmt, sie folglich nachprüfbar ist und die Darstellung frei von subjektiven Einflüssen erfolgt. ${ }^{143}$

Inwiefern jedoch eine wirtschaftliche Realität objektiv besteht, ist fraglich, ${ }^{144}$ und besonders mit der im Rahmenkonzept verlangten entscheidungsnützlichen Informationsvermittlung schwer vereinbar, ${ }^{145}$ wonach Informationen im Hinblick auf ihren Realitätsbezug nicht tatsächlich wahr sein, sondern vom Adressaten als entscheidungsbeeinflussend angesehen werden müssen ${ }^{146}$. So weisen die qualitativen Anforderungen im Rahmenkonzept zwar Objektivierungstendenzen auf, ${ }^{147}$ sie zielen letztlich jedoch auf die Nützlichkeit einer Information ab, die sich subjektabhängig ${ }^{148}$ bzw. intersubjektiv ${ }^{149}$ anhand der primären Adressatengruppe bestimmt. Im Rahmen der Standardsetzung fließen dabei die Wertungen des IASB und bei der Standardanwendung die Wertungen der bilanzierenden Unternehmen ein. Entsprechend diesem pragmatischen Wahrheitsverständnis ${ }^{150}$ gilt eine Information als wahr, wenn ,ihre praktischen Konsequenzen eine [...] als wahr angesehene Erkenntnis für einzelne Individuen hat.“151 Maßgeblich ist somit die Funktionalität einer Norm. ${ }^{152}$ Die Forderung nach einer den tatsächlichen Verhältnissen entsprechenden Darstellung ist demnach pragmatisch zu konkretisieren; ${ }^{153}$ die praktische Bewährung von Rechnungslegungsvorschriften wird dabei zum Maßstab einer wahren Rechnungslegung. Dieser dem Fundamentalzweck der

\footnotetext{
${ }^{143}$ Vgl. Berndt (2005), S. 141-150.

144 Vgl. insgesamt Hines (1988); Lee (2006a), S. 46 f.; Schmitz (2012), S. 199-202.

145 Vgl. Lukka (1990), S. 249-254.

146 Vgl. Berndt (2005), S. $124 \mathrm{f}$.

${ }^{147} \mathrm{Vgl}$. Abschnitt 2.1.1.2.2.4 und 2.1.1.2.2.5.

148 Vgl. Berndt (2005), S. $151 \mathrm{f}$.

${ }^{149}$ Vgl. Archer (1997), S. 236; Alexander/Archer (2003), S. 6; zur Unterscheidung des ontologischen Wahrheitsbegriffs in physikalische Realität (rohe Tatsachen) und soziale Realität (institutionelle Tatsachen) Searle (1997), S. 11-39; zur Differenzierung der einzelnen „Schichten“ der Realität Mattessich (2014), S. 147-182.

${ }^{150}$ Vgl. zum Wahrheitsbegriff des Pragmatismus James (1992), S. 35-58; Gloy (2004), S. 222-226.

${ }^{151}$ Vgl. Berndt (2005), S. 163 (auch Zitat); Hines (1988), S. 257; Kam (1990), S. 488 i. V. m. S. 494.

152 Vgl. Schmitz (2012), S. 210 f.

${ }^{153}$ Vgl. Mattessich (1995), S. 7.
} 
Entscheidungsnützlichkeit immanente Anknüpfungspunkt zum Pragmatismus ${ }^{154}$ findet daher auch in der Normermittlung des IASB Berücksichtigung. ${ }^{155}$

\subsubsection{Induktion praktischer Bilanzierungsnormen}

\subsection{Ermittlung von Rechnungslegungsvorschriften auf Basis tatsächlicher Kaufmannsübung}

Im Gegensatz zum deduktiven Ansatz der Normermittlung, wonach Einzelnormen systemkohärent aus übergeordneten - normativ vorgegebenen - Zwecken abgeleitet werden, resultieren Einzelnomen unter dem induktiven Ansatz aus der Verallgemeinerung einzelner Tatsachen. ${ }^{156}$ Dies bedeutet, dass aus beobachtbaren Tatsachen Schlussfolgerungen gezogen und von diesen abstrahiert werden, wobei es sich im Fall empirischer Beobachtungen als Induktionsgrundlage um einen deskriptiven Ansatz handelt. ${ }^{157}$ Anknüpfungspunkt für die Ermittlung von Einzelnormen aus der „Natur der Sache“ bietet im Rahmen der Rechnungslegung die kaufmännische Übung, die tatsächlich feststellbar ist und zum Gewohnheitsrecht erstarken kann. ${ }^{158}$ Die Identifizierung von Rechnungslegungsvorschriften erfolgt demzufolge ausgehend von einzelnen Rechnungslegungspraktiken, die als „generally accepted“" gelten und auf Erfahrungen, Gewohnheiten und praktischer Notwendigkeit beruhen. ${ }^{159}$ Als solche stellen sie ,ein Konglomerat einzelner Grundsätze oder Regeln [dar]; sie sind also ohne den [für den deduktiven Ansatz charakteristischen] Systemcharakter." ${ }^{160}$ Ein derartiges auf Einzelfälle ausgerichtetes Regelwerk verlangt aufgrund fehlender abstrakter Prinzipien meist umfangreiche Einzelnormen. ${ }^{161}$ Auch in der IFRS-Standardsetzung findet sich der in Einklang mit dem Pragmatismus stehende induktive Einfluss ${ }^{162}$ - nicht zuletzt aufgrund des sich aus der privatrechtlichen Organisation des IASB ergebenden öffentlichen Standardsetzungsprozesses ${ }^{163}$ - im Rahmenkonzept und auf Einzelstandardebene wieder. ${ }^{164}$

154 Vgl. Beams (1969), S. 384.

155 Vgl. Hoogervorst (2012a), S. 4.

156 Vgl. Ijiri (1975), S. 5 f.

157 Vgl. Ijiri (1975), S. 6 f.

158 Vgl. Radbruch (1948), S. 160-162; Euler (1989), S. 12-16.

${ }^{159}$ Vgl. ASC 105, 1 (auch Zitat); Luttermann (1999), S. 66-69.

160 Beisse (1990), S. 502.

161 Vgl. Euler (2002), S. 876.

162 Vgl. Berndt (2005), S. 168-172.

163 Vgl. Abschnitt 2.2.2.

164 Vgl. zum induktiven Einfluss auf Rahmenkonzeptebene Walton (2018), S. 195. 


\subsection{Praktikabilitätsorientierte Einflüsse auf Rahmenkonzeptebene}

\subsection{Berücksichtigung der Unternehmensaktivität}

Das Rahmenkonzept beinhaltet neben übergeordneten Prinzipien auch detaillierte Beschreibungen, um als ,practical tool“ für die Standardsetzung nützlich zu sein. ${ }^{165}$ So ist die Art der Unternehmensaktivität zwar nicht als allgemeine Anforderung im Rahmenkonzept normiert, jedoch als Faktor bei der Wahl der Rechnungseinheit und Bewertungsmethode sowie der Klassifizierung der Abschlussposten einzubeziehen. ${ }^{166}$ Das IASB betont zudem, dass das Konzept der Unternehmensaktivitäten bei der Entwicklung neuer und der Überarbeitung bestehender Standards zu berücksichtigen ist und als solches auf Einzelstandardebene, bspw. in Form des Geschäftsmodells zur Klassifizierung von Finanzinstrumenten in IFRS 9 Finanzinstrumente, konkretisiert werden kann. ${ }^{167}$ Die Berücksichtigung von Unternehmensaktivitäten wird mit der höheren Relevanz von Informationen gerechtfertigt ${ }^{168}$ und die wirtschaftliche Betrachtungsweise damit gestärkt. Gleichzeitig erlaubt die Berücksichtigung von Unternehmensaktivitäten jedoch unternehmensspezifische Erwägungen im Standardsetzungsprozess, was als induktiver Einfluss gewertet werden kann.

\subsection{Wesentlichkeitsabwägung}

Die Anforderung der Relevanz umfasst auch den Aspekt der Wesentlichkeit, wonach Informationen nicht weggelassen, verschleiert oder fehlerhaft dargestellt werden dürfen, wenn dies die Entscheidung der Adressaten beeinflussen könnte. ${ }^{169}$ Die Wesentlichkeit von Informationen ist nicht durch einen quantitativen Schwellenwert determiniert, sondern basiert auf der Art und/oder Größe der Abschlussposten, auf die sich die Informationen beziehen, und ist daher unternehmensspezifisch zu bestimmen. ${ }^{170}$ Die Beurteilung soll sich dabei an den Bedürfnissen der primären Adressatengruppe orientieren (IAS 1.7). ${ }^{171}$ Die nutzerorientierte Anknüpfung ist konsequent im Hinblick auf den in der Zwecksetzung

165 Vgl. CF (2018), BC0.13 (auch Zitat).

${ }^{166}$ Vgl. CF (2018), BC0.31; zu den unterschiedlichen Auffassungen zum Verweis auf die Unternehmensaktivitäten auf Rahmenkonzeptebene Technical Staff (2016a), Agenda Paper 10 L, Rz. 5-12.

${ }^{167}$ Vgl. CF (2018), BC0.31 f.; zur Stärkung des Business-Modell-Ansatzes Barker/Teixeira (2018), S. 156-159.

168 Vgl. CF (2018), BC0.29.

${ }^{169} \mathrm{Vgl}$. CF (2018), 2.11; zur Verschleierung von Informationen gemäß IAS $1.30 \mathrm{~A}$ Bach/Schreiber (2019), S. 271.

${ }^{170}$ Vgl. CF (2018), 2.11.

171 Vgl. CF (2018), BC2.20; IAS 8.BC35. 
festgelegten Adressatenkreis; sie zielt gleichzeitig auf eine „Reduzierung des Umfangs der als wesentlich zu qualifizierenden Informationen." 172 Eine Konkretisierung der quantitativen und qualitativen Aspekte erfolgt indes nicht, weshalb die Wesentlichkeitsbeurteilung Ermessensspielräume eröffnet. ${ }^{173}$ Unwesentliche Informationen haben zwar keinen Einfluss auf die Entscheidungen der Adressaten; ${ }^{174}$ ein ,,information overload“ verringert jedoch den Informationsgehalt und erschwert die Verständlichkeit der Informationen (IAS 1.30A). ${ }^{175}$ Einzelne Angabepflichten und Rechnungslegungsmethoden sind daher nicht anzuwenden, wenn ihre Auswirkung unwesentlich ist (IAS 1.31; IAS 8.8). Die Einschätzung der Wesentlichkeit kann damit einzelne Rechnungslegungsvorschriften überlagern, wenn dies im Sinne entscheidungsrelevanter Informationen zu ,praxisgerechte[n] Lösungen“ führt, ${ }^{176}$ und zielt somit auf den praktischen Nutzen einer Information $a b^{177}$.

Da sich Wesentlichkeitsentscheidungen sowohl auf den Ansatz und die Bewertung als auch auf den Ausweis und die Darstellung von Abschlussposten auswirken, veröffentlichte das IASB 2017 ein sog. Practice Statement, das das Konzept der Wesentlichkeit konkretisiert und Unternehmen als Leitlinie dienen soll. ${ }^{178}$ Das Practice Statement beinhalt Anwendungshinweise und gibt ein mögliches Prüfverfahren vor, das auch quantitative und qualitative Faktoren beispielhaft präzisiert. ${ }^{179}$ Obwohl das Practice Statement nicht verbindlich anzuwenden ist, ${ }^{180}$ kann sich aufgrund der Bedeutung der Wesentlichkeit im Rahmen der IFRS-Anwendung sowie der Abschlussprüfung bei gleichzeitig fehlender Konkretisierung innerhalb der einzelnen Standards eine faktische Bindungswirkung ergeben ${ }^{181}$. Das Practice Statement dient als vom Rahmenkonzept und den Einzelstandards losgelöste Verlautbarung folglich der Praktikabilität des Wesentlichkeitskonzepts und ist somit nur schwer mit dem angestrebten deduktiven Ansatz vereinbar.

\footnotetext{
172 Vgl. Busch/Zwirner (2018), S. 14 (auch Zitat).

173 Vgl. Bach/Schreiber (2019), S. 270 f.

${ }^{174}$ Vgl. CF (2018), BC2.19.

175 Vgl. Morunga/Bradbury (2012), S. 48 f., 58 f. (auch Zitat, S. 48); grundlegend Schick u. a. (1990), S. 200-206; Eppler/Mengis (2004), S. 326-333.

176 Vgl. Lüdenbach u. a. (2016), § 1, Rz. 60 (auch Zitat).

${ }^{177}$ Vgl. Hopf (1983), S. 15.

178 Vgl. IFRS Practice Statement 2, IN2-IN5.

${ }^{179}$ Vgl. IFRS Practice Statement 2, Rz. 8-65.

${ }^{180}$ Vgl. IFRS Practice Statement 2, IN6; Hebestreit/Teitler-Feinberg (2018), S. 148.

${ }^{181}$ Vgl. Link (2018), S. 172.
} 
Die Erläuterung des IASB, dass ein Standardsetzer die Wesentlichkeit im Rahmen der Standardsetzung nicht berücksichtigt, da es sich um eine unternehmensspezifische Erwägung handelt, ${ }^{182}$ steht ebenfalls im Widerspruch zum konzeptionellen Anspruch des Rahmenkonzepts und zur tatsächlichen Standardsetzung des IASB. So wird in zahlreichen Einzelstandards - wenn auch teilweise implizit - auf den Wesentlichkeitsgrundsatz Bezug genommen. Neben den oben genannten Regelungen zur Nichtanwendung einzelner Vorschriften in IAS 1 Darstellung des Abschlusses und IAS 8 Rechnungslegungsmethoden, Änderungen von rechnungslegungsbezogenen Schätzungen und Fehler verlangt bspw. IAS 37.46 nur dann eine Abzinsung für Rückstellungen, wenn der Zinseffekt als wesentlich eingestuft wird. Bei der Identifizierung von Leistungsverpflichtungen gemäß IFRS 15 sollen Unternehmen beurteilen, ob einzelne Leistungsverpflichtungen in Einklang mit IAS 8 als unwesentlich einzustufen und folglich nicht separat zu bilanzieren sind. ${ }^{183}$ „Die [Wesentlichkeit] in dem Bereich der IAS/IFRS dient [...] [somit] der Begrenzung der Informationsmenge auf entscheidungsrelevante Informationen." 184 Obwohl das IASB Wesentlichkeitserwägungen auf die Ebene der Standardanwendung zu beschränken versucht, verbleibt unklar, inwiefern sich bereits im Rahmen der Standardsetzung durch Verweis auf die Wesentlichkeit als Teilaspekt der Relevanz Vereinfachungsüberlegungen begründen lassen.

\subsection{Kosten-Nutzen-Abwägung}

Die Beurteilung der Wesentlichkeit führt zu einer kosteneffizienten Anwendung von IFRS-Standards, da unwesentliche Informationen nicht dargestellt werden müssen und sich die Kosten der Aufstellung folglich reduzieren. ${ }^{185}$ Trotz dieses Zusammenhangs zwischen der Wesentlichkeit und der Wirtschaftlichkeit der Informationsvermittlung sind die sich durch die Anwendung einzelner Rechnungslegungsvorschriften ergebenden Kosten kein von Unternehmen in die Wesentlichkeitsbeurteilung einzubeziehender Faktor. ${ }^{186}$ Der Verzicht auf die Anwendung einer Regelung durch Verweis auf die Unverhältnismäßigkeit von Kosten und Nutzen ist im Rahmen der Standardanwendung nur dann zulässig,

\footnotetext{
182 Vgl. CF (2018), BC2.19.

${ }^{183}$ Vgl. IFRS 15.BC90; zu den Wesentlichkeitsabwägungen in IFRS 16 Fehrenbach/Schulte (2018), S. $1516 \mathrm{f}$.

${ }^{184}$ Mekat (2009), S. 102.

185 Vgl. Maier (2008), S. 41.

186 Vgl. IAS 1.BC36; IFRS Practice Statement 2, Rz. 37.
} 
wenn eine Rechnungslegungsvorschrift einen derartigen „Praktikabilitätsvorbehalt" explizit beinhaltet. ${ }^{187}$ So kann bspw. gemäß IAS 8.23 auf eine volle retrospektive Anpassung bei Änderung einer Rechnungslegungsmethode verzichtet werden, wenn die Ermittlung der Auswirkungen undurchführbar ist. Die Undurchführbarkeit ist anhand der für die Anwendung angemessenen Anstrengungen des Unternehmens zu bemessen (IAS 8.5) und impliziert damit eine Kostenerwägung. ${ }^{188}$

Während die Kosten einer Regelungsvorschrift auf Ebene der Standardanwendung von Unternehmen nur im Ausnahmefall zu berücksichtigen sind, hat das IASB hingegen bei jeder Standardsetzungsentscheidung eine Kosten-NutzenAbwägung durchzuführen. ${ }^{189}$ Rechnungslegungsvorschriften werden demzufolge dahingehend beurteilt, ob der Nutzen der vermittelten Informationen die Kosten der Informationsbereitstellung und -verwendung wahrscheinlich rechtfertigt, ${ }^{190}$ wodurch das IASB Praktikabilitätserwägungen explizit im Standardsetzungsprozess berücksichtigt. Der Kostenaspekt umfasst dabei sowohl die Kosten der Erstellung und Vermittlung von Informationen auf Seiten des Unternehmens als auch die vom Adressaten zu tragenden Kosten der sich daraus ergebenden niedrigeren Erträge sowie der Informationsverarbeitung bzw. der zusätzlichen Kosten der Informationsbeschaffung im Fall nicht vermittelter Informationen. ${ }^{191}$ Auch die ökonomischen und sozialen Konsequenzen einzelner Rechnungslegungsvorschriften sind hierbei zu berücksichtigen. ${ }^{192}$ Der Nutzen liegt indes in der erhöhten Vertrauenswürdigkeit der auf Basis von IFRS erstellen Informationen, die zu einer höheren Kapitalmarkteffizienz und geringeren Kapitalkosten sowie der Entscheidungsnützlichkeit für den einzelnen Adressaten beitragen. ${ }^{193}$

Bei der Standardentwicklung versucht das IASB die Ansichten unterschiedlicher Interessengruppen zur Beurteilung der erwarteten Art und Quantität von Nutzen und Kosten des jeweiligen Standards einzuholen. ${ }^{194}$ Das IASB strebt dabei keine unternehmensspezifische Kosten-Nutzen-Abwägung an; aufgrund von Unterschieden in der Unternehmensgröße oder speziellen Adressatenbedürfnissen

\footnotetext{
187 Vgl. Lüdenbach u. a. (2016), § 1, Rz. 60 (auch Zitat).

188 Vgl. zur geplanten Konkretisierung der Praktikabilitätsschwelle durch eine KostenNutzenSchwelle Ljubicic (2019), S. 138 f.

189 Vgl. CF (2018), 2.39.

190 Vgl. CF (2018), 2.39, 2.42.

191 Vgl. CF (2018), 2.40.

192 Vgl. Zeff (1978), S. 63; Collett (1995), S. 19, 22-24.

193 Vgl. CF (2018), 2.41.

${ }^{194}$ Vgl. CF (2018), 2.42.
} 
rechtfertigt die Beurteilung von Kosten und Nutzen jedoch nicht immer dieselben Regelungen für alle Unternehmen. ${ }^{195}$ Die für die Kosten-Nutzen-Abwägung erforderlichen Informationen erhält das IASB im Rahmen des jeweiligen Standardsetzungsprozesses durch Veröffentlichung der Standardentwürfe sowie durch Feldstudien und Konsultation mit relevanten Akteuren. ${ }^{196}$ Die möglichen Auswirkungen eines neuen oder überarbeiteten Standards, insbesondere auch im Hinblick auf die Implementierungs- sowie Folgekosten, werden als Effektanalyse separat zu dem jeweiligen Einzelstandard bzw. bei Standardentwürfen in den zugehörigen Basis for Conclusions veröffentlicht. ${ }^{197}$ Die Bezeichnung als Effektanalyse verdeutlicht dabei, dass es sich - nicht zuletzt aufgrund praktischer Schwierigkeiten besonders in Bezug auf die Nutzenermittlung - weniger um eine quantitative Gesamtbeurteilung als vielmehr um eine qualitative Einschätzung handelt. ${ }^{198}$

Im Rahmenkonzept ist die Kosten-Nutzen-Abwägung nicht als qualitative Anforderungen, sondern als eine dem Prozess der Informationsermittlung dienende Anforderung normiert. ${ }^{199}$ Die Beurteilung des Nutzens einer möglichen Rechnungslegungsvorschrift als zweistufiger Prozess, mit den qualitativen Anforderungen auf der ersten und der Kostenrestriktion auf der zweiten Stufe, kann aufgrund des ,vorherrschende[n] Sachzwang[s]“ der Kostenrestriktion zum Konflikt zwischen konzeptionell entscheidungsnützlichen und praktisch kostensinnvollen Rechnungslegungsvorschriften führen. ${ }^{200}$ Die Kosten-Nutzen-Abwägung ermöglicht daher - besonders aufgrund des Einbezugs von Interessengruppen - eine gezielte Einflussnahme zugunsten praxisgerechter Rechnungslegungsvorschriften. Im Rahmen der Standardsetzung können folglich Argumente der Einfachheit, Operationalität, Durchführbarkeit, Anwendbarkeit und Akzeptabilität angebracht und mit der Anforderung der Kostenrestriktion begründet werden. ${ }^{201}$ Trotz des konzeptionellen Anspruchs beinhaltet das Rahmenkonzept somit ,a shadow of current practice“ ${ }^{\text {202 }}$, der einem induktiven Einfluss entspricht.

\footnotetext{
195 Vgl. CF (2018), 2.43.

196 Vgl. Due Process Handbook, Rz. 3.76; CF (2018), BC3.48.

197 Vgl. Due Process Handbook, Rz. 3.77-3.81.

198 Vgl. Schipper (2010), S. 316 f.

${ }^{199}$ Vgl. CF (2018), BC2.73.

${ }^{200}$ Vgl. Maier (2008), S. 53-55 (auch Zitat); zur schwierigen Abgrenzbarkeit von technisch/konzeptionellen Anforderungen und ökonomischen Folgen Collett (1995), S. 20-22.

${ }^{201}$ Vgl. zur Berücksichtigung der Argumente im Rahmen der Kosten-Nutzen-Analyse CF (2018), BC2.71 f.

202 Walton (2018), S. 198.
} 


\subsection{Praktikabilitätsorientierte Einflüsse auf Einzelstandardebene}

Entsprechend dem vom IASB angestrebten deduktiven Ansatz soll die Entwicklung der Einzelstandards grundsätzlich durch eine Konkretisierung der Rahmenkonzeptprinzipien erfolgen. Um den Zweck der Entscheidungsnützlichkeit zu erfüllen, kann das IASB unter bestimmten Umständen, bspw. aufgrund veränderter konzeptioneller Ansichten oder des ökonomischen Umfelds, ${ }^{203}$ jedoch vom Rahmenkonzept abweichende Regelungen treffen, die dann in der Basis for Conclusions des jeweiligen Standards anzugeben sind. ${ }^{204}$ So stellt z. B. die Regelung in IFRS 15.56 zur Begrenzung der Schätzung variabler Vergütungsbestandteile keine konsequente Umsetzung des im Rahmenkonzept verankerten Vorsichtsprinzips dar. ${ }^{205}$ Diese asymmetrisch vorsichtige Einschränkung auf Beträge, für die höchstwahrscheinlich keine signifikante Stornierung erforderlich ist, soll eine Überbewertung der Umsatzhöhe zugunsten der hierdurch erreichten höheren Entscheidungsnützlichkeit verhindern; ${ }^{206}$ sie entspricht jedoch weder einer am Neutralitätsgrundsatz orientierten Konkretisierung des Vorsichtsprinzips noch der auf Rahmenkonzeptebene erfolgten Abschaffung der Wahrscheinlichkeitsschwellen. ${ }^{207}$

Auf Einzelstandardebene lassen sich zahlreiche weitere induktiv geprägte Regelungen identifizieren. So sind die oben genannten Wesentlichkeitseinschränkungen sowie der Verweis auf die Undurchführbarkeit ${ }^{208}$ der Anwendung einer Rechnungslegungsvorschrift als praxisorientierte Vereinfachungsregelungen $\mathrm{zu}$ werten. Auch die Portfolio-Option in IFRS 15.4 soll als praktischer Behelf die praxisgerechte Anwendbarkeit erleichtern. ${ }^{209}$ Zudem können unterschiedliche Bilanzierungspraktiken besondere Rechnungslegungsvorschriften begründen und von Interessengruppen an das IASB oder das IFRS Interpretations Committee herangetragen werden, ${ }^{210}$ was zu branchenspezifischen Standards führen kann, wie bspw. IFRS 6 Exploration und Evaluierung von Bodenschätzen ${ }^{211}$ und IFRS 17 oder IFRIC 20 Abraumkosten in der Produktionsphase eines Tagebaubergwerks.

\footnotetext{
203 Vgl. CF (2018), BC0.23.

204 Vgl. CF (2018), SP1.3.

${ }^{205} \mathrm{Vgl}$. IFRS 15.BC207 i. V. m. CF (2018), 2.17.

206 Vgl. IFRS 15.BC203-BC207.

207 Vgl. Wagenhofer (2014), S. 370 f. und Note 49.

208 Vgl. IAS 1.BC36.

${ }^{209}$ Vgl. IFRS 15.BC69; ausführlich Abschnitt 3.1.2.3.1.2.

210 Vgl. Due Process Handbook, Rz. 5.15.

${ }^{211}$ Vgl. zum Einfluss der Bilanzierungspraxis Dobler (2014), S. 76 f.
} 
Inwieweit der Konflikt zwischen einer an der Rechnungslegungspraxis orientierten induktiven Normermittlung und der mithilfe des Rahmenkonzepts angestrebten konzeptionell deduktiven Standardsetzung im Standardsetzungsprozess gelöst wird, ist besonders bei einer von den theoretischen Anforderungen abweichenden Bilanzierungspraxis fraglich. Da das Rahmenkonzept neben der Zwecksetzung und den allgemeinen Prinzipien auch Praktikabilitätserwägungen beinhaltet, scheint eine eindeutige Zuordenbarkeit des IFRSStandardsetzungsprozesses $\mathrm{zu}$ einem der beiden Normermittlungsansätze nur bedingt möglich. Anstelle einer einseitigen Charakterisierung, wonach die Standardüberarbeitung bzw. Standardentwicklung entweder das Ergebnis normativer Deduktion oder einzelfallspezifischer Induktion darstellt, wird der Standardsetzungsprozess im folgenden Abschnitt als Prozess wechselseitiger Beeinflussung von IASB und beteiligten Interessengruppen untersucht.

\subsection{Normermittlung unter Zuhilfenahme der Hermeneutik als Erklärungsansatz}

\subsubsection{Kompromisscharakter von Rechnungslegungsvorschriften}

\subsubsection{Standardsetzung als politischer Prozess}

\subsection{Rechnungslegungsregulierung und Legitimationserfordernis}

„Das Rechnungswesen ist eine gesellschaftliche Institution, [...] [bei der es um nicht weniger geht] als die Definition, die rechnerische Konkretisierung sowie die Planung und Kontrolle des Kapitals“212. Als solche hat es weder einen universell gültigen noch abstrakt theoretischen Ursprung, sondern wird vielmehr sozial konstruiert ${ }^{213}$ und durch das kulturelle, ökonomische und politische Umfeld geprägt. ${ }^{214}$ Gleichzeitig wirkt sich die Rechnungslegung durch die Bereitstellung von Informationen auf das jeweilige gesellschaftliche Umfeld aus und führt zu unterschiedlichen Wohlfahrtseffekten, die eine Regulierung begründen. ${ }^{215}$ Die Gestaltung von Rechnungslegungsnormen ist dabei Ergebnis eines staatlichen Eingriffs und/oder der Auseinandersetzung zwischen betroffenen Interessengruppen, deren Wohlfahrt durch Form und Inhalt der Regelungen beeinflusst wird, und

\footnotetext{
212 Ordelheide (1998), S. 2.

${ }^{213}$ Vgl. Alexander/Archer (2003), S. 5 f.; Macintosh (2009), S. 162-168.

214 Vgl. Burchell u. a. (1980), S. 7-11; Rost (1991), S. 80; Chapman u. a. (2009), S. 1-20.

${ }^{215}$ Vgl. Beaver (1989), S. 17 f., 43 f., 177-193; Bromwich/Hopwood (1983), S. x f.
} 
stellt somit nicht nur eine technische Aufgabe, sondern einen politischen Prozess dar. $^{216}$ Während eine staatliche Regulierung eine demokratische Gesetzgebung gewährleistet, ist eine private Regulierung aufgrund der für die Standardsetzung verantwortlichen Experten durch ein hohes Maß an Fachwissen geprägt. ${ }^{217}$ Die Rechnungslegungsregulierung für Konzernabschlüsse kapitalmarktorientierter Unternehmen innerhalb der EU ist als hybride Regulierung zu charakterisieren, ${ }^{218}$ bei der die Standardentwicklung an die IFRS Foundation als privatrechtliche Organisation delegiert und im Rahmen des Endorsement-Prozesses durch den europäischen Gesetzgeber ${ }^{219}$ normiert wird.

Als transnationale, nicht demokratisch legitimierte Institution ${ }^{220}$ ist die Rechtmäßigkeit der IFRS Foundation respektive der vom IASB entwickelten und noch nicht in EU-Recht übernommenen Standards von ihrer normativen Gültigkeit sowie Akzeptanz ${ }^{221}$ abhängig und lässt sich durch input-orientierte, d. h. mitwirkungsorientierte, und output-orientierte, d. h. effizienz- und effektivitätsorientierte, Legitimationsargumente ${ }^{222}$ begründen. $^{223}$ Zwar unterscheidet sich die input-orientierte Perspektive durch den Fokus auf individuelle Mitbestimmungsmöglichkeiten und kollektiv übereinstimmende Entscheidungen von der auf Mehrheitsentscheidungen basierenden und Allgemeinwohl fördernden outputorientierten Perspektive, ,[j]edoch koexistieren [...] input- und output-orientierte Legitimität Seite an Seite, und sie verstärken, ergänzen und ersetzen sich gegenseitig“.224 Sowohl die input-orientierten Komponenten der Partizipation und des Konsens sowie des transparenten und offenen Entscheidungsprozesses als auch die output-orientierte Komponente der markteffizienten Problemlösung 225

${ }^{216}$ Vgl. Burggraaff (1983), S. 4; Cooper/Sherer (1984), S. 208; Sutton (1984), S. 81; Ordelheide (1998), S. 2; Zeff (2007), S. 299-301; André u. a. (2009), S. 9-22.

${ }^{217}$ Vgl. Achleitner (1995), S. 181-208; Kurz (2009), S. 18-21.

218 Vgl. Kirchner/Schmidt (2005), S. 71-73; Kirchner/Schmidt (2006), S. 392-396; ebenso zur hybriden Rechtsfortbildung Kirchner (2005), S. 202 f.

219 Vgl. IAS-Verordnung, Artikel 2-4.

${ }^{220}$ Vgl. Huber (2008), S. 392-396.

${ }^{221} \mathrm{Vgl}$. zum Unterschied zwischen dem normativen und positiven Legitimationsverständnis Homann (1999), S. 53-57.

${ }^{222}$ Vgl. grundlegend zu den beiden Legitimationsperspektiven Scharpf (1970), S. 21-28; Scharpf (2004), S. 5-9.

${ }^{223}$ Vgl. Richardson/Eberlein (2011), S. 219-238.

${ }^{224}$ Vgl. Scharpf (1999), S. 16-22 (auch Zitat, S. 21); zur Input- und Output-Legitimation sowie zur Throughput-Legitimation Fischer (2007), S. 334-338; Tamm Hallström/Boström (2010), S. 140-162.

${ }^{225}$ Vgl. zu den input- und output-orientierten Komponenten Pàllinger (2005), S. 5 f. 
lassen sich in der IFRS-Standardsetzung identifizieren. ${ }^{226}$ So ist die von der EU erhaltene Kompetenz zur Entwicklung kapitalmarktfördernder Rechnungslegungsstandards und der dafür erforderlichen Bündelung von Expertenwissen im IASB sowie die über den europäischen Kapitalmarkt hinausgehende Anwendung der IFRS Ausdruck output-orientierter Legitimation. ${ }^{227}$ Um die Akzeptanz zu gewährleisten, bedarf es jedoch wiederum der Zustimmung der Betroffenen. Durch den als Konsultationsverfahren angelegten offenen Standardsetzungsprozess werden daher „prozedurale Mindestanforderungen“ für den Einbezug betroffener Akteure sowie der Transparenz sichergestellt, die der Förderung der Input-Legitimation dienen. ${ }^{228}$ Trotz der Komplementarität beider Legitimationsformen besteht folglich ein Trade-Off zwischen einer gänzlich output-orientierten, auf die wohlfahrtsökonomische Effizienz des Kapitalmarkts ausgerichteten und einer ausschließlich input-orientierten, den individuellen Nutzen aller Beteiligten berücksichtigenden und auf einen Konsens abzielenden Standardsetzung. ${ }^{229}$ Zur Sicherstellung eines ausgewogenen Legitimationsniveaus dient der Due Process daher der diskursiven Auseinandersetzung, ${ }^{230}$ fraglich ist jedoch inwieweit die sich aus diesem Prozess ergebenden Rechnungslegungsvorschriften einen Konsens oder vielmehr einen - einseitigen oder mehrheitlich tolerierbaren - Kompromiss abbilden. $^{231}$

\subsection{Politische Ökonomie der Rechnungslegung und Lobbying-Anreize}

Aus der politischen Komponente des privaten Standardsetzungsprozesses ergibt sich, dass Rechnungslegungsvorschriften als Resultat andauernder Verhandlungen zwischen Interessengruppen zu betrachten sind. ${ }^{232}$ Besonders die Abgrenzung

\footnotetext{
${ }^{226}$ Vgl. Botzem/Dobusch (2012), S. 749-751; zur Kritik und Ablehnung der Legitimation der IFRS Foundation und des IASB Burlaud/Colasse (2011), S. 28-37.

${ }^{227}$ Vgl. Huber (2008), S. 396; Kurz (2009), S. 48; Botzem (2014), S. 943.

${ }^{228}$ Vgl. Michael (2005), S. 449-454 (auch Zitat, S. 449); Botzem/Quack (2006), S. 282-284; Botzem (2014), S. 943; zur ausführlichen Diskussion der Legitimationsbasis des IASB Kurz (2009), S. 103-151.

${ }^{229}$ Vgl. Botzem/Dobusch (2012), S. 743 f., 752.

${ }^{230}$ Vgl. Richardson (2008), S. 683.

${ }^{231}$ Vgl. Schmitz (2012), S. 216-219.

232 Vgl. Peasnell (1982), S. 248-250; Achleitner (1995), S. 47.
} 
zwischen der wünschenswerten Teilnahme und dem Einbezug aller Interessengruppen sowie der andererseits nur einseitigen Einflussnahme und Berücksichtigung dominierender Gruppen ist häufig jedoch fließend. ${ }^{233}$ Unter dem Forschungsansatz der politischen Ökonomie der Rechnungslegung wird der Entstehungsprozess von Vorschriften daher regulierungstheoretisch untersucht. ${ }^{234}$ Während die normative Theorie der Regulierung eine gesamtwohlsteigernde Regulierungsinstanz unterstellt, gründet die positive Theorie der Regulierung auf den individuellen Motiven der an der Regulierung beteiligten Akteure. ${ }^{235}$ Da durch das (eigennutzorientierte) Einwirken von Interessengruppen auf den Prozess der Rechnungslegungsregulierung grundsätzlich individuell vorteilhafte Regelungen geschaffen werden können, ist die Entwicklung von Regelungen im Sinne der positiven Regulierungsperspektive aus den Aktivitäten der Interessengruppen zu erklären, ,die gezielt in den politischen Prozeß [eingreifen], um Veränderungen institutioneller Rahmenbedingungen herbeizuführen. “236

Als Lobbying wird dabei jegliche Form der Einflussnahme von Interessengruppen auf die Ausgestaltung von Regelungen verstanden, deren Anreiz auf einer individuellen Kosten-Nutzen-Abwägung beruht. ${ }^{237}$ Im Rahmen der Rechnungslegung besteht vor allem bei Unternehmen als Anwender der Regelungen eine hohe Motivation zum Lobbying im Standardsetzungsprozess, insbesondere wenn die vom Standardsetzer vorgeschlagenen Regelungen vom Status quo abweichen und einen höheren Aufwand sowie eine aus Unternehmenssicht unvorteilhaftere Darstellung der Unternehmenslage bewirken. ${ }^{238}$ Sofern einzelne Interessengruppen den Standardsetzer im Sinne der regulierungstheoretischen „capture“-Theorie im Extremfall gänzlich zu ihren Gunsten vereinnahmen können, wird die Standardsetzung zum Produkt individueller Interessenausrichtung. ${ }^{239}$ So birgt eine einseitige Berücksichtigung von Unternehmensinteressen die Gefahr, dass ,die Rechnungslegungspraxis nicht bloß als eine unter vielen Erkenntnisquellen [angesehen], sondern ihr unmittelbare autoritative Wirkung

\footnotetext{
${ }^{233}$ Vgl. Berndt (2005), S. 190.

${ }^{234}$ Vgl. Ordelheide (1998), S. 3-7; Tietz-Weber (2006), S. 41-46; Zülch u. a. (2009), S. 4 f. ${ }^{235}$ Vgl. Feldhoff (1994), S. 529-532; Kurz (2009), S. 9-18; Homfeldt (2013), S. 172 f.

236 Vgl. Wenger (1996), S. 426 (auch Zitat).

${ }^{237}$ Vgl. Sutton (1984), S. 81-83; ausführlich zur Motivation Königsgruber (2009), S. 13121315.

${ }^{238}$ Vgl. etwa Kenny/Larson (1993), S. 536-538; Jorissen u. a. (2012), S. 698-700; zur Motivation anderer Interessengruppen Tietz-Weber (2006), S. 64-66; Auste (2011), S. 22-27.

${ }^{239}$ Vgl. Posner (1974), S. 341 f.; Cortese (2011), S. 405-408.
} 
[zugesprochen wird]،“240 Umgekehrt führt eine gänzlich an den Informationsinteressen der Rechnungslegungsnutzer ausgerichtete Normsetzung zu wenig Akzeptanz bei Unternehmen, wenn die Nutzerinteressen von der gewünschten Bilanzierungspraxis abweichen. ${ }^{241}$

Um die Legitimation entwickelter Standards sicherzustellen, bedarf es daher eines normativen Beurteilungsmaßstabs, der dem IASB eine begründete Interessenabwägung im Sinne einer Kompromissfindung ermöglicht. Diese Funktion wird dem Rahmenkonzept zugeschrieben, das technisch den Prozess der Normentwicklung steuern, gleichwohl unter Legitimationsgesichtspunkten einer interessengeleiteten Einflussnahme entgegenwirken soll. ${ }^{242}$ Das Rahmenkonzept fungiert demnach nicht nur als Deduktions-, sondern auch als Legitimationsgrundlage, indem es die Satzung des IASB als konzeptioneller Bezugspunkt ergänzt, wodurch die Rechtmäßigkeit des Due Process und folglich der in diesem Prozess entwickelten Standards vermittelt werden soll. ${ }^{243}$ Durch die Rechtfertigung einer Standardsetzungsentscheidung auf Basis des Rahmenkonzepts kann insofern die Akzeptanz entwickelter Standards erhöht werden. ${ }^{244}$ Die Standardsetzung kann daher als rekursiver Prozess betrachtet werden, bei dem Rechnungslegungsstandards aus den Wechselwirkungen zwischen dem IASB und den betroffenen Interessengruppen resultieren, wobei die entwickelten Standards als institutioneller Rahmen wiederum auf das Handlungsfeld der einzelnen Akteure einwirken. ${ }^{245}$

\subsubsection{Ermittlung kompromissfähiger Rechnungslegungsvorschriften}

\subsection{Hermeneutik als erkenntnistheoretische Methode}

Zur Interpretation des interaktiven Standardsetzungsprozesses und zum Verständnis der aus dem Prozess resultierenden Regelungen ist eine erkenntnistheoretische Methode heranzuziehen. Die Hermeneutik als „eine Methode der wissenschaftlichen Annäherung an Gegenstände" gilt als disziplinenübergreifendes Verfahren

\footnotetext{
${ }^{240}$ Vgl. Berndt (2005), S. 177 (auch Zitat); zum Beispiel der Öl- und Gasindustrie Cortese (2011), S. 408-416.

${ }^{241}$ Vgl. Berndt (2005), S. 177.

${ }^{242} \mathrm{Vgl}$. Solomons (1983), S. 110-112, 115.

${ }^{243}$ Vgl. Botzem (2010), S. 111; zur Notwendigkeit eines Rahmenkonzepts für die Legitimation eines Standardsetzers Solomons (1983), S. 115; kritisch zur Legitimationsfähigkeit des IASB-Rahmenkonzepts Burlaud/Colasse (2011), S. 31-37.

${ }^{244} \mathrm{Vgl}$. Dennis (2018), S. 388 f.

${ }^{245} \mathrm{Vgl}$. Botzem u. a. (2017), S. 554-557; grundlegend zum ,recursive law-making“ Halliday/Carruthers (2007), S. 1142-1153; Malets/Quack (2017), S. 334-341.
} 
des Sinnverstehens. ${ }^{246}$ Der Verstehensprozess erfolgt dabei rekursiv, d. h. um Texte, wie bspw. Regelungsvorschriften, verstehen zu können, bedarf es eines gewissen Vorverständnisses, das jedoch durch die Deutung des Textes bedingt wird, die sich wiederum auf das Vorverständnis auswirkt. ${ }^{247}$ „Dieses Hin und Her zwischen Vorverständnis und Deutung begründet den hermeneutischen Zir-

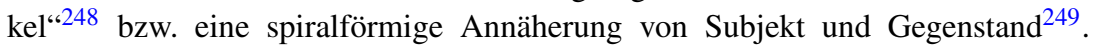
Im Rahmen von Gesetzgebungsverfahren bzw. Standardsetzungsprozessen stehen Norm und Sachverhalt insofern in einem dynamischen Verhältnis, als die Konkretisierung einer Norm unter Berücksichtigung ihrer Anwendung auf einen Sachverhalt erfolgt, der relevante Sachverhalt indes erst mithilfe der Norm konstituiert wird. ${ }^{250}$ Für die Anwendung einer Regelung auf einen Fall bedarf es somit der Auslegung, um den mit der Regelung verbundenen Sinn zu rekonstruieren. ${ }^{251}$ Die Hermeneutik als erkenntnistheoretische Methode ist daher gleichsam eine Auslegungsmethode im Rahmen der Gesetzes- bzw. Standardanwendung. So ist in der juristischen Hermeneutik ${ }^{252}$ der ,fachmännische Umgang [...] mit dem Gesetz" an den Auslegungskanon ${ }^{253}$ aus Wortsinn, Entstehungsgeschichte, Systematik und Telos sowie die Argumentationslehre geknüpft, die ,,als Entscheidungsregeln [...] die Verbindung zwischen Norm und Sachverhaltsentscheidung herstellen und sichern [s]ollen" sowie den Auslegungsspielraum strukturieren und getroffene Entscheidungen zu begründen vermögen. 254

\subsection{Rekursives Verhältnis zwischen Standardsetzung und Standardanwendung}

In der IFRS-Standardsetzung spiegelt sich der rekursive Verstehensprozess in der kontinuierlichen Rückkoppelung zwischen Standardsetzungsprozess und Regelungsumsetzung durch die betroffenen Akteure wider. Entsprechend dem

\footnotetext{
${ }^{246}$ Vgl. Hassemer (1986), S. 195 f. (auch Zitat, S. 195).

${ }^{247}$ Vgl. Gadamer (2010), S. 271; Hassemer (1986), S. 208; Larenz (1991), S. 206-211; Röhl/Röhl (2008), S. 116 f.

${ }^{248}$ Röhl/Röhl (2008), S. 117.

${ }^{249}$ Vgl. Hassemer (1986), S. 208; Larenz (1991), S. 206 f.

${ }^{250}$ Vgl. Hassemer (1986), S. 201.

251 Vgl. Röhl/Röhl (2008), S. 117.

${ }^{252}$ Vgl. zur Abgrenzung der allgemeinen und juristischen Hermeneutik Nerlich (2007), S. 32-36 m. w. N.

${ }^{253}$ Vgl. Larenz (1991), S. 316-343; Röhl/Röhl (2008), S. 613-623; zur Anwendbarkeit des Auslegungskanons bei der Auslegung der IFRS Abschnitt 2.2.2.2.

${ }^{254}$ Vgl. Hassemer (1986), S. 203 f. (auch Zitate, S. 203).
} 
strukturationstheoretischen Ansatz tragen Regelungen zur Struktur eines rekursiven sozialen Systems bei, das durch Akteure genutzt und durch ihr Handeln reproduziert wird. ${ }^{255}$ Es bestehet dabei ein rekursiver Zusammenhang zwischen den Dimensionen der Signifikation, Legitimation und Herrschaft: ${ }^{256}$ Das Rechnungswesen ist als Bündel interpretativer Schemata zu verstehen, die dem Verständnis von Bilanzierungssachverhalten dienen und durch das Verständnis der Bilanzierungspraxis gleichzeitig bedingt werden; sie entfalten eine normative Bindungswirkung und werden durch über unterschiedliche Ressourcen verfügende Akteure beeinflusst. ${ }^{257}$ Akteure, die von den Regelungen betroffen sind, regulieren bzw. gestalten das sie regulierende institutionelle Umfeld demzufolge selbst, ${ }^{258}$ wodurch die Standardsetzung zum ,Medium und [...] Produkt [...] strategischen Handelns" wird. ${ }^{259}$ Dabei wird der Output der einzelnen Akteure zum Input des Prozesses, dessen Output - neue oder veränderte Regelungen - erneut zum Input der Akteure wird. 260

Diese wechselseitige Abhängigkeit besteht insbesondere bei transnationalen Institutionen, ${ }^{261}$ wie auch der IFRS Foundation respektive den IFRS, da die Regelungen von der Legitimation und damit der Anwendung durch die ,räumlich und funktional verteilte[n]“ Regulierten abhängen. ${ }^{262}$ Es besteht insofern ein rekursives Verhältnis zwischen „law in action“ und „law on the books“, als kodifizierte Regelungen im Rahmen ihrer Anwendung interpretiert und modifiziert werden und ihre Anwendung wiederum auf die Formulierung und Änderung kodifizierter Regelungen einwirkt. ${ }^{263}$ Die Standardsetzung ist demnach durch „vielfache[.] Phasen der Innovation, Standardisierung und Normierung" gekennzeichnet, in

${ }^{255}$ Vgl. grundlegend zur Theorie der Strukturierung Giddens (1997), S. 51-90; mit Bezug zur Rechnungslegung Alexander/Archer (2003), S. 10.

${ }^{256}$ Vgl. Giddens (1997), S. 81-88; Dillard u. a. (2004), S. 513-522; Ortmann/Zimmer (2001), S. 313 f.

${ }^{257}$ Vgl. Roberts/Scapens (1985), S. 448 f.; Guerreiro u. a. (2014), S. 383.

${ }^{258}$ Vgl. zur Endogenisierung des Rechts Edelman u. a. (1999), S. 407; Edelman (2016), S. 27-41; Bozanic u. a. (2012), S. 464-478.

${ }^{259}$ Vgl. Ortmann u. a. (2000), S. 327 f. (auch Zitat, S. 328).

${ }^{260}$ Vgl. Ortmann/Zimmer (2001), S. 314.

261 Vgl. Halliday/Carruthers (2007), S. 1146; Ortmann/Zimmer (2001), S. 310 f.

${ }^{262}$ Vgl. Quack (2009), S. 577 (auch Zitat); Botzem/Dobusch (2012), S. 752-756.

${ }^{263}$ Vgl. Halliday/Carruthers (1998), S. 45-62 (auch Zitat, S. 46); Halliday/Carruthers (2007), S. 1146-1148; zum rekursiven Zusammenhang zwischen Standardentstehung und -verbreitung Botzem/Dobusch (2012), S. 755-757. 
denen sich unterschiedliche Akteure beteiligen. ${ }^{264}$ Im Rahmen der Innovationsphase werden die kodifizierten Regelungen durch einzelne Akteure angewendet, wobei der Regelungsinhalt durch die Anwendung - besonders bei Mehrdeutigkeit des Regelungswortlauts oder Regelungslücken - interpretiert, umgangen oder reformiert wird. ${ }^{265}$ In der Phase der Standardisierung erfolgt eine Zusammenfassung und Vereinheitlichung der sich aus der Anwendung entwickelten Praktiken durch private Organisationen oder die faktische Befolgung etablierter Standardpraktiken, die in der Phase der Normierung durch rechtliche Anerkennung verbindlich kodifiziert werden. ${ }^{266}$ Einer Änderung normierter bzw. der Kodifizierung neuer Regelungen geht in der Regel ein ,triggering event“ voraus, weshalb trotz Veränderungen in der Anwendung der Regelungen die Normsetzung über einen längeren Zeitraum ausstehen kann. ${ }^{267}$ Innerhalb und zwischen den einzelnen Phasen bestehen dabei Feedback-Schleifen, in denen einzelne Akteure in ihren unterschiedlichen Funktionen als Innovatoren, Intermediäre und Lobbyisten Einfluss nehmen. ${ }^{268}$

Auch die IFRS-Standardsetzung ist durch diese rekursiven Beziehungen geprägt, wobei der Due Process als ,channel for feedback“ dient. ${ }^{269}$ Die vom IASB erlassenen und von der EU normierten Standards ${ }^{270}$ werden von Unternehmen und anderen Akteuren angewendet. Bei Anwendungsspielräumen oder Regelungslücken wird der Regelungsinhalt von den Anwendern, besonders den Unternehmen als Abschlussersteller, interpretiert und eine Bilanzierungslösung entwickelt, die sich branchenweit oder -übergreifend ausdehnen kann. ${ }^{271}$ Defizite eines Rechnungslegungsstandards können wiederum eine Standardüberarbeitung bzw. -anpassung durch das IASB begründen, ${ }^{272}$ wie bspw. die Entwicklung des IFRS 15, der bestehende Inkonsistenzen zwischen den alten Standards und die bis dahin durch die Bilanzierungspraxis ${ }^{273}$ im Rahmen der Anwendung

\footnotetext{
264 Vgl. Quack (2009), S. 577 (auch Zitat); Quack (2007), S. 652.

${ }^{265}$ Vgl. Halliday/Carruthers (1998), S. 58-62; Halliday/Carruthers (2007), S. 1148 f.; Quack (2009), S. 589-592.

${ }^{266}$ Vgl. Quack (2009), S. 592-597.

${ }^{267}$ Vgl. Halliday/Carruthers (2007), S. 1146-1148 (auch Zitat, S. 1147).

${ }^{268}$ Vgl. Quack (2009), S. 588 f.

${ }^{269}$ Vgl. Botzem u. a. (2017), S. 556 (auch Zitat).

${ }^{270} \mathrm{Vgl}$. zum Ursprung der IFRS als Zusammenfassung nationaler Bilanzierungspraktiken, Thorell/Whittington (1994) S. 224.

${ }^{271}$ Vgl. zur Auslegung und Schließung von Regelungslücken Abschnitt 2.2.2.2.

${ }^{272}$ Vgl. Abschnitt 2.2.2.1.1 und 2.2.2.1.2.

${ }^{273}$ Vgl. zur Anwendung der US-GAAP Regelungen in der Praxis zur Bilanzierung von Mehrkomponentengeschäften Fürwentsches (2010), S. 134-136, 182 f.
} 
geschlossene Regelungslücke durch einheitliche Regelungen beseitigen sollte. ${ }^{274}$ Im Rahmen des Due Process werden Standardentwürfe veröffentlicht, die von unterschiedlichen Akteuren kommentiert werden und deren Feedback dem IASB als Informationsgrundlage dient. ${ }^{275}$ Die Anfertigung einer Stellungnahme verlangt dabei eine Auseinandersetzung mit den Anwendungsmöglichkeiten vorgeschlagener Rechnungslegungsvorschriften, sodass bereits im Due Process eine Normauslegung stattfindet, die in Abhängigkeit der Berücksichtigung durch das IASB in die weitere Standardentwicklung einfließen kann. Das IASB interpretiert wiederum die Stellungnahmen und legt die vorgeschlagenen Regelungen im Lichte dieser Erkenntnisse erneut aus. Der Due Process verläuft daher in mehreren Feedback-Phasen, in denen die Meinungen der am Prozess Beteiligten zusammengefasst und die Standardentwürfe umformuliert bzw. angepasst werden sowie erneut Feedback eingeholt wird. ${ }^{276}$ Der finale Standard wird letztlich von der EU übernommen und ist wiederum von den betroffenen Unternehmen und anderen Akteuren anzuwenden. Standardanwendung und Standardsetzung bedingen sich gegenseitig; die IFRS-Standardsetzung beruht als ,interactive process of meaning making" folglich auf der Interaktion zwischen dem IASB und den Interessengruppen. ${ }^{277}$

\subsection{Implikationen für den IFRS-Standardsetzungsprozess}

Unter Zuhilfenahme der Hermeneutik ${ }^{278}$ werden die Elemente der deduktiven Normermittlung, bei der die Konkretisierung von Einzelnormen aus übergeordneten Prinzipien von oben an die Sache erfolgt, und die der induktiven Normermittlung, bei der die Verallgemeinerung etablierter Bilanzierungspraktiken von unten auf eine Rechtsnorm abzielt, vereint, sodass bei der Normfindung „Norm und Fall [...] schrittweise zueinander in eine Beziehung gebracht werden [und] konkretisierend aufeinander hin [...] entwickel[t] [werden] ${ }^{\text {“279 }}$. Einzelnormen werden demzufolge ausgehend vom Rahmenkonzept als theoretischen Maßstab in Einklang mit den dort festgelegten Grundprinzipien deduktiv erschlossen (,forward argument"), wohingegen sich ausgehend von der Bilanzierungspraxis

${ }^{274} \mathrm{Vgl}$. IFRS 15.IN4 f.

${ }^{275}$ Vgl. zur Diskussion des inhaltlichen Erkenntnisgewinns Botzem (2010), S. 143-146.

276 Vgl. Botzem u. a. (2017), S. 556.

${ }^{277}$ Vgl. Cortese u. a. (2010), S. 77-80 (auch Zitat, S. 78), bezugnehmend auf Fairclough (2003), S. 10-12; ebenso Røsok (2018), S. 10.

${ }^{278}$ Vgl. zur hermeneutischen Methode in der Rechnungslegung Schulte (2010), S. 79-81; Christ (2014), S. 67-74; Müller (2015), S. 753.

${ }^{279}$ Vgl. grundlegend zur juristischen Hermeneutik Hassemer (1986), S. 195-212 (auch Zitat, S. 201); Röhl/Röhl (2008), S. 116-122. 
diesen Einzelnormen induktiv genähert wird (,,backward argument“). ${ }^{280}$ Das Rahmenkonzept als auch die Bilanzierungspraxis beruhen dabei auf Konventionen bzw. Rechnungslegungsannahmen, ${ }^{281}$ wobei das Rahmenkonzept als „eine Art reflektiertes „Vorverständnis“ des Normsetzers“ aufgefasst werden kann. ${ }^{282}$ Es dient weniger der systematischen und logischen Ableitung von Einzelnormen, sondern legt vielmehr einzelne Grundprinzipien fest, die den der Standardsetzung immanenten Kommunikationsprozess strukturieren sollen und insoweit einen normativen Rahmen vorgeben. ${ }^{283}$ So gelten die im Rahmenkonzept kodifizierten Ansatzkriterien bspw. nicht als „overarching principle“, sondern sollen als „guidance“ bzw. „tools“ den Entscheidungsprozess auf Einzelstandardebene unterstützen. ${ }^{284}$ Die Bilanzierungspraxis deutet eine Regelungsvorschrift auf Basis ihres Vorverständnisses, das auf grundlegenden Annahmen und Kenntnissen aus der Anwendung beruht, gleichzeitig jedoch vom Rahmenkonzept geprägt und wiederum durch die Auslegung der Regelung beeinflusst wird.

Bei der Ermittlung der Rechnungslegungsvorschriften konvergieren die auf dem Rahmenkonzept basierenden und die aus der Anwendung resultierenden Überlegungen; das Ergebnis ist ein sich gegenseitig bedingender Kompromiss. ${ }^{285}$ Dies wird auch am Beispiel des IFRS 15 deutlich, bei dem die Entwicklung der Einzelregelungen unter Berücksichtigung des Zwecks der Entscheidungsnützlichkeit und des Ansatzkriteriums der Kontrolle erfolgte, aufgrund der in Praxis sachgerechten, unterschiedlichen Umsetzungsmöglichkeiten des Kontrollübergangs jedoch anstelle des angestrebten Asset-Liability-Ansatzes in einer die Darstellung der Unternehmenslage konkretisierenden Lösung mündete. ${ }^{286}$ Inwieweit die aus dem Standardsetzungsprozess resultierenden Rechnungslegungsvorschriften als richtig angesehen bzw. akzeptiert werden, ist wesentlich vom Standardsetzungsverfahren abhängig, das den „Verstehensprozess“ aufdecken und

\footnotetext{
${ }^{280}$ Vgl. Power (1993), S. 54-56 (auch Zitat, S. 56); Archer (1997), S. 237-239; grundlegend zur Idee des ,reflective equilibrium“ Rawls (1993), S. 1-220.

${ }^{281}$ Vgl. Power (1993), S. 55 f.

${ }^{282}$ Vgl. zum Rahmenkonzept des FASB Wüstemann (1999), S. 125 f. (auch Zitat).

${ }^{283}$ Vgl. Power (1993), S. 55 f.; Macve (1997), S. xxi f.; zum Rahmenkonzept als Satzung der Standardsetzung Christensen (2010), S. 297.

${ }^{284}$ Vgl. CF (2018), BC5.6 und BC5.10 (auch Zitate); Due Process Handbook, Rz. 4.20.

${ }^{285}$ Vgl. Power (1993), S. 50; zur Notwendigkeit des Kompromisses auf Einzelstandardebene Macve (1997), S. xxi f.

${ }^{286}$ Vgl. Wagenhofer (2014), S. 366-368.
} 
eine reflektierte Begründung liefern sollte. ${ }^{287} \mathrm{Um}$ im Standardsetzungsprozess einen Kompromiss und damit die Legitimation der entwickelten Regelungen zu erreichen, bedarf es daher eines diskursiven Standardsetzungsverfahrens.

\subsubsection{Due Process als diskursiver Normermittlungsprozess}

\subsubsection{Entwicklung von Rechnungslegungsvorschriften im Rahmen der Standardsetzung}

\subsection{Standardsetzung durch das IASB}

\subsection{Agendasetzung}

Im Rahmen der IFRS-Standardsetzung dient der Due Process der Sicherstellung einer Entscheidungsfindung auf Basis einer diskursiven Auseinandersetzung. ${ }^{288}$ Gemäß der Satzung der IFRS Foundation, die die Zusammensetzung und den Aufgabenbereich der einzelnen Gremien festlegt, obliegt die Standardsetzungskompetenz primär dem IASB und ergänzend dem IFRS Interpretations Committee. ${ }^{289}$ Im Due Process Handbook sind die prozeduralen Anforderungen des Due Process kodifiziert, deren Einhaltung vom Due Process Oversight Committee überwacht wird und durch Veröffentlichung eines Technical Staff Reports durch das IASB bei jedem Projekt nachzuweisen ist. ${ }^{290}$ Die Verfahrensanforderungen beruhen auf den Prinzipien der Transparenz, der vollständigen und angemessenen Konsultation und der Rechenschaft, wonach in einem transparenten Prozess die Sichtweisen der Beteiligten zu berücksichtigen sowie potenzielle Effekte der Standardsetzung für Betroffene zu analysieren und die getroffenen Entscheidungen zu begründen sind. ${ }^{291}$

Die Standardsetzungsprojekte sind in einem Arbeitsplan, der sog. Technical Agenda, zusammengefasst, die alle fünf Jahre auf Basis der durch öffentliche Konsultation erlangten Informationen festgelegt bzw. überarbeitet wird. ${ }^{292}$ Die Themenauswahl und Prioritätensetzung erfolgt nicht anhand festgelegter Kriterien, unterliegt jedoch entsprechend der nutzerorientierten Zielsetzung

\footnotetext{
${ }^{287}$ Vgl. zur Verifizierung von Sachverhaltsentscheidungen im Rahmen juristischer Hermeneutik Hassemer (1986), S. 210 f. (auch Zitat, S. 210); ausführlich Hassemer (1967), S. 127148.

288 Vgl. Due Process Handbook, Rz. 1.2.

289 Vgl. IFRS Constitution, Rz. 36, 42.

${ }^{290}$ Vgl. Due Process Handbook, Rz. 1.2-1.7, 2.1-2.15.

${ }^{291}$ Vgl. Due Process Handbook, Rz. 3.1.

292 Vgl. Due Process Handbook, Rz. 4.3.
} 
dem Anspruch, die Vergleichbarkeit der Bilanzierungspraxis ${ }^{293}$ und die Konsistenz der Rechnungslegungsvorschriften zu erhöhen sowie Anwendungsprobleme $\mathrm{zu}$ beseitigen und Veränderungen der rechtlichen Rahmenbedingungen bzw. Geschäftspraktiken abzubilden. ${ }^{294}$ Die Festlegung von Themen in der Agenda bedingt weder ihre zeitnahe Bearbeitung noch ihre Umsetzung oder Finalisierung. Einzelne Projekte können vielmehr aufgrund begrenzter finanzieller und personeller Kapazitäten sowie veränderter Prioritäten oder anderer Projekte im Laufe des Due Process verzögert oder (vorübergehend) stillgelegt werden. ${ }^{295}$ So verfolgte das IASB mit der Teilveröffentlichung des Rahmenkonzepts in 2010 eine phasenweise Überarbeitung und verzögerte zunächst die Ausarbeitung ausstehender Rahmenkonzeptteile aufgrund anderer Prioritäten; die Weiterführung und Finalisierung des Rahmenkonzeptprojekts wurde erst im Rahmen der Agenda Konsultation von 2011 wieder als Tätigkeitsschwerpunkt forciert. ${ }^{296}$

Die Agendasetzung bietet eine Basis zur Aushandlung relevanter Standardsetzungsprojekte und der Priorisierung dieser, ${ }^{297}$ wobei der Entscheidungsprozess zunächst auf Ebene des Standardsetzers ${ }^{298}$, mithin zwischen dem IASB und den standardsetzungsbefugten Akteuren ${ }^{299}$ sowie im Fall von Konvergenzprojekten dem $\mathrm{FASB}^{300}$, verläuft. Der öffentliche Konsultationsprozess soll dabei einer praxisirrelevanten ${ }^{301}$ sowie ausschließlich politisch motivierten Themenfestlegung entgegenwirken. ${ }^{302}$ Um den Standardsetzungsprozess „evidence-based“ zu gestalten, sollen sowohl die Wissenschaft als auch die Bilanzierungspraxis als Informationsquelle einbezogen werden, ${ }^{303}$ was Interessengruppen bereits

\footnotetext{
293 Vgl. Young (1994), S. 86 f.

${ }^{294}$ Vgl. Howieson (2009), S. 591 f.; zum FASB Beresford (1988), S. 1-3; Jiang u. a. (2018), S. $4 \mathrm{f}$.

${ }^{295}$ Vgl. Dick/Walton (2007), S. 11; zum FASB und weiteren Gründen Jiang u. a. (2018), S. 5. ${ }^{296}$ Vgl. Technical Staff (2012a), Agenda Paper 14, Rz. 2-7; IASB (2012), S. 33-37.

${ }^{297}$ Vgl. Howieson (2009), S. 580; Birt u. a. (2016), S. 272.

298 Vgl. Young (1994), S. 89-105.

${ }^{299} \mathrm{Vgl}$. zum wesentlichen Einfluss einzelner IASB-Mitglieder und bestimmter Gremien auf die Agendasetzung Ram/Newberry (2017), S. 497-503.

${ }^{300}$ Vgl. Pelger (2016), S. 55-68.

301 Vgl. van Riper (1994), S. 104.

${ }^{302} \mathrm{Vgl}$. Hoogervorst (2012b), S. 2; zum Einbezug unterschiedlicher Rechtsordnungen Camfferman/Zeff (2018), S. 18 f.; zum Einfluss externer Faktoren auf die Agendasetzung Johnson/Swieringa (1996), S. 162-166; Watts (2006), S. 56 f.; Gipper u. a. (2013), S. 529 f. ${ }^{303}$ Vgl. IASB (2012), S. 6-16 (auch Zitat, S. 16).
} 
frühzeitig die Möglichkeit zur Einflussnahme bietet ${ }^{304}$. Die Ergebnisse der Konsultation sollen dem IASB zwar inhaltliche Erkenntnisse liefern, sie haben jedoch nicht zwingend Einfluss auf die Agendasetzung ${ }^{305}$ und verändern meist nicht die bereits vom IASB verfolgten oder von den Beratungsgremien empfohlenen Themenschwerpunkte, weshalb dieses Vorgehen vor allem der Legitimation des Standardsetzungsprozesses dient, indem es einen Austausch zwischen dem IASB und den Interessengruppen fördert. ${ }^{306}$

Sofern sich zwischen den Agendakonsultationen einzelne Umstände ändern oder die Ergebnisse eines Post-Implementation-Reviews Anlass geben, kann das IASB kleinere Standardanpassungen jederzeit sowie größere Standardsetzungsprojekte und die Standardsetzungsprioritäten nach Absprache mit dem Advisory Council und dem Accounting Standards Advisory Forum auf die Agenda setzen bzw. diese anpassen. ${ }^{307}$ Einzelne Themen werden häufig auch vom IFRS Interpretations Committee sowie vom Staff, wie etwa im Fall der regulatorischen Änderungen in Form der geplanten IBOR-Zinssatzreform, ${ }^{308}$ oder von verschiedenen anderen Gremien der IFRS Foundation und anderen Interessengruppen an das IASB herangetragen. ${ }^{309}$ Änderungen des Rahmenkonzepts führen nicht automatisch zu neuen Agendaprojekten; die Notwendigkeit von Standardanpassungen zur Beseitigung von Inkonsistenzen ist im Rahmen der Agendasetzung mit anderen Standardsetzungsprioritäten abzuwägen. ${ }^{310}$ Nach welchem Maßstab das IASB eine Priorisierung vornimmt, ist im Einzelfall jedoch nur begrenzt nachvollziehbar. So wurde z. B. die auf Rahmenkonzeptebene geänderte Vermögensgegenstands- und Schuldendefinition nicht in IAS 37 und IAS 38 Immaterielle Vermögenswerte übernommen, ${ }^{311}$ hingegen ein Projekt zur Aufnahme einer Ausnahmeregelung für IFRS 3 Unternehmenszusammenschlüsse zur Verpflichtungsidentifizierung bei Unternehmenszusammenschlüssen auf die Agenda gesetzt ${ }^{312}$. Zwar gewährleistet dieses Vorgehen eine ressourcenorientierte Themenfestlegung, es führt jedoch im Einzelfall - wie etwa der fehlenden

\footnotetext{
304 Vgl. Sutton (1984), S. 89; indes zur Wahrnehmung der Agendasetzung durch Unternehmen als ineffektive Phase Georgiou (2004), S. 226-228.

305 Vgl. Allen (2018), S. 21-24.

${ }^{306}$ Vgl. Pelger/Spieß (2016), S. 83; Birt u. a. (2016), S. 270 f.; Howieson (2009), S. 580.

${ }^{307}$ Vgl. Due Process Handbook, Rz. 4.3-4.7.

${ }^{308}$ Vgl. Technical Staff (2018a), Agenda Paper 19, Rz. 16-20.

309 Vgl. Due Process Handbook, Rz. 4.10.

${ }^{310}$ Vgl. Due Process Handbook, Rz. 4.23.

311 Vgl. IASB (2018a), S. 17.

${ }^{312}$ Vgl. Technical Staff (2018b), Agenda Paper 10, Rz. 5-7.
} 
Angleichung von Einzelstandards und Rahmenkonzept trotz der Bestrebung einer auf dem Rahmenkonzept basierenden konsistenten Standardsetzung - zu konzeptionellen Widersprüchen.

\subsection{Forschungsphase und Standardsetzung}

Die Standardsetzungsaktivitäten des IASB sind in die drei Projektphasen der Forschung, der Standardsetzung sowie der Instandhaltung und Implementierung unterteilt: Im Rahmen von Forschungsprojekten werden einzelne Problemfelder untersucht, woraufhin über die Aufnahme eines Standardsetzungsprojekts zur Entwicklung neuer oder wesentlichen Änderung bestehender Standards entschieden oder ein Projekt für kleinere Anpassungen implementierter Standards aufgenommen wird. ${ }^{313}$ Die Forschungsphase dient zunächst der Identifizierung von Problemen und Mängeln bestehender Regelungen sowie der Beurteilung möglicher Verbesserungen und soll zudem allgemeinere Entwicklungen in der Finanzberichterstattung thematisieren. ${ }^{314}$ Die Ergebnisse und möglichen Lösungsansätze werden in einem Research Paper und/oder Discussion Paper zusammengefasst sowie Stellungnahmen eingeholt auf Basis derer das IASB unter Abwägung der Kosten und Nutzen über die Aufnahme eines Standardsetzungsprojekts entscheidet; der Nutzen ist dabei anhand bestehender Defizite in der Bilanzierung bestimmter Geschäftsvorfälle, der Relevanz für den Nutzer sowie dem Ausmaß betroffener Unternehmen und Jurisdiktionen zu beurteilen. ${ }^{315}$ So wurde bspw. eine mögliche Anpassung von IAS 21 Auswirkungen von Wechselkursänderungen in Bezug auf langfristig monetäre Posten im Rahmen eines Forschungsprojekts untersucht, aufgrund der hohen Kosten einer Anpassung bei gleichzeitig geringem Nutzen jedoch kein Standardsetzungsprojekt eingeleitet. ${ }^{316}$ Die Forschungsergebnisse zur Überarbeitung von IFRS 6 resultierten zwar 2010 in der Veröffentlichung eines Discussion Paper, das Standardsetzungsprojekt wurde indes aufgrund fehlender Dringlichkeit auf unbestimmte Zeit verschoben. ${ }^{317}$

Sofern die Entwicklung eines neuen oder wesentliche Änderung eines bestehenden Standards beschlossen wurde, erfolgt der Standardsetzungsprozess unter

\footnotetext{
313 Vgl. Due Process Handbook, Rz. 4.8-4.19 i. V. m. 5.1.

314 Vgl. Due Process Handbook, Rz. 4.8 f.

315 Vgl. Due Process Handbook, Rz. 5.4.

${ }^{316}$ Vgl. Technical Staff (2014a), Agenda Paper 8A(a), Rz. 33-35; IASB (2014), S. 2.

317 Vgl. Technical Staff (2016b), Agenda Paper 24D, Rz. 35 f.; Technical Staff (2020), Agenda Paper 19, Rz. 3-12.
} 
Veröffentlichung eines Exposure Drafts, der ausgehend von den Rechercheergebnissen und den Stellungnahmen zum Discussion Paper sowie den Vorschlägen aus den Konsultationen mit unterschiedlichen Gremien und Interessengruppen neben den Regelungsvorschlägen eine Begründung für den jeweiligen Standardentwurf beinhaltet und mithilfe derer das IASB die Öffentlichkeit zur Kommentierung auffordert. ${ }^{318}$ Vor dem Hintergrund der eingegangenen Stellungnahmen und sonstiger Konsultationen sowie möglicher Feldstudien und öffentlicher Anhörungen wird der finale Standard formuliert. ${ }^{319}$ Aufgrund veränderter Prioritäten und anderer Standardsetzungsprojekte sowie etwaiger nicht zu vereinbarender Differenzen zwischen den am Standardsetzungsprozess Beteiligten kann die Finalisierung eines Standards jedoch verschoben oder sogar abgebrochen werden. So wurde z. B. die Überarbeitung von IAS 37 trotz veröffentlichter Exposure Drafts bis zur Fertigstellung des Rahmenkonzepts ausgesetzt, ${ }^{320}$ in diesem Fall jedoch nach mehrjähriger Unterbrechung als Forschungsprojekt wieder aufgenommen. ${ }^{321}$ Sofern am Exposure Draft noch wesentliche Änderungen vorgenommen werden müssen, die vorweg nicht adressiert wurden und folglich nicht kommentiert werden konnten, sind ein weiterer Standardentwurf zu veröffentlichen und Stellungnahmen einzuholen, bevor der Standard finalisiert werden kann. ${ }^{322}$

Der öffentliche Standardsetzungsprozess ist unter Legitimationsgesichtspunkten durchaus effektiv, jedoch besonders bei umfangreichen Standardsetzungsprojekten - verstärkt durch mögliche Prioritätenänderungen des IASB - häufig sehr langwierig und dadurch in seiner Effizienz begrenzt. ${ }^{323}$ Der Projektumfang sowie die -komplexität wirken sich daher wesentlich auf die Dauer eines Standardsetzungsprozesses aus. ${ }^{324}$ Besonders bei kontroversen Themen sowie umfangreichen Standardsetzungsprojekten mit weitreichenden bilanzierungspraktischen Auswirkungen wird der Ausgang eines Projekts von der Kompromissfähigkeit vorgeschlagener Lösungsansätze bestimmt. So verzögerte sich bspw. der Standardsetzungsprozess von IFRS $15 \mathrm{zu}$ Beginn des Projekts aufgrund des

\footnotetext{
318 Vgl. Due Process Handbook, Rz. 6.1-6.5.

${ }^{319}$ Vgl. Due Process Handbook, Rz. 6.19-6.24.

${ }^{320}$ Vgl. zum Einfluss und den Differenzen der am Standardsetzungsprozess Beteiligten Morley (2016), S. 226 f., 231-249.

${ }^{321}$ Vgl. IASB (2016a), S. 30; Technical Staff (2018c), Agenda Paper 22, Rz. 1 f., 7.

${ }^{322}$ Vgl. Due Process Handbook, Rz. 6.25-6.29.

323 Vgl. Maier (2008), S. 79.

${ }^{324}$ Vgl. Gipper u. a. (2013), S. 528.
} 
Dissens zwischen den Board-Mitgliedern im Hinblick auf den zu verwendenden Bilanzierungsansatz. ${ }^{325}$ Zudem erforderte die Finalisierung von IFRS 15 die Veröffentlichung eines Re-Exposure-Drafts, mit dem das IASB formal die in den Stellungnahmen zum Exposure Draft hervorgebrachte Kritik adressierte und vorgenommene Änderungen mit dem Erfordernis der einheitlichen Anwendung des Standards begründete, der faktisch jedoch praxisgerechte Zugeständnisse zugunsten kompromissfähiger Rechnungslegungsvorschriften beinhaltete. ${ }^{326}$

\subsection{Instandhaltungs- und Implementierungsphase}

Die Aufnahme von Nachbesserungsprojekten auf die Agenda zur Anpassung oder Klarstellung implementierter Standards erfolgt im Fall von Anwendungsfragen, die vom IFRS Interpretations Committee und von anderen beratenden Gremien an das IASB weitergeleitet oder bei Post-Implementation-Reviews identifiziert werden, sowie im Rahmen von jährlichen Verbesserungen, die sprachliche Klarstellungen und kleinere Korrekturen umfassen. ${ }^{327}$ Die vorgeschlagenen Änderungen werden in einem Exposure Draft veröffentlicht, der von der Öffentlichkeit kommentiert werden kann. ${ }^{328}$ Standardklarstellungen dienen der Konsistenz bestehender Standards; sie führen nicht $\mathrm{zu}$ neuen oder zur Änderung bestehender Regelungen, sondern nur zum Austausch uneindeutiger Formulierungen oder zur Ergänzung von Leitlinien. ${ }^{329}$ So wurde bspw. in IFRS 3 im Zuge der jährlichen Verbesserung ein erläuternder Absatz zur Anwendung der Erwerbsmethode bei schrittweisen Unternehmenszusammenschlüssen ergänzt oder die Regelung in IAS 23 Fremdkapitalkosten zur Nichteinrechnung bestimmter Fremdkapitalkosten umformuliert. ${ }^{330}$ Mithilfe kleinerer Standardänderungen werden zudem geringfügige Ungenauigkeiten innerhalb und Widersprüche zwischen Standards korrigiert, ${ }^{331}$ wie etwa die Aufhebung des Verweises zur Verwendung des Vorsteuerzinssatzes in IAS 41 Landwirtschaft zur Vereinheitlichung der Fair-Value-Bewertung. ${ }^{332}$

\footnotetext{
325 Vgl. Dick/Walton (2007), S. 14; Baudot (2018), S. 677-688.

${ }^{326}$ Vgl. Re-ED BC (2011), BC12-BC14; zu den praxisgerechten Änderungen am Beispiel des zeitraumbezogenen Kontrollübergangs bspw. Baetge/Celik (2014), S. 365-367.

${ }^{327}$ Vgl. Due Process Handbook, Rz. 5.13-5.19 und Rz. 6.10-6.15; zur Übersicht der Anpassungen in 2020 Große (2020a), S. 301-304; Große (2020b), S. 364 f.

328 Vgl. Due Process Handbook, Rz. 6.1, 6.3, 6.10.

${ }^{329}$ Vgl. Due Process Handbook, Rz. 6.12.

330 Vgl. IASB (2017), S. 7 f., 15 f.

331 Vgl. Due Process Handbook, Rz. 6.13.

${ }^{332}$ Vgl. IASB (2020), S. 15-17.
} 
Im Rahmen der Post-Implementation-Reviews überprüft das IASB die Anwendung neuer oder wesentlich veränderter Standards sowie Änderungen im Bereich der Rechnungslegung und regulatorischer Bedingungen. ${ }^{333}$ Die implementierten Standards werden ca. zwei Jahre nach ihrer Einführung im Hinblick auf die Erfüllung des jeweiligen Standardsetzungsziels beurteilt, wobei Anwendungsprobleme oder unerwartete Kosten identifiziert und mögliche Nachbesserungen diskutiert werden. ${ }^{334}$ Die Durchführung von Post-Implementation-Reviews bezweckt folglich nicht nur eine normative, sondern vielmehr auch praktikabilitätsorientierte Prüfung eines Standards. ${ }^{335}$ Die Beurteilung basiert primär auf öffentlichen Konsultationen, wobei zusätzlich Jahresabschlussanalysen und Umfragen oder Interviews durchgeführt sowie anwendungsrelevante Forschungsbeiträge einbezogen werden können. ${ }^{336}$ Ein Post-Implementation-Review kann unterschiedliche Konsequenzen haben. ${ }^{337}$ So führte z. B. die Überprüfung von IFRS 13 Bemessung des beizulegenden Zeitwerts zu keiner Standardänderung oder -ergänzung, ${ }^{338}$ hingegen bei IFRS 3 zu zwei Folgeprojekten, die nicht nur einzelne Klarstellungen und zusätzliche Anwendungsleitlinien in IFRS 3, sondern auch ein Forschungsprojekt zur Überarbeitung von IAS 36 Wertminderung von Vermögenswerten umfassten $^{339}$. Während der Post-Implementation-Review einer uneinheitlichen Standardanwendung nach Standardveröffentlichung entgegenwirken soll, kann er gleichzeitig zu einer anwendungsorientierten Konkretisierung der Standards führen.

\subsection{Standarderläuterung durch das IFRS Interpretations Committee}

Die Implementierung und konsistente Anwendung der Standards wird neben dem IASB unterstützend vom IFRS Interpretations Committee sichergestellt. ${ }^{340}$ Anwendungsprobleme können von Rechnungslegungsadressaten an das IFRS

\footnotetext{
${ }^{333}$ Vgl. Due Process Handbook, Rz. 6.48 f.

${ }^{334}$ Vgl. Due Process Handbook, Rz. 6.48-6.54.

335 Vgl. Moldovan (2014), S. 114.

${ }^{336} \mathrm{Vgl}$. Due Process Handbook, Rz. 6.56 f.; zum Einbezug der Forschung Ewert/Wagenhofer (2012a), S. 281-284.

${ }^{337}$ Vgl. Due Process Handbook, Rz. 6.58.

338 Vgl. IASB (2018b), S. 3.

${ }^{339}$ Vgl. IASB (2015), S. 5 f.; zum Überblick über die finalen Änderungen in IFRS 3 Meyer (2019), S. 1388-1390.

${ }^{340}$ Vgl. IFRS Constitution, Rz. 42.
} 
Interpretations Committee herangetragen werden, das ergänzend zur Standardsetzung des IASB sog. IFRIC-Interpretationen entwickelt, Änderungsvorschläge an das IASB weiterleitet oder bestehende Regelungen im Rahmen von Agenda-Entscheidungen noch einmal erläutert. ${ }^{341}$ IFRIC-Interpretationen sind als Bestandteil der IFRS-Standards vom IASB zu bestätigen und werden dem gleichen Standardsetzungsverfahren folgend unter Veröffentlichung zu kommentierender Standardentwürfe entwickelt. ${ }^{342}$ Das IFRS Interpretations Committee bezweckt entsprechend der angestrebten prinzipienorientierten Standardsetzung keine detaillierten Regelungen in Form umfangreicher Standardanpassungen, sondern eine standarderläuternde Interpretation bestehender Vorschriften. ${ }^{343}$ Die Entwicklung einer IFRIC-Interpretation erfolgt dabei nicht im Fall von Ermessensfragen, sondern nur bei übergeordneten Auslegungsproblemen. ${ }^{344}$

Für die Aufnahme eines Projekts auf die Agenda bedarf es eines Anliegens, das einen weiten Adressatenkreis betrifft und für das die anzuwendenden Regelungen keine hinreichende Basis für eine angemessene Bilanzierung liefern, wodurch unterschiedliche Anwendungspraktiken bestehen, das jedoch innerhalb der Standardgrenzen sowie mithilfe des Rahmenkonzepts gelöst werden kann und das unter Kostengesichtspunkten gerechtfertigt ist. ${ }^{345}$ Bspw. wurde IAS 12 Ertragsteuern aufgrund wesentlicher Unterschiede in den Bilanzierungsansätzen zur Berücksichtigung von Ertragssteuerrisiken durch IFRIC 23 Unsicherheit bezüglich der ertragssteuerrechtlichen Behandlung ergänzt, ${ }^{346}$ der die vorherigen Möglichkeiten der einzelfallspezifischen Ergebnissteuerung begrenzt. ${ }^{347}$ Obwohl die angestrebte Prinzipienorientierung eine branchen- und transaktionsunabhängige Standardsetzung verlangen würde, sind einzelne IFRIC-Interpretationen als industriespezifische Erläuterungen zu charakterisieren. ${ }^{348}$ So wurde z. B. die vor IFRS 15 bestehende Problematik der Standardanwendung zur Umsatzerfassung aus Werklieferungsverträgen anstatt durch eine für Mehrkomponentengeschäfte allgemeingültige Standardanpassung oder -ergänzung mithilfe des speziell für

\footnotetext{
${ }^{341}$ Vgl. Due Process Handbook, Rz. 5.15 f., 7.1, 8.2.

342 Vgl. Due Process Handbook, Rz. 7.2-7.4.

343 Vgl. Due Process Handbook, Rz. 5.13, 7.6.

344 Vgl. Bradbury (2007), S. 117.

345 Vgl. Due Process Handbook, Rz. 5.16.

${ }^{346}$ Vgl. IFRS Interpretations Committee (2014), Agenda Paper 5A.

347 Vgl. Kovermann/Velte (2017), S. 408 f.

348 Vgl. etwa IFRIC 1 Änderungen bestehender Rückstellungen für Entsorgungs-, Wiederherstellungs- und ähnliche Verpflichtungen, IFRIC 5 Rechte auf Anteile an Fonds für Entsorgung, Rekultivierung und Umweltsanierung, IFRIC 20; ferner zur Häufigkeit bestimmter Themen Bradbury (2007), S. $114 \mathrm{f}$.
} 
die Errichtung von Immobilien geltenden IFRIC 15 Verträge über die Errichtung von Immobilien gelöst. ${ }^{349}$ Obwohl die Erstellung von IFRIC-Interpretationen Anwendungsprobleme beseitigen soll, kann ihr Umfang und Detailgrad im Einzelfall bestehende Bilanzierungspraktiken ändern, ${ }^{350}$ wie IFRIC 23 , oder einzelne Auslegungsfragen nicht umfassen, wie IFRIC 15, weshalb weiterhin unterschiedliche Anwendungspraktiken möglich sind, die wiederum einen neuen Standardsetzungsprozess bedingen können.

Sofern das IFRS Interpretations Committee bei der Lösung von Anwendungsfragen im Rahmen der Standardauslegung keinen Konsens findet oder diese nur durch Änderungen des Standards adressiert werden können, sind die Anwendungsfragen an das IASB weiterzuleiten. ${ }^{351}$ Dies kann sowohl einzelne Nachbesserungsprojekte, wie etwa die geringfügige Anpassung von IAS 16 Sachanlagen zur Berücksichtigung von Einnahmen vor der beabsichtigen Nutzung von Sachanlagen, ${ }^{352}$ oder auch gänzlich neue Standardsetzungsprojekte bewirken, wie z. B. das bislang inaktive Forschungsprojekt zur Bilanzierung von Verbindlichkeiten aus variablen und bedingten Gegenleistungen ${ }^{353}$. In den meisten Fällen führen die an das IFRS Interpretations Committee herangetragenen Anfragen jedoch weder zu einer IFRIC-Interpretation noch zu einer Standardanpassung durch das IASB ${ }^{354}$ sondern zu sog. Agenda Decisions, in denen das IFRS Interpretations Committee seine Entscheidung zur Nichtaufnahme eines Standardsetzungsprojekts begründet. ${ }^{355}$

Die Veröffentlichung von Agenda-Entscheidungen erfolgt in der Regel bei Anwendungsfragen zur Auslegung - umfasst mitunter indes auch Ermessensfragen - komplexer Standards, ${ }^{356}$ für die eine adäquate Bilanzierungslösung jedoch anhand bestehender Standards oder mithilfe des Rahmenkonzepts gefunden werden kann, weshalb die Agenda-Entscheidungen im Wesentlichen eine erklärende Funktion erfüllen. ${ }^{357}$ So werden z. B. in den bislang zu IFRS 15 veröffentlichten Agenda-Entscheidungen Anwendungsfragen zur Umsatzerfassung

\footnotetext{
${ }^{349}$ Vgl. Brune (2016), § 9, Rz. 111-114.

350 Vgl. Camfferman/Zeff (2015), S. 394 f.

351 Vgl. Due Process Handbook, Rz. 5.18.

352 Vgl. IFRS Interpretations Committee (2016), Agenda Paper 2; Technical Staff (2016c), Agenda Paper 12B, Rz. $1 \mathrm{f}$.

${ }^{353}$ Vgl. IFRS Interpretations Committee (2016), Agenda Paper 8; IASB (2016b), S. 3.

354 Vgl. Bradbury (2007), S. $111 \mathrm{f}$.

${ }^{355}$ Vgl. Due Process Handbook, Rz. 5.19 i. V. m. 8.2 f.

356 Vgl. Bradbury (2007), S. 114-117.

${ }^{357}$ Vgl. Due Process Handbook, Rz. 8.3.
} 
aus Immobilienverträgen, zur Identifizierung bestimmter Leistungsverpflichtungen bei Wertpapiergeschäften sowie zur Erfassung der in Zusammenhang mit der Erstellung eines Guts anfallenden Kosten ausführlich anhand der Regelungen des IFRS 15 erläutert. ${ }^{358}$ Agenda-Entscheidungen sind daher mit den einen IFRSStandard ergänzenden Beispielen vergleichbar ${ }^{359}$ und fördern die einheitliche Anwendung der Regelungsvorschriften ${ }^{360}$. Obwohl sie keine standardvergleichbare Verbindlichkeit besitzen, sollten sie jedoch im Rahmen der Standardanwendung Berücksichtigung finden und können demzufolge auch eine Änderung der Bilanzierungspraxis bei Unternehmen erfordern; ${ }^{361}$ sie entfalten damit de facto eine standardkonkretisierende Wirkung. ${ }^{362}$ Die Legitimationsfunktion des Due Process wird durch diese Verlagerung des Standardsetzungsprozesses gleichwohl unterminiert. So sind besonders mögliche Auswirkungen von Agenda-Entscheidungen auf einen standardimmanenten Ermessensspielraum vor dem Hintergrund, dass sich die Kompetenz des IFRS Interpretations Committee formal auf Auslegungs-, nicht jedoch Ermessensfragen erstreckt, fraglich.

Die Betrachtung der einzelnen Standardsetzungsphasen ,veranschaulich[t], dass in der Standardsetzung zur internationalen Rechnungslegung nur der Wandel beständig bleibt.“363 Der Standardsetzungsprozess ist aufgrund des rekursiven Zusammenhangs zwischen Standardsetzung und Standardanwendung dabei durch eine stetige Kompromissfindung und damit Wertungsentscheidung zwischen Theorie und Praxis geprägt. ${ }^{364}$ Die Partizipationsmöglichkeiten der am Standardsetzungsprozess Beteiligten sind daher im Folgenden als eine das Standardsetzungsergebnis erklärende Komponente zu betrachten.

\footnotetext{
358 Vgl. IFRS Interpretations Committee (2018), Agenda Paper 2C, 2D, 2E; IFRS Interpretations Committee (2019a), Agenda Paper 3; IFRS Interpretations Committee (2019b), Agenda Paper 10.

${ }^{359}$ Vgl. Bradbury (2007), S. 120.

${ }^{360}$ Vgl. Due Process Handbook, Rz. 8.3.

${ }^{361}$ Vgl. Due Process Handbook, Rz. 8.4-8.6.

${ }^{362}$ Vgl. Camfferman/Zeff (2015), S. 394 f.; zur Bestätigung der Relevanz durch Bezugnahme der ESMA-Prüfungsschwerpunkte ESMA (2018), S. 2-5.

363 Wüstemann (2014), S. 1.

${ }^{364}$ Vgl. Beresford (1988), S. 6 f.; Fogarty (1992), S. 337.
} 


\subsection{Kommunikations- und Feedbackwege während des Standardsetzungsprozesses}

\subsection{Partizipationsmöglichkeiten für Interessengruppen}

2.2.2.1.3.1.1 Anwendungsorientierte Empfehlungen durch Mitarbeit in Beratungsgruppen

Die Kommunikation zwischen dem IASB und den Interessengruppen kann auf direkte oder indirekte Weise in unterschiedlichen Phasen des Standardsetzungsprozesses erfolgen, wobei Interessengruppen unterschiedliche Möglichkeiten $\mathrm{zu}$ verschiedenen Zeitpunkten auf Basis ihrer individuellen Kosten-NutzenAbwägung wahrnehmen können. ${ }^{365}$ Besonders Unternehmen als Jahresabschlussersteller und Anwender der Rechnungslegungsvorschriften haben im Fall negativer ökonomischer Konsequenzen eines Standardentwurfs aufgrund ihres positiven Kosten-Nutzen-Verhältnisses einen hohen Anreiz, sich am Standardsetzungsprozess zu beteiligen. ${ }^{366}$ Während Stellungnahmen, öffentliche Anhörungen und Gesprächsrunden oder die Teilnahme an Feldstudien, informellen Treffen sowie Beratungsgremien eine direkte Einflussnahme ermöglichen, kann durch Kommentierungen in den Medien, Anfragen an die EU, die European Financial Reporting Advisory Group (EFRAG), nationale Standardsetzer sowie Unternehmens- und Wirtschaftsverbände oder die Förderung von Forschungsarbeiten über Dritte indirekt Einfluss genommen werden. ${ }^{367}$ Einzelne Partizipationsmöglichkeiten sind als (quasi) verpflichtende Verfahrensanforderungen vom IASB bzw. der IFRS Foundation vorgegeben; ${ }^{368}$ sie bilden die Voraussetzung für eine Kompromissfindung, indem mögliche Kritikpunkte eines Standards durch den Einbezug der Interessengruppen - oder zumindest den Verweis hierauf - adressiert werden können. ${ }^{369}$

Ein gezielter Dialog wird bei umfangreichen Standardsetzungsprojekten normalerweise durch die Schaffung spezieller Beratungsgruppen angestrebt, die für die Beurteilung der Anwendbarkeit von Standards zusätzliche praktische Erfahrungen und Fachwissen einbringen. ${ }^{370}$ Die Zusammensetzung derartiger Expertengruppen richtet sich in Abhängigkeit des jeweiligen Standards nach den

\footnotetext{
365 Vgl. Sutton (1984), S. 89-92.

366 Vgl. Sutton (1984), S. 85 f.; Francis (1987), S. 38-54.

367 Vgl. Georgiou (2004), S. 222; Georgiou (2010), S. 106.

368 Vgl. Due Process Handbook, Rz. 3.59.

369 Vgl. Fogarty (1994), S. 220 f.

${ }^{370}$ Vgl. Due Process Handbook, Rz. 3.44 f.; Maier (2008), S. 209.
} 
benötigten, themenrelevanten Fachkenntnissen und kann sich während eines Standardsetzungsprojekts entsprechend ändern. ${ }^{371}$ Die IFRS Foundation kann auch projektunabhängig einzelne adressatenspezifische Beratungsgremien, bspw. aus Investoren oder Jahresabschlusserstellern, gründen, die bei regelmäßigen Treffen Hinweise sowie Empfehlungen zu verschiedenen Themen erarbeiten. ${ }^{372}$ Durch dieses „network of organizations surrounding the IASB“373 können interessengruppenspezifische Empfehlungen zu einem Standardsetzungsprojekt bereits während der Standardentwicklung einfließen. So setzt sich das Capital Markets Advisory Committee aus Analysten zusammen, um nutzerorientierte Einschätzungen zu liefern, das Global Preparers Forum hingegen aus Unternehmensvertretern, um bilanzierungspraktische Rückmeldungen zu geben. ${ }^{374}$ Durch die Teilnahme an beratenden Gremien können Interessengruppen anwendungsorientierte Empfehlungen aussprechen, die vom IASB im Rahmen der Standardsetzung berücksichtigt werden und das Standardsetzungsergebnis beeinflussen können. ${ }^{375}$

Im Unterschied zu Post-Implementation-Reviews, die erst nach erfolgter Standardanwendung und damit auf Basis einer vorläufig herausgebildeten Bilanzierungspraxis vom IASB durchgeführt werden, können potenzielle Implementierungsfragen bei neueren Standardsetzungsprojekten von Interessengruppen zudem bereits unmittelbar nach Standardveröffentlichung an sog. Transition Resource Groups (TRG) herangetragen werden ${ }^{376}$. Die TRG setzen sich standardspezifisch aus Unternehmensvertretern und Vertretern von Wirtschaftsprüfungsgesellschaften zusammen, die die in den Stellungnahmen aufgeworfenen Anwendungsprobleme diskutieren. ${ }^{377}$ Während bei IFRS 9 Anwendungsfragen durch die bestehenden Regelungen erläutert wurden, veranlassten die Ergebnisse der TRGTreffen zu IFRS $15^{378}$ und IFRS $17^{379}$ das IASB zu einer Standardklarstellung im Rahmen einer nachgelagerten Standardentwurfs- und Konsultationsphase.

\footnotetext{
371 Vgl. Due Process Handbook, Rz. 3.60.

372 Vgl. Due Process Handbook, Rz. 3.61.

373 Camfferman/Zeff (2018), S. 304.

${ }^{374}$ Vgl. Technical Staff (2018d), Agenda Paper 1D, Rz. 17, 22; The Capital Markets Advisory Committee (2019), S. 1; Global Preparers Forum (2017), Part A.

${ }^{375} \mathrm{Vgl}$. etwa zur Berücksichtigung erlangter Erkenntnisse insgesamt Technical Staff (2019a), Agenda Paper 1A sowie Technical Staff (2019b), Agenda Paper AP1B; zur Wahrnehmung von Unternehmen, dass die Teilnahme an Beratungsgremien effektiv ist, Georgiou (2010), S. 110.

${ }^{376}$ Vgl. Technical Staff (2018d), Agenda Paper 1D, Rz. 51-54, 62-68.

377 Vgl. etwa Technical Staff (2018d), Agenda Paper 1D, Rz. 51.

378 Vgl. ED Clarifications (2015), Introduction.

${ }^{379}$ Vgl. IASB (2019), Rz. BC4-BC8.
} 
Obwohl die TRG formal keine Standardsetzungskompetenz besitzen, können die veröffentlichten und ausführlich diskutierten TRG-Agenda-Paper indes nicht nur im Rahmen der Erkenntniserlangung des IASB in die Standardsetzung einfließen, sondern auch - insbesondere bei vom IASB nicht aufgegriffenen Themen - faktisch bei der Standardanwendung Berücksichtigung finden. ${ }^{380}$

\subsection{2 Implementierungsorientierte Rückmeldungen durch Teilnahme an Feldstudien}

Neben der Einflussnahme als Mitglied einer Beratungsgruppe können Interessengruppen dem IASB durch die Teilnahme an Feldstudien anwendungsrelevantes Feedback geben. ${ }^{381} \mathrm{Im}$ Rahmen persönlicher Einzeltreffen oder Interviews sowie Workshops und Fallstudien mit verschiedenen Interessengruppen sollen das Verständnis sowie die möglichen Auswirkungen neuer Regelungsvorschriften untersucht und Erkenntnisse über ihre Anwendbarkeit, besonders auch in Bezug auf bestimmte Branchenpraktiken erlangt werden. ${ }^{382}$ Die Durchführung von Feldstudien dient dabei nicht der Kommunikation persönlicher Auffassungen zu den vorgeschlagenen Standardentwürfen, sondern der Überprüfung der Umsetzbarkeit der Regelungsvorschläge im Sinne eines Implementierungstests $^{383}$ oder der Identifizierung unterschiedlicher Bilanzierungspraktiken ${ }^{384}$, und soll folglich die Praktikabilität der Regelungsvorschriften sicherstellen ${ }^{385}$. So wurde bspw. bei der Entwicklung des Rahmenkonzepts aufgrund der vom IASB erachteten geringen Anwendungsrelevanz auf einen Feldtest verzichtet. ${ }^{386}$ Trotz kontroverser Anwendungsprobleme, wie etwa im Fall des Rahmenkonzeptprojekts, ${ }^{387}$ folgt die Entscheidung zur Durchführung eines Feldtests demnach einer Kosten-Nutzen-Abwägung ${ }^{388}$. Obwohl das IASB bei einzelnen Standardsetzungsprojekten, wie bspw. bei IFRS 17 , mehrere Feldstudien vornimmt, ${ }^{389}$ werden

\footnotetext{
380 Vgl. etwa Technical Staff (2018d), Agenda Paper 1D, Rz. 63, 68.

381 Vgl. Due Process Handbook, Rz. 3.70.

$382 \mathrm{Vgl}$. Due Process Handbook, Rz. $3.71 \mathrm{f}$.

${ }^{383}$ Vgl. etwa IASB (2007), Rz. 17 f.; IASB (2009), S. 2 f.

${ }^{384}$ Vgl. Effects Analysis Consultative Group (2014), S. 45.

385 Vgl. IFRS Constitution, Rz. 36 (k).

386 Vgl. Technical Staff (2014b), Agenda Paper 10 H, Rz. 38.

387 Vgl. zur Kritik der Stellungnehmer Erb/Pelger (2015a), S. 28-32.

388 Vgl. Maier (2008), S. 210 f.

${ }^{389} \mathrm{Vgl}$. IFRS 17.BC6 (b).
} 
sie bei vielen Projekten aufgrund des relativ hohen Kosten- und Ressourceneinsatzes häufig von der Eigeninitiative der Interessengruppen angetrieben ${ }^{390}$ und indessen von der EFRAG durchgeführt, wie bspw. bei IFRS 9 und IFRS $15^{391}$. Die im Rahmen von Feldstudien erlangten Erkenntnisse sind zusammen mit den erhaltenen Stellungnahmen vom IASB bei der Standardentwicklung zu berücksichtigen ${ }^{392}$ und können eine Anpassung der Regelungsvorschläge oder Ergänzung von Anwendungsleitlinien begründen ${ }^{393}$. Sie liefern folglich - insbesondere für Unternehmen - eine wichtige Basis für eine praxisorientierte Einflussnahme.

\subsection{3 Individuelle Standardbeurteilung durch Stellungnahmen}

Unter den Partizipationsmöglichkeiten kommt den Stellungnahmen als verpflichtender Verfahrensbestandteil eine entscheidende Bedeutung $\mathrm{zu}$, da durch sie individuelle Ansichten und Vorschläge zu einem Standardsetzungsprojekt an das IASB adressiert werden können. ${ }^{394}$ Durch Stellungnahmen kann sowohl die Zustimmung als auch die Ablehnung eines Regelungsvorschlags zum Ausdruck gebracht werden. ${ }^{395}$ Während der Anreiz zur Kommentierung bei Interessengruppen, die nicht von einem Standardentwurf betroffen sind oder diesem (gänzlich) zustimmen, eher gering ist, ist besonders bei ablehnenden Positionen eine verstärkte Teilnahme zu erwarten. ${ }^{396}$ Bei Standardentwürfen, die bspw. von der bestehenden Bilanzierungspraxis abweichen, ist demzufolge mit einem erhöhten Widerstand zu rechnen. ${ }^{397}$ Als Grundlage der Stellungnahmen dient der jeweilige Standardentwurf; die Antworten sind jedoch frei gestaltbar, weshalb die Stellungnehmer nicht auf die Wahl vorgegebener Lösungsansätze beschränkt sind, sondern alternative Regelungsvorschriften oder Anpassungen vorschlagen können. ${ }^{398}$ Die Standardbeurteilung erfolgt innerhalb der Grenzen des Vorverständnisses bestehender Regelungen ${ }^{399}$ sowie etablierter Rechnungslegungspraktiken und wird insbesondere im Fall der Ablehnung einzelner Regelungsvorschläge regelmäßig

\footnotetext{
${ }^{390}$ Vgl. Maier (2008), S. 210 f.

391 Vgl. etwa EFRAG (2014), S. 2.

392 Vgl. Due Process Handbook, Rz. 3.74.

${ }^{393}$ Vgl. etwa Technical Staff (2012b), Agenda Paper 16E, S. 12-18.

394 Vgl. Due Process Handbook, Rz. 3.67.

${ }^{395} \mathrm{Vgl}$. Francis (1987), S. 37 f.

396 Vgl. Schalow (1995), S. 30, 36; Durocher u. a. (2007), S. 31.

${ }^{397}$ Vgl. Elbannan/McKinley (2006), S. $601 \mathrm{f}$.

${ }^{398}$ Vgl. Sutton (1984), S. 84; Tietz-Weber (2006), S. 56.

${ }^{399}$ Vgl. Young (1996), S. 491.
} 
durch eine - zum Teil eigennutzorientierte - Begründung der jeweiligen Position in Form ökonomischer und/oder konzeptioneller Argumente gestützt ${ }^{400}$. Die Überzeugungskraft einer Stellungnahme wird nicht nur durch die Wahl der Argumente, sondern auch durch die Verwendung rhetorischer Mittel bedingt, da bspw. mithilfe von Analogien und Metaphern Sachverhalte und Positionen anschaulich, mithin greifbarer, vermittelt werden können. ${ }^{401}$

Die Stellungnahmen sind vom IASB im Standardsetzungsprozess zu berücksichtigen, wobei ihr Einfluss von den vorgebrachten Erklärungen und Nachweisen abhängt. ${ }^{402}$ Eine Analyse der Stellungnehmer und ihrer Herkunft sowie interessengruppenspezifischer Auffassungen können dem IASB zudem Hinweise für die Durchführung weiterer standardsetzungsrelevanter Maßnahmen liefern. ${ }^{403}$ Die Berücksichtigung von Stellungnahmen erhöht dabei nicht nur die Akzeptanz und folglich die Legitimation des IASB, sondern wirkt sich auch auf den Inhalt und damit die Qualität finaler Standards aus. ${ }^{404}$ Im Gegensatz zur Teilnahme durch Beratungsgruppen und Feldstudien, in denen das IASB durch anwendungsrelevante Hinweise in erster Linie zusätzliche Erkenntnisse erlangt, entspricht die Teilnahme durch Stellungnahmen dem primären Aushandlungsprozess der Standards, da die unterschiedlichen Positionen und ihre Erwiderungen in die zu erarbeitenden Rechnungslegungsvorschriften einfließen ${ }^{405}$.

Die im Fall des Dissens zwischen den Ansichten der Interessengruppen sowie der Zielsetzung des IASB notwendige Kompromissfindung erfordert dabei eine Abwägung der ,,conceptual purity and practical considerations“. ${ }^{406}$ Inwieweit die in den Stellungnahmen hervorgebrachten Positionen und verwendeten Argumente in diesen ,judgemental process“ ${ }^{\star 407}$ einfließen, ist im Einzelfall unterschiedlich. So wurde bspw. beim Rahmenkonzeptprojekt mit dem Austausch der Anforderung der Verlässlichkeit durch die Anforderung der glaubwürdigen Darstellung entgegen den in den Stellungnahmen adressierten Bedenken

\footnotetext{
${ }^{400}$ Vgl. Tutticci u. a. (1994), S. 94-98; Jupe (2000), S. 346-354; Giner/Arce (2012), S. 670674.

${ }^{401}$ Vgl. Cornelissen u. a. (2011), S. 1705 f.; Stenka (2013), S. 7 f.; Okamoto (2017), S. 136. 402 Vgl. Due Process Handbook, Rz. 3.69; zum Einfluss der Qualität der Informationen Hansen (2011), S. 73.

403 Vgl. Due Process Handbook, Rz. 3.69.

${ }^{404}$ Vgl. Durocher/Fortin (2011), S. 29; Larson/Herz (2013), S. 103; kritisch zum Einfluss von Stellungnahmen auf die inhaltliche Ausgestaltung von Standards Botzem (2010), S. 143147.

405 Vgl. Hussein/Ketz (1991), S. 76; Fogarty (1992), S. 337.

406 Vgl. Beresford (1988), S. 5-7 (auch Zitat, S. 6).

${ }^{407}$ Beresford (1988), S. 5; vgl. auch Teixeira (2014), S. 5.
} 
die konzeptionelle Stärkung der Entscheidungsnützlichkeit verfolgt. ${ }^{408}$ Während einzelne Regelungen des IFRS 15 - bspw. die Abkehr von dem anfänglich verfolgten Fair-Value-Ansatz ${ }^{409}$ oder der bilanztheoretisch konsequenten Umsetzung des Kontroll-Kriteriums ${ }^{410}$ - hingegen von einem Zugeständnis zugunsten praktischer Erwägungen gekennzeichnet sind, verfolgte das IASB bei der Ausgestaltung der Regelungen zur Aufteilung von Leistungsverpflichtungen und des Transaktionspreises anstatt einer praktikablen Ausnahme - etwa in Form der Contingent Revenue Cash-Cap ${ }^{411}$ - wiederum eine konzeptionell stringente Lösung. Die Abwägung zwischen konzeptionellen und praktischen Überlegungen verläuft nicht zuletzt in den konfligierenden Grenzen eines politischen und technischen Entscheidungsprozesses ${ }^{412}$, der je nach Standardsetzungsprojekt durch unterschiedliche Faktoren, bspw. auch dem Einfluss des Staff oder der Board-Zusammensetzung, ${ }^{413}$ bedingt wird. Vor diesem Hintergrund sind die vom IASB zu einem Standardsetzungsprojekt durchgeführten Effektanalysen sowie die Begründungen in den einen Standard ergänzenden Basis for Conclusions wesentlich für die diskursive Auseinandersetzung im Standardsetzungsprozess und folglich der Legitimation entwickelter Standards.

\subsection{Rechtfertigung entwickelter Rechnungslegungsvorschriften durch Kosten-Nutzen-Verhältnis berücksichtigende Effektanalysen}

Das IASB führt im Rahmen jedes Standardsetzungsverfahrens Effektanalysen durch, die der Einschätzung der Kosteneffizienz von Regelungsvorschlägen dienen. $^{414}$ Dabei hat das IASB in den einzelnen Standardsetzungsphasen zu beurteilen, wie sich die Jahresabschlüsse aufgrund neuer bzw. überarbeiteter Vorschriften möglicherweise ändern, ob diese Veränderungen die Qualität der Jahresabschlüsse erhöhen und ob die Änderungen gerechtfertigt sind, sowie seine Ansichten zu begründen. ${ }^{415}$ Gleichzeitig ermöglichen Effektanalysen dem IASB

\footnotetext{
408 Vgl. Erb/Pelger (2015a), S. 26-34.

${ }^{409}$ Vgl. Wüstemann/Kierzek (2005b), S. 430-432; Hommel u. a. (2009), S. 377; Schipper u. a. (2009), S. $61 \mathrm{f}$.

${ }^{410}$ Vgl. Wüstemann/Wüstemann (2014), S. 933 f.; Wagenhofer (2014), S. 367 f.

${ }^{411} \mathrm{Vgl}$. Wüstemann (2018), S. $10 \mathrm{f}$.

412 Vgl. Young (2014), S. 718-743.

${ }^{413}$ Vgl. Morley (2016), S. 231-249; Pelger (2016), S. 55-69.

414 Vgl. Due Process Handbook, Rz. 3.76 f.

415 Vgl. Due Process Handbook, Rz. 3.76 i. V m. 3.79.
} 
etwaigen nicht substantiierten Behauptungen von Interessengruppen entgegenzuwirken und diese begründet zu entkräften. ${ }^{416}$ Die Umschreibung als Effektanalyse umfasst im Sinne eines breiten Kosten-Nutzen-Verständnisses die Berücksichtigung ökonomischer Konsequenzen. ${ }^{417}$ Für die Analyse werden daher neben den erhaltenen Stellungnahmen die mit betroffenen Akteuren durchgeführten Feldstudien und Konsultationen einbezogen. ${ }^{418}$

Während die Ergebnisse der Effektanalysen bislang als Teil der einen Standard ergänzenden Basis for Conclusions veröffentlicht wurden, erfolgte mit IFRS 16 und IFRS 17 die separate Veröffentlichung der Ergebnisse. ${ }^{419}$ Im Gegensatz zu der häufig komprimierten Auseinandersetzung mit den Auswirkungen neuer Standards innerhalb der Basis for Conclusions beinhalten die separat veröffentlichten Analysen eine detaillierte und ausführliche Beurteilung, die auch industriespezifische und quantitative Einschätzungen umfasst ${ }^{420}$. Die separate Veröffentlichung der Effektanalysen ist auf die Empfehlung der für die Stärkung und Entwicklung eines einheitlichen Vorgehens von Effektanalysen gegründeten Beratungsgruppe zurückzuführen ${ }^{421}$ und dient durch die umfangreichere Informationsbereitstellung wiederum der Standardakzeptanz.

Die Ermittlung der Kosten und Nutzen bzw. die Bewertung der einzelnen Effekte sind trotz der Transparenzbestrebungen und des Einbezugs verschiedener Informationsquellen aufgrund der inhärenten Beurteilungsschwierigkeiten jedoch ermessensbehaftet und werden unvermeidlich durch die politisch motivierte Komponente des Entscheidungsprozesses bedingt. ${ }^{422}$ Die effizienzbasierte Analyse entspricht insofern vielmehr einer konsensorientierten Abwägung. ${ }^{423}$ Entsprechend der auf Rahmenkonzeptebene als Kostenrestriktion festgelegten Verfahrensanforderung ${ }^{424}$ spiegelt die Effektanalyse mithin die vorgenommene Abwägung konzeptioneller Ansprüche in Form der qualitativen Anforderungen und praktischer Erwägungen in Form ökonomischer und sozialer Folgen

\footnotetext{
${ }^{416}$ Vgl. Teixeira (2014), S. 6 f.

${ }^{417}$ Vgl. Due Process Handbook, Rz. 3.80; ausführlich zu unterschiedlichen Effekten sowie einer differenzierten Würdigung Haller u. a. (2012), S. 115-120.

418 Vgl. Due Process Handbook, Rz. 3.76; zum Nutzen und zur Forderung des expliziten Einbezugs akademischer Forschung Trombetta u. a. (2012), S. 138-143; Haller u. a. (2012), S. 122-124.

419 Vgl. Due Process Handbook, Rz. 3.81.

${ }^{420}$ Vgl. etwa IASB (2016c), S. $16 \mathrm{f}$.

${ }^{421}$ Vgl. Effects Analysis Consultative Group (2014), S. 32.

${ }^{422}$ Vgl. Haller u. a. (2012), S. 124; Madsen (2013), S. 81-83.

${ }^{423}$ Vgl. Madsen (2013), S. 82 f., bezugnehmend auf McLeay u. a. (2000), S. 79.

${ }^{424}$ Vgl. zur Kostenrestriktion auf Rahmenkonzeptebene Abschnitt 2.1.2.2.2.3.
} 
wider. Änderungen der bestehenden Bilanzierungspraxis können dabei konzeptionell mit dem Argument des überwiegenden Nutzens gerechtfertigt werden. ${ }^{425}$ So ist bspw. die Abschaffung der Contingent Revenue Cash-Cap trotz kostenintensiver Änderungen für einzelne Industrien mit dem mutmaßlich größeren Nutzen aus der sachgerechten Abbildung der wirtschaftlichen Substanz der Transaktionen begründbar. ${ }^{426}$ Ein weniger kostenintensiver oder die bestehende Bilanzierungspraxis erhaltender Regelungsvorschlag kann indes durch den Verweis auf die kosteneffizientere Lösung gerechtfertigt werden, wie z. B. die zugunsten der erleichterten Anwendbarkeit vom einzelvertragsbasierten Ansatz abweichende Portfolio-Option des IFRS 15. ${ }^{427}$ Inwiefern eine Entscheidung kosten- oder nutzenorientiert bzw. praxisgerecht oder konzeptionell gerechtfertigt wird, ist demnach subjektiv geprägt, ${ }^{428}$ und kann zu einer Erhöhung der Standardkomplexität führen, sofern ein Kompromiss z. B. durch (inkonsistente) Ausnahmeregelungen oder zusätzliche Anwendungsleitlinien geschaffen wird $^{429}$. Obwohl dies der angestrebten konsistenten Standardsetzung entgegensteht, sind derartige Kompromisse aufgrund des standardsetzungsimmanenten Legitimationserfordernisses jedoch nicht unwahrscheinlich.

\subsection{Rechtfertigung entwickelter Rechnungslegungsvorschriften in der Basis for Conclusions}

Neben Effektanalysen, die Einblicke in die Gesamtbeurteilung eines Standards vermitteln, legen die Begründungen zur Ausgestaltung einzelner Vorschriften auf Ebene der jeweiligen regelungszugehörigen Erläuterung innerhalb der Basis for Conclusions den einzelfallspezifischen Entscheidungsprozess offen. In den Basis for Conclusions erläutert das IASB die im Standardsetzungsprozess getroffenen Entscheidungen und reagiert auf die eingegangenen Stellungnahmen, ${ }^{430}$ weshalb sie als Erwägungsgründe gleichsam eine Rechtfertigungsgrundlage darstellen. Da die Akzeptanz neuer bzw. überarbeiteter Standards wesentlich von der Fähigkeit des IASB abhängt, die Adressaten von der Richtigkeit der entwickelten Rechnungslegungsvorschriften zu überzeugen, ${ }^{431}$ erfüllen die Erläuterungen innerhalb der Basis for Conclusions eine legitimierende Funktion. Das Rahmenkonzept

\footnotetext{
425 Vgl. Fogarty (1994), S. 218.

${ }^{426}$ Vgl. Teixeira (2014), S. 7, bezugnehmend auf Brown (2013), S. 857.

$427 \mathrm{Vgl}$. IFRS 15.BC488; ebenso IFRS 16.BC43.

428 Vgl. IFRS 15.BC455.

${ }^{429}$ Vgl. Herz (2006), S. 21.

${ }^{430}$ Vgl. Due Process Handbook, Rz. 3.82.

${ }^{431}$ Vgl. Young (1995), S. 173-175.
} 
kann im „decision-making or reasoning process[.]“ als Bezugspunkt bzw. Würdigungsmaßstab genutzt werden. ${ }^{432}$ Die Entwicklung eines „guten Standards“ verlangt neben einer konzeptionellen Fundierung jedoch auch die Berücksichtigung ökonomischer Erwägungen, weshalb die Argumente der Realitätsabbildung und der Entscheidungsnützlichkeit sowie der Konsistenz und Praktikabilität miteinander verknüpft werden, um den Entscheidungsprozess überzeugend zu kommunizieren und dadurch die Akzeptanz der Regelungsvorschriften zu erhöhen. ${ }^{43}$ Die vom IASB genutzte Rhetorik, wie etwa die Verwendung von Methapern in Form der Personifizierung von Standarddokumenten oder der metonymische Verweis auf das öffentliche Interesse oder die typisierten Nutzer, dient dabei zur Überzeugung einer objektiv richtigen Lösung. ${ }^{434}$ Durch die Bezugnahme auf die eingegangenen Stellungnahmen können hierdurch nicht nur die von Interessengruppen angebrachten Kritikpunkte vom IASB gezielt adressiert werden, sondern auch Widerstand sowie Änderungsforderungen seitens der Stellungnehmer unterbunden werden. ${ }^{435}$

Sofern ein Board-Mitglied der Veröffentlichung eines Standardentwurfs oder finalen Standards nicht zustimmt, sind die Gründe für die abweichende Meinung ebenfalls in der Basis for Conclusions darzulegen. ${ }^{436}$ Obwohl der Umstand, dass nicht einzelnen Regelungen widersprochen werden kann, sondern nur eine Ablehnung des gesamten Standards bzw. Standardentwurfs möglich ist, eine relativ hohe Hürde darstellt und der Veröffentlichung im Fall der mehrheitlichen Zustimmung nicht entgegensteht, ${ }^{437}$ weist die fehlende Kompromissfähigkeit zwischen den Board-Mitgliedern auf mögliche Defizite des Standards hin. So lehnte ein Board-Mitglied bspw. die Veröffentlichung des Re-Exposure Drafts von IFRS 15 mit der Begründung der inkonsistenten Ausgestaltung der Regelungen - etwa in Bezug auf die Konkretisierung des zeitraumbezogenen Kontrollübergangs - ab. ${ }^{438}$ Auch bei der Überarbeitung von IAS 39 Finanzinstrumente: Ansatz und Bewertung respektive der Entwicklung von IFRS 9 äußerten mehrere Board-Mitglieder aufgrund der im Vergleich zum Status quo fehlenden Verbesserung sowie der

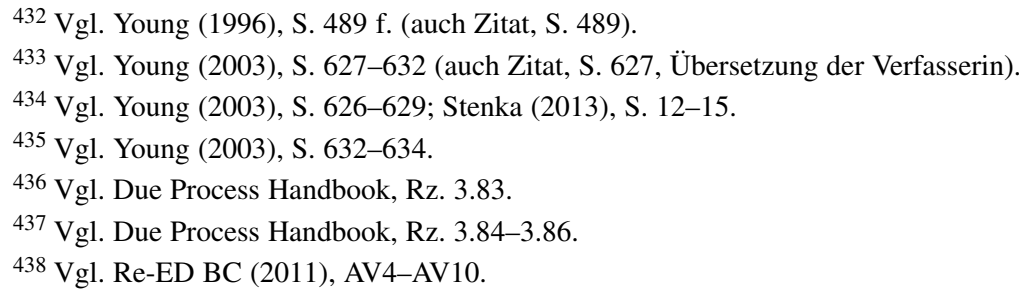


Defizite in der Anwendbarkeit und der Entscheidungsnützlichkeit vorgeschlagener Regelungen ihre Ablehnung. ${ }^{439}$

Die fehlende Zustimmung einzelner Board-Mitglieder verdeutlicht die Schwierigkeiten der Kompromissbildung bereits auf Ebene des Standardsetzers. Der Entscheidungsprozess wird zudem durch die unterschiedlichen Meinungen der Interessengruppen, besonders wenn sie die eigene Position durch Verweis auf die abweichende Meinung eines Board-Mitglieds sowie die angeführten Argumente stützen, weiter erschwert. Die Entwicklung neuer oder wesentliche Überarbeitung bestehender Standards mit dem Ziel der Verbesserung der Rechnungslegung 440 ist aufgrund des häufig bestehenden Zielkonflikts zwischen einer konzeptionellen Neuausrichtung und einer praxisgerechten Fortentwicklung daher eher durch „evolution rather than revolution“ gekennzeichnet. ${ }^{441}$ Dieser graduelle Wan$\mathrm{del}^{442}$ in der Standardsetzung wird wiederum durch das rekursive Verhältnis zur Standardanwendung bedingt.

\subsubsection{Weiterentwicklung von Rechnungslegungsvorschriften im Rahmen der Standardanwendung}

\subsection{Normenhierarchie}

Das System der IFRS besteht aus den vom IASB erlassenen Standards ${ }^{443}$ sowie dem integralen Anhang in Form von Anwendungsleitlinien, der sog. Application Guidance, (IAS 8.9) und wird durch die standardzugehörigen, jedoch nicht verbindlichen Basis for Conclusions, Illustrative Examples und Practice Statements sowie dem Rahmenkonzept, das ebenfalls keinen IFRS-Standard darstellt, ergänzt. ${ }^{444}$ Der unterschiedliche Verbindlichkeitsgrad lässt sich mit der angestrebten Prinzipienorientierung rechtfertigen, wird jedoch durch die praktische Anwendungsrelevanz der zusätzlichen Veröffentlichungen konterkariert. Innerhalb der EU ist die verpflichtende Anwendung der IFRS für kapitalmarktorientierte Unternehmen zur Erstellung konsolidierter Jahresabschlüsse an

\footnotetext{
${ }^{439}$ Vgl. IFRS 9.BC Dissenting Opinions, S. 340-354; grundlegend zur Analyse verwendeter Argumente bei der Standardablehnung Bradbury/Harrison (2015), S. 367-373; Bradbury/Harrison (2012), S. 8-14.

440 Vgl. Due Process Handbook, Rz. 3.85.

${ }^{441}$ Vgl. Macve (1997), S. xxii f. (auch Zitat); ebenso Walton (2018), S. 197.

442 Vgl. Young (1994), S. 84 f.

${ }^{443} \mathrm{Zu}$ den IFRS gehören neben den IAS- und IFRS-Standards auch die SIC- und IFRICInterpretationen.

444 Vgl. Wawrzinek/Lübbig (2016), § 2, Rz. 1; Driesch (2016a), § 1, Rz. 46.
} 
die Übernahme der Standards in europäisches Recht gebunden. ${ }^{445}$ Im Rahmen des Endorsement-Verfahrens ${ }^{446}$ werden - dem Verbindlichkeitscharakter des IASB folgend - nur die einzelnen IFRS-Standards sowie die zugehörigen Anwendungsleitlinien geprüft und durch die EU verpflichtend normiert; sowohl die standardergänzenden Dokumente als auch (bislang) das Rahmenkonzept $^{447}$ sind nicht Bestandteil des Übernahmeverfahrens und erlangen somit keine Rechtsverbindlichkeit. ${ }^{448}$ Aufgrund ihrer erläuternden Funktion entfalten sie als Erkenntnisquellen für Unternehmen bei der Standardanwendung, insbesondere bei Ermessensspielräumen, Auslegungsfragen und Regelungslücken, jedoch eine faktische Verbindlichkeit. ${ }^{449}$ Neue IFRS-Standards, für die noch kein Endorsement erfolgte, können ebenfalls im Rahmen der RegelungslückenschlieBung Anwendung finden, sofern sie nicht mit bereits in EU-Recht übernommene Standards kollidieren. ${ }^{450}$

Das Konglomerat aus verbindlichen Rechnungslegungsstandards, deren Regelungsvorschriften möglichst prinzipienorientiert festgelegt werden, und unverbindlichen Zusatzdokumenten, die eher regelorientierte Einzelsachverhalte umfassen, verhindert weder Auslegungsspielräume noch Regelungslücken. ${ }^{451}$ So sind die von Unternehmen abzubildenden Geschäftsvorfälle und Transaktionen häufig durch eine hohe Komplexität gekennzeichnet und werden durch verändertere Umweltzustände sowie neue Sachverhalte bedingt. ${ }^{452}$ Während die in den IFRS gewährten Ermessensspielräume sowie die Vorschriften zur SchlieBung von Regelungslücken die Abbildung von Sachverhaltsänderungen in der Standardanwendung ermöglichen, erfordern neue Geschäftsmodelle aufgrund der mit der Zielsetzung der Informationsvermittlung angestrebten sachgerechten Abbildung der Vermögens-, Finanz- und Ertragslage gleichzeitig die Überarbeitung bzw. Entwicklung neuer Regelungen durch das IASB im Rahmen der Standardsetzung ${ }^{453}$. „Die Evolution eines Rechnungslegungsystems wird

\footnotetext{
${ }^{445}$ Vgl. IAS-Verordnung, Artikel 4.

${ }^{446}$ Vgl. zum Ablauf sowie historischer und aktueller Entwicklungen van Mourik/Walton (2018), S. 3-25.

447 Vgl. zur Diskussion der Übernahme Merkt (2014), S. 497-499.

${ }^{448}$ Vgl. Jödicke (2008), S. 26-28; Wawrzinek/Lübbig (2016), § 2, Rz. 1 f.

${ }^{449}$ Vgl. Wawrzinek/Lübbig (2016), § 2, Rz. 2.

${ }^{450}$ Vgl. zur Diskussion unterschiedlicher Konstellationen Pellens u. a. (2007), S. 2504-2507; Hennrichs (2014), Rz. 60-64.

${ }^{451}$ Vgl. SEC (2003), Abschnitt I. D; van den Eynden (2010), S. 9 f.

452 Vgl. Jödicke (2008), S. 7.

${ }^{453}$ Vgl. Young (1996), S. 492; van den Eynden (2010), S. 10.
} 
[demzufolge] wesentlich durch den Umgang mit neuen Sachverhalten [...] determiniert." ${ }^{\text {454 }}$ Beispielhaft hierfür sind etwa auch die entwickelten Regelungen zur Bilanzierung von Mehrkomponentengeschäften in IFRS $15 .{ }^{455}$

\subsection{Normauslegung und Ermessensausübung}

Unternehmen haben als Abschlussersteller die zu bilanzierenden Sachverhalte durch Anwendung der entsprechenden IFRS-Regelungen abzubilden (IAS 8.7). Da vom IASB weder eine Normierung jeglicher Anwendungsfälle in Form von Einzelnormen angestrebt wird noch eine derartige Festlegung aufgrund der Vielseitigkeit und Innovation wirtschaftlicher Transaktionen abschließend möglich ist, verlangt die Standardanwendung regelmäßig die Bedeutungserschließung einer Norm mittels Auslegung. ${ }^{456}$ Von der zur Sachverhaltsabbildung im Einzelfall erforderlichen Ermessensausübung sowie der Auslegung abstrakt oder vage formulierter Regelungen sind gezielte Sachverhaltsgestaltungen, die auf ein Umgehen von Regelungen abzielen, abzugrenzen. ${ }^{457}$ Für die Normauslegung ist aufgrund der Übernahme der IFRS in europäisches Recht und der damit formal letztlich verbundenen Entscheidungskompetenz des EuGH bzw. nationaler Gerichte der in der juristischen Hermeneutik verwendete Auslegungskanon heranzuziehen. ${ }^{458}$ Ausgehend vom Wortlaut der Regelung sollte der Entstehungsprozess und die systematische Einbettung der Vorschrift sowie insbesondere der Telos der Norm berücksichtigt werden. ${ }^{45}$ Zwar sind das Rahmenkonzept, die Basis for Conclusions und die Illustrative Examples wie auch die im Rahmen des Standardsetzungsprozesses veröffentlichten, aber überworfenen Dokumente sowie die Agenda-Entscheidungen des IFRS Interpretations Committee als unverbindliche Standarddokumente kein verbindliches Gemeinschaftsrecht, sie stellen jedoch Erkenntnisquellen bei der Normauslegung dar. ${ }^{460}$

\footnotetext{
454 Jödicke (2008), S. 7.

${ }^{455}$ Vgl. Wagenhofer (2014), S. 365; Teixeira (2014), S. 7.

456 Vgl. Jödicke (2008), S. 17-23.

${ }^{457}$ Vgl. Tanski (2006), S. 31-36; zur Abgrenzung des Earnings Managements Healy/Wahlen (1999), S. 368 f.; Ewert/Wagenhofer (2012b), S. 1-3.

458 Vgl. Schön (2004), S. 764-766; Hennrichs (2014), Rz. 65.

${ }^{459}$ Vgl. Nerlich (2007), S. 276-313; Hennrichs (2014), Rz. 66-72.

${ }^{460}$ Vgl. Küting/Ranker (2004), S. 2511; Hennrichs (2014), Rz. 70-75.
} 
Unbestimmte Rechtsbegriffe sowie Vorschriften, die eine Schätzung oder Wahrscheinlichkeits- und Wesentlichkeitsbeurteilungen erfordern, sind im Rahmen der Standardanwendung von Unternehmen für den jeweiligen Bilanzierungssachverhalt zu konkretisieren (IAS 1.122 f.; IAS 8.10). ${ }^{461}$ Während der in IAS 1 und IAS 8 grundlegende Verweis auf die Ermessensausübung des Managements auch eine subjektive Auslegung nahelegt, ${ }^{462}$ ergibt sich aufgrund des Rechtsnormcharakters der IFRS aus den bisherigen Beschlüssen des Oberlandesgerichts (OLG) Frankfurt zur Überprüfung der IFRS-Anwendung im Rahmen von Enforcement-Verfahren für deutsche Unternehmen vielmehr die Notwendigkeit einer objektiven Auslegung ${ }^{463}$. Demnach wird die Fehlerhaftigkeit einer Auslegung des Unternehmens durch die Bundesanstalt für Finanzen (BaFin) ${ }^{464}$ selbst bei Vertretbarkeit subjektiver Auslegungen der Unternehmen auf Basis der „objektiv richtige[n] Rechtslage“ bestimmt. ${ }^{465}$ Dieser objektive Fehlerbegriff „setzt eine im Widerspruch zu den objektiven Verhältnissen stehende unrichtige Darstellung voraus“, ${ }^{466}$ weshalb „,bei der Ermittlung des Rechnungslegungsverstoßes zu prüfen ist, ob eine bestimmte Art und Weise der Bilanzierung noch mit den Vorgaben der IFRS in Einklang steht“467. Nicht die vom bilanzierenden Unternehmen vertretene Rechtsauffassung, sondern die von BaFin bzw. Gerichten ermittelte objektive Rechtslage ist für die Auslegung maßgeblich; ${ }^{468}$ bei Ermessensfragen sollten hingegen weiterhin auch vertretbare Entscheidungen - sofern sachgerecht - nicht zur Beanstandung führen. ${ }^{469} \mathrm{Da}$ die durch das deutsche Rechtsverständnis geprägte Unterscheidung zwischen Normauslegung und Ermessensausübung in den IFRS nicht in dieser Eindeutigkeit besteht

461 Vgl. Küting (2011), S. 2091-2093; zur Übersicht unbestimmter Regelungen in den IFRS Tanski (2006), S. 55-140; Scheffler (2006), S. 4-6.

462 Vgl. Lüdenbach u. a. (2016), § 24, Rz. 39; Driesch (2016b), § 45, Rz. 41; Beyhs u. a. (2012), S. 656.

${ }^{463}$ Vgl. OLG (2016), Rz. 51-53; OLG (2019), Rz. 88-94.

464 Vgl. grundlegend zum deutschen Enforcement-Modell Bockmann (2012), S. 145-183.

465 Vgl. OLG (2019), Rz. 89-100 (auch Zitat, Rz. 94); OLG (2016), Rz. 51-53.

466 Vgl. Lüdenbach u. a. (2016), § 24, Rz. 39 (auch Zitat).

${ }^{467}$ OLG (2019), Rz. 88.

${ }^{468}$ Vgl. OLG (2019), Rz. 100 f.; Böcking u. a. (2019), S. 2645.

${ }^{469}$ Vgl. bereits Scheffler (2006), S. 7; Küting u. a. (2007), S. 7; zum subjektiven und objektiven Fehlerbegriff im deutschen Bilanzrecht sowie zur Unterscheidung zwischen Auslegung und Ermessen Weber-Grellet (2013), S. 730-733; Schulze-Osterloh (2013), 1132 f.; zur Abgrenzung vertretbarer Auslegungen anhand unterschiedlicher Rechtsebenen Lüdenbach/Freiberg (2019a), S. 2309. 
bzw. häufig fließend ist, ${ }^{470}$ sollten Unternehmen bei der Konkretisierung unbestimmter Regelungsvorschriften auch veröffentlichte Fehlerfeststellungen sowie die ergangene Rechtsprechung berücksichtigen, ihre Übertragbarkeit auf die zu würdigenden Sachverhalte jedoch im Einzelfall überprüfen.

Beispielhaft kann die für die Vertragszusammenfassung gemäß IFRS 15.17 auszulegende Bedingung des „geringe[n] Zeitabstand[s]“ zwischen dem Abschluss zweier oder mehrerer Verträge betrachtet werden. ${ }^{471}$ Die Erwägungsgründe in der Basis for Conclusions deuten darauf hin, dass mit der Bedingung der Zeitnähe eine Restriktion übermäßiger Vertragszusammenfassungen bezweckt wurde, die Beurteilung mithin zunächst formal durch den zeitlichen Vertragsabschluss und nicht primär anhand wirtschaftlicher Abhängigkeiten zu bestimmen ist. ${ }^{472}$ Gleichzeitig begründet das IASB jedoch, dass die Anforderung der zeitlichen Nähe der glaubwürdigen Darstellung der Unternehmensperformance dienen soll und als solche eine Ermessensausübung erfordert ${ }^{473}$, weshalb sie anstatt einer strikten Begrenzung der wirtschaftlichen Betrachtungsweise vielmehr einen Entscheidungsrahmen vorgibt. Auch eine weite Auslegung ${ }^{474}$ des Zeitraums innerhalb derer die Verträge geschlossen werden, scheint daher in Abhängigkeit von der rechtlichen Würdigung des jeweiligen Sachverhalts begründbar und im Einzelfall etwa für geschlossene Verlustverträge sowie Rahmenwerklieferungsverträge erforderlich. ${ }^{475}$ Inwieweit derartige Regelungen als Auslegungsfragen im Rahmen eines Enforcement-Verfahrens überprüft werden und hierfür jeweils eine einzige objektiv richtige Auffassung auch sachverhaltsunabhängig festgestellt werden kann oder diese vielmehr als Ermessensentscheidungen einzustufen sind, ist daher fraglich. ${ }^{476}$ Mit IAS 1 und IAS 8 scheint indes gerade durch das Erfordernis der Ermessensausübung im Rahmen der Standardanwendung ein flexibles und transaktionsunabhängiges Rechnungslegungssystem vom IASB

${ }^{470}$ Vgl. Lüdenbach/Freiberg (2019a), S. 2306 f.; Scheffler (2006), S. 2 f.; zur kritischen Diskussion des objektiven Fehlerbegriffs im Rahmen des Enforcements Hennrichs (2009), S. $1447 \mathrm{f}$.

471 Vgl. Wüstemann u. a. (2017a), Rz. 36 f.

${ }^{472} \mathrm{Vgl}$. IFRS 15.BC75.

${ }^{473} \mathrm{Vgl}$. IFRS 15.BC75.

${ }^{474}$ Vgl. Lüdenbach u. a. (2016), § 25, Rz. 42.

475 Vgl. Grote u. a. (2014), S. 409; Schurbohm-Ebneth/Viemann (2015a), S. 182; Konold/Müller (2015), S. 6; Wüstemann u. a. (2017b), S. 1196 f.

${ }^{476}$ Vgl. etwa IFRS 15.BC75, in dem das IASB klarstellt, ,that an entity should apply judgement". 
beabsichtigt, das auch eine - sofern nicht im Widerspruch zu geltenden Vorschriften - uneinheitliche, jedoch den Einzelfall berücksichtigende und insofern sachgerechte Konkretisierung einzelner Regelungen ermöglicht. ${ }^{477}$

\subsection{Schließung von Regelungslücken}

\subsection{Analoge Anwendung ähnlicher Standards}

Sofern ein Sachverhalt unter keiner bestehenden Regelung subsumiert werden kann, obliegt es dem Unternehmen eine geeignete Bilanzierungslösung zu entwickeln (IAS 8.10). Regelungslücken können entweder (un)bewusst vom Standardsetzer gesetzt werden, etwa weil für die Lösung eines bestimmten Bilanzierungsproblems kein Konsens erreicht wird, oder nachträglich aufgrund von technischen oder wirtschaftlichen Änderungen, wie bspw. durch die Einführung aktienorientierter Entlohnungsformen, entstehen. ${ }^{478}$ Auch die Bilanzierung von Versicherungsverträgen war nicht nur bis zur Entwicklung von IFRS 4 Versicherungsverträge ein „regelungsfreie[r] Raum“4779, sondern wird bis zur Übernahme von IFRS 17 durch den Verweis auf die Anwendung nationaler Bilanzierungspraktiken bestimmt ${ }^{480}$. Versicherungsunternehmen haben anstelle der für die Schließung von Regelungslücken in IAS 8.10-12 festgelegten Vorgehensweise nationale Regelungsvorschriften anzuwenden (IFRS 4.13) und diese zu modifizieren, sofern eine Änderung die Relevanz oder Vergleichbarkeit erhöht (IFRS 4.22), mithin ,,...] diese Regelungslücken durch ein von ihnen selbst zu entwickelndes Regelwerk zu schließen. “481 Ein derartiger Ausgestaltungsspielraum ermöglicht Unternehmen dabei von Normanwendern zu Normentwicklern zu werden. ${ }^{482}$

Im Regelfall hat das Management jedoch bei der Schließung von Regelungslücken die vom IASB vorgegebene Hierarchie an Erkenntnisquellen einzuhalten und eine Rechnungslegungsmethode anzuwenden, die eine relevante und verlässliche Informationsvermittlung gewährleistet (IAS 8.10 f.). Unternehmen haben dabei primär zu prüfen, ob durch die Anwendung ähnlicher und verwandter IFRSStandards eine sachgerechte Bilanzierungslösung gefunden werden kann (IAS 8.11 (a)). Mit der analogen Anwendung vergleichbarer Standards wird zunächst

\footnotetext{
477 Vgl. im Ergebnis ebenso Lüdenbach/Freiberg (2019a), S. 2309; Lüdenbach/Freiberg (2019b), S. 2648.

478 Vgl. Jödicke (2008), S. 13-15, 111-116.

${ }^{479}$ Hommel (2003), S. 2114.

$480 \mathrm{Vgl}$. IFRS 17.IN4.

${ }^{481}$ Vgl. Bacher (2006), S. 127-129 (auch Zitat, S. 129).

482 Vgl. zum ,corporate norm-entrepreneurship“ Wolf u. a. (2010), S. 19; Okamoto (2017), S. $134 \mathrm{f}$.
} 
eine systemimmanente Lösung durch das IASB angestrebt, ${ }^{483}$ wonach kodifizierte Regelungen auf Anwendungsfälle ausgedehnt werden, die den geregelten Fällen ähneln ${ }^{484}$. So sollte etwa die Einrechnung nicht abzugsfähiger Vorsteuerbeträge bei der Bewertung des Nutzungsrechts gemäß IFRS 16 aufgrund fehlender Definition des Anschaffungskostenbegriffs unter Zuhilfenahme der Regelungen des IAS 16 gelöst werden können. ${ }^{485}$ Die durch Analogieschluss angewendeten Regelungen dürfen jedoch nicht mit einschlägigen Regelungsvorschriften in Konflikt stehen. ${ }^{486}$ So scheiterte z. B. eine analoge Anwendung von IAS 11, IFRIC 4 Feststellung, ob eine Vereinbarung ein Leasingverhältnis enthält, IFRIC 13 Kundenbindungsprogramme und IFRIC 15 zur Schließung der vor Inkrafttreten von IFRS 15 bestehenden faktischen Regelungslücke in IAS $18^{487}$ zur Bilanzierung von Mehrkomponentengeschäften aufgrund bestehender Widersprüche zu einzelnen Vorschriften des IAS $18 .^{488}$

\subsection{Anwendung des Rahmenkonzepts}

Sofern ein Analogieschluss nicht möglich ist, sind Regelungslücken unter Zuhilfenahme der Definitions-, Ansatz- und Bewertungsvorschriften für Bilanz- und Ertragsposten des Rahmenkonzepts zu schließen (IAS 8.11 (b)). Obwohl dieser verbindliche Einbezug gemäß IAS 8 aufgrund des fehlenden Endorsements des Rahmenkonzepts eine „Legitimationslücke“ darstellt, ${ }^{489}$ hat der Verweis in materieller Hinsicht wenig Auswirkungen. So sind die Vorschriften des Rahmenkonzepts überwiegend ,allgemeiner Natur und interpretationsbedürftig, folglich flexibel.“490 Die „abstrakten“ Ertragsvereinnahmungskriterien des Rahmenkonzepts ermöglichten daher auch keine Lösung der vor IFRS 15 bestehenden Regelungslücke in IAS 18 zur Bilanzierung von Mehrkomponentengeschäften. ${ }^{491}$ Durch die Abschaffung der noch im alten Rahmenkonzept bestehenden Ansatzkriterien des wahrscheinlichen Nutzenzuflusses sowie der verlässlichen

483 Vgl. Ranker (2006), S. 73.

${ }^{484}$ Vgl. Ruhnke/Nerlich (2004), S. 393 f.; grundlegend zur analogen Rechtsanwendung Winnefeld (2015), Rz. 78.

485 Vgl. Ebeling/Fröbel (2019), S. 157 f.; zu weiteren Beispielen Jödicke (2008), S. 58-62.

${ }^{486}$ Vgl. zu Analogieverboten Jödicke (2008), S. 62-64; zum Verhältnis von Analogieschluss und Ausnahmeregelungen Schild (2018), S. $1138 \mathrm{f}$.

487 Vgl. Wüstemann/Kierzek (2007c), S. 903; Sessar (2007), S. 266-269.

488 Vgl. Fürwentsches (2010), S. 58-62; Sessar (2007), S. 274-283.

${ }^{489} \mathrm{Vgl}$. Hoffmann/Detzen (2012), S. 54 f. (auch Zitat, S. 54).

490 Vgl. Jödicke (2008), S. 72 (auch Zitat); Ranker (2006), S. 74.

${ }^{491}$ Vgl. Grau (2002), S. 59-63 (auch Zitat, S. 60); Wüstemann/Kierzek (2005a), S. 80 f.; Sessar (2007), S. 274. 
Bewertbarkeit ${ }^{492}$ wird die Konkretisierungsfunktion des Rahmenkonzepts weiter beschränkt. Auch die unterschiedlichen Bewertungsmaßstäbe eröffnen im Einzelfall eine Bandbreite möglicher Bilanzierungsalternativen. ${ }^{493}$ Das Rahmenkonzept ist für die Deduktion einer einheitlichen Bilanzierungslösung im Rahmen der Lückenschließung folglich nur bedingt geeignet. ${ }^{494}$

\subsection{Anwendung anderer Rechnungslegungsstandards und anerkannter Branchenpraktiken}

Sofern weder die Regelungen ähnlicher Standards noch das Rahmenkonzept eine Bilanzierungslösung für Regelungslücken vorgeben, kann das Management gemäß IAS 8.12 die auf einem vergleichbaren konzeptionellen Rahmenkonzept basierenden Rechnungslegungsvorschriften anderer Standardsetzer sowie sonstige Verlautbarungen und anerkannte Branchenpraktiken anwenden, sofern sie nicht mit bestehenden IFRS-Vorschriften oder dem Rahmenkonzept des IASB in Konflikt stehen. ${ }^{495}$ Der systemfremde Analogieschluss ermöglicht dabei eine Anwendung nationaler Rechnungslegungsstandards, wie etwa den US-GAAP oder der Vorschriften des Deutschen Rechnungslegungs Standards Committee (DRSC), als auch nicht rechtskräftiger Quellen, wie bspw. den Empfehlungen des Instituts der Wirtschaftsprüfer (IDW). ${ }^{496}$ Während die US-GAAP aufgrund ihrer kapitalmarktorientierten Ausrichtung und dem zumindest in Teilen übereinstimmenden Rahmenkonzept vorwiegend geeignet erscheinen, sollte der analogen Anwendung des Handelsgesetzbuchs (HGB) bzw. der Grundsätze ordnungsgemäßer Buchführung $(\mathrm{GoB})$ indes die im Vergleich zu den IFRS unterschiedliche Zwecksetzung und die damit einhergehende abweichende konzeptionelle Basis in den meisten Fällen entgegenstehen. ${ }^{497}$ Obwohl diese ,rechtsvergleichende Komponente" für die Schließung von Regelungslücken nicht verbindlich ist und in jedem Einzelfall geprüft werden muss, erlangt sie in der Bilanzierungspraxis eine faktische Anwendungsrelevanz. ${ }^{498}$ So erfolgte bspw. die Umsatzerfassung

492 Vgl. zu den Implikationen der überarbeiteten Definitions-, Ansatz- und Bewertungsvorschriften Erb/Pelger (2018a), S. 328-330.

${ }^{493}$ Vgl. Jödicke (2008), S. 70-72; Barker (2006), S. 173 f.

${ }^{494}$ Vgl. Ranker (2006), S. 73 f.; Jödicke (2008), S. 69-74; Najderek (2009), S. 119 f.; Schober (2020), S. 24-27.

495 Vgl. zur Notwendigkeit der EU-rechtskonformen Lückenschließung anhand der Endorsement-Kriterien Najderek (2009), S. 120 f.

496 Vgl. Wawrzinek/Lübbig (2016), § 2, Rz. 115.

${ }^{497}$ Vgl. zur kritischen Würdigung der Anwendbarkeit unterschiedlicher Standards Jödicke (2008), S. 75-85.

498 Vgl. Nerlich (2007), S. 225 f. (auch Zitat, S. 226). 
aus Mehrkomponentengeschäften vor IFRS 15 aufgrund des möglichen Rückgriffs auf die US-GAAP-Regelungen durch Anwendung des EITF 00-21 Revenue Arrangements with Multiple Deliverables. ${ }^{499}$

Neben vergleichbaren Rechnungslegungsstandards können auch anerkannte Branchenpraktiken zur Schließung von Regelungslücken angewendet werden (IAS 8.12). Dieser praktikabilitätsorientierte Verweis entspricht einer pragmatischen Anknüpfung, die konzeptionell nur mit einer Erhöhung der - wenn auch industriespezifischen - Vergleichbarkeit gerechtfertigt werden kann, und sollte vor allem branchenspezifische Regelungslücken, wie etwa in der Rohstoff- und Energie- oder der Versicherungsbranche, betreffen. ${ }^{500}$ Unternehmen können in diesem Fall eine für die sachverhaltsspezifischen Besonderheiten der abzubildenden Geschäftsvorfälle sachgerechte Bilanzierungslösung entwickeln, die sich branchenweit ausdehnen kann. Inwieweit jedoch durch anerkannte - möglicherweise abweichende - Branchenpraktiken eine konsistente Bilanzierung erreicht wird, ist hingegen fraglich. ${ }^{501}$

Die Betrachtung der Standardanwendung zeigt, dass Unternehmen sowohl bei der Schließung von Regelungslücken als auch bei der Ermessensausübung in unterschiedlichem Ausmaß als „Normentwickler“ agieren. Sofern ein Rechnungslegungsstandard überarbeitet oder ein neuer Standard entwickelt wird und dabei bestehende (praxisgerechte) Ermessensspielräume reduziert oder bislang existierende Regelungslücken geschlossen werden, für die sich jedoch eine Bilanzierungspraxis etabliert hat, ist daher mit einem erhöhten Widerstand bzw. einer Einflussnahme zugunsten der Beibehaltung oder der Übernahme der entwickelten Bilanzierungspraxis zu rechnen. ${ }^{502}$ Gleichzeitig fördert das notwendige Legitimationserfordernis im Rahmen der Standardsetzung kompromissfähige Lösungen, die durch unbestimmte Rechtsbegriffe und Ermessensspielräume zur Anwendung auf eine Vielzahl unterschiedlicher Sachverhalte geeignet sind. Eine Konkretisierung im Rahmen der Anwendung ist vor diesem Hintergrund erforderlich. Die Standardanwendung stellt somit sowohl Antrieb als auch Hindernis für die Standardsetzung und folglich den Wandel eines Rechnungslegungssystems dar. Vor diesem Hintergrund werden im Folgenden die Standarddokumente zu IFRS 15

\footnotetext{
${ }^{499}$ Vgl. Wüstemann/Kierzek (2007c), S. 904 f.; Fürwentsches (2010), S. 69-75, 134-136.

500 Vgl. Jödicke (2008), S. 85-87.

501 Vgl. Jödicke (2008), S. 85-87.

502 Vgl. ausführlich zu möglichen Gründen der Ablehnung Elbannan/McKinley (2006),

S. 608-616; ferner Jorissen u. a. (2012), S. 712-714.
} 
sowie die zugehörigen Stellungnahmen von Unternehmen zu relevanten Regelungen zur Bilanzierung von Mehrkomponentengeschäften mithilfe qualitativinhaltsanalytischer Verfahren untersucht, um Erkenntnisse darüber zu erlangen, wie Unternehmen als Standardanwender und das IASB als Standardsetzer auf die Entwicklung von Rechnungslegungsvorschriften einwirken.

Open Access Dieses Kapitel wird unter der Creative Commons Namensnennung 4.0 International Lizenz (http://creativecommons.org/licenses/by/4.0/deed.de) veröffentlicht, welche die Nutzung, Vervielfältigung, Bearbeitung, Verbreitung und Wiedergabe in jeglichem Medium und Format erlaubt, sofern Sie den/die ursprünglichen Autor(en) und die Quelle ordnungsgemäß nennen, einen Link zur Creative Commons Lizenz beifügen und angeben, ob Änderungen vorgenommen wurden.

Die in diesem Kapitel enthaltenen Bilder und sonstiges Drittmaterial unterliegen ebenfalls der genannten Creative Commons Lizenz, sofern sich aus der Abbildungslegende nichts anderes ergibt. Sofern das betreffende Material nicht unter der genannten Creative Commons Lizenz steht und die betreffende Handlung nicht nach gesetzlichen Vorschriften erlaubt ist, ist für die oben aufgeführten Weiterverwendungen des Materials die Einwilligung des jeweiligen Rechteinhabers einzuholen.

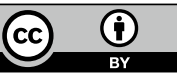

